\title{
REVUE CRITIQUE
}

\section{LES COPÉPODES PARASITES DE MOLLUSQUES}

\author{
Par Théodore MONOD et Robert-Ph. DOLLFUS
}

\section{SOMMAIRE}

1. Introduction . . . . . . . . . 129

2. Nomenclator generum..................................... 130

3. Nomenclator specierum ou liste des espèces de Copépodes p.rasites ou commensaux de Mollusques................ 133

4. Nomenclator hospilum ou liste systématique des Mollusques hébergeant des Copépodes parasites ou commensaux....... 180

5. Addendum. . . . . . . . . . . . . . . . . . . . . . . . . . . 198

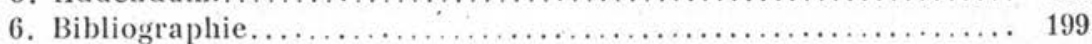

\section{INTRODUCTION}

Le présent travail a été entrepris il y a plusieurs années et annoncé déjà à deux reprises (Dollfus, 1927, p. 121 ; Monod, 1928, p. 18).

Nous souhaitons qu'il puisse compléter sur certains points la Revue des Copépodes de mollusques récemment publiée par P. Pelseneer (1928) et contribuer à faciliter l'étude de parasites qui mériteraient d'attirer davantage l'attention des zoologistes.

Bien entendu, nous sommes les premiers à reconnaître combien est essentiellement arbitraire un isolement systématique artificiel des Copépodes parasites de Mollusques, considérés à part, sans tenir compte des espèces parasites de Colentérés, d'Echinodermes, de Géphyriens et d'Ascidies. Une étude comparative détaillée, intéressant l'ensemble des Copépodes parasites d'invertébrés, conduirait sans aucun doute à des conclusions taxonomiques importantes, en particulier à la mise en place, dans des familles connues, de bien. des genres de position actuellement tout à fait incertaine.

Pour beaucoup d'espèces, les renseignements que nous donnons ici sont très fragmentaires; nous n'avons pu nous procurer le matériel nécessaire pour les compléter.

Annales de Parasitologie, T. $X, \mathrm{~N}^{\circ} 2,-1^{\text {er }}$ mars 1932, p. 129-204. 
Nous devons remercier : M. le professeur Paul Pelseneer, qui a eu l'obligeance de nous renseigner sur la provenance de plusieurs des espèces signalées par lui ; M. Jean Risbec, qui a bien voulu nous envoyer de Nouvelle-Calédonie quelques-unes de ses récentes captures ; Mme Pruvot-Fol, qui s'est aimablement dessaisie en notre faveur d'exemplaires de Splanchnotrophus.

\section{NOMENCLATOR GENERUM}

1. Acontiophorus Brady $(1880$, p. 23,69$)$. [nom. nov. pro Solenostoma Brady et Robertson 1873]. Génotype par préséance : Acontiophorus scutatus (Brady et Robertson 1873).

2. Ameristocheres Pelseneer (1928, p. 41). Monotypique : Ameristocheres inermis Pelseneer 1928.

3. Anchicaligus Stebbing (1900, pp. 667-668). Monotypique : Anchicaligus nautili (Willey 1896), Stebbing 1900.

4. Anthessius Della Valle (1880, p. 122). Monotypique : Anthessius solecurti Della Valle 1880 [err. typ. = solenocurti].

5. Artotrogus Boeck (1859, p. 2). Monotypique : Artotrogus orbicularis Boeck 1859.

6. Boeckia Brady (1872, p. 6). Monotypique : Boeckia arenicola Brady 1872.

7. Bomolachus (Alder et Hancock, 1855, p. 27, légende des figures 8-9, pl. XLV). [Err. typ. = Bomolochus, nec Bomolochus von Nordmann 1832. (Génotype : Bomolochus belones Bürmeister 1833, par désignation) $]=$ Splanchnotrophus Hancock et Norman (1863, p. 50).

8. Briarella Bergh (1876, p. 408). Monotypique : Briarella microcephala Bergh 1876.

9. Caligus O.-F. Müller (1785, pp. 128-129). Génotype par préséance : Caligus curtus O.-F. Müller 1785.

10. Cerastocheres Monod (novum genus, vide infra). Monotypique : Cerastocheres trochicola Monod eod. loc.

11. Cholidya Farran (1914, p. 473). Monotypique : Cholidya polypi Farran 1914.

12. Chondrocarpus Bassett-Smith (1903, pp. 104-105). Génotype par préséance : Chondrocarpus reticulosus Bassett-Smith 1903.

12 bis. Conchoceres Pelseneer (1928, p. 35). Err. typ. $=$ Conchocheres G.-O. Sars 1918.

(1) Nous meationnons les orthographes défectueuses par lapsus ou erreurs typographiques. 
13. Conchocheres G.-O. Sars (1918, p. 201). Monotypique : Conchocheres malleolatus G.-O. Sars 1918.

14. “ Doricola ") in Nils $\mathrm{Hj}$. Odhner (1922, p. 33). Lapsus pro Doridicola Leydig 1853.

15. Doridicola Leydig (1853, p. 377). Monotypique : Doridicola agilis Leydig 1853.

16. Eolidicola M. Sars (1862, p. 138). Monotypique : Eolidicola tenax M. Sars 1872 .

17. Ergasilus (Alder et Hancock 1855, p. 27) [nec Ergasilus von Nordmann 1832] = Lichomolgus Thorell 1860. [Ergasilus Boas (1886, p. 34) est un Copépode indéterminé, probablement un Lichomolgidé].

18. Hermannella auctorum (Normann et A. Scott 1906. p. 199 ; G.-O. Sars 1918, p. 171. etc.). Err. typ. = Herrmannella Canu 1891.

19. Herrmannella Canu (1891, pp. 479-480). Monotypique : Herrmannella rostrata Canu 1891. - D'autres auteurs, et Canu lui-même (1894, p. 3) ont écrit Hermannella, graphie erronée, le genre était dédié à G. Herrmann (Canu 1891, p. 479).

20. Ischnurella Pelseneer (1928, p. 40). Monotypique : Ischnurella barneæ Pelseneer 1928.

21. Ismaïla Bergh (1867, p. 118). Monotypique : Ismaïla monstrosa Bergh 1867.

22. Ismaila Bergh (1867, p. 97 ; 1868 , pp. 133,137 ; 1879, p. 558; 1898 , p. 506). Bien que la graphie Ismaila ait une priorité de page sur Ismaïla (1867, pp. 97 et 118), il est manifeste que Bergh a voulu écrire Ismaïla en 1867, quoiqu'il ait souvent répété Ismaila par la suite.

23. Ismailia Bergh (1879, p. 558). Err. typ. = Ismaïla Bergh 1867.

24. Ismalia Bassett-Smith (1899, p. 495). Err. typ. = Ismaïla Bergh 1867.

25. Leptinogaster Pelseneer (1928, p. 37). Monotypique : Leptinogaster pholadis Pelseneer 1928.

26. Lichomolgus Thorell (1860, pp. 64-69). Génotype par préséance : Lichomolgus albens Thorell 1860.

27. Linckiomolgus Stebbing (1900, p. 664). Monotypique : Linckiomolgus cæruleus Stebbing 1900.

28. Lomaniticola Hecht (1896, p. 87). Err. typ. = Lomanoticola T. et A. Scott 1895 .

29. Lomanoticola T. et A. Scott $(1895$, p. 360). Monotypique : Lomanoticola insolens T. et A. Scott 1895. 
30. Macrocheiron auctorum (G.-O. Sars 1918, p. 163). Err. typog. = Macrochiron Brady 1872 .

31. Macrochiron Brady (1872, p. 9). Monotypique : Macrochiron fucicolum Brady 1872.

32. Modiolicola Aurivillius (1882, pp. 39-40). Monotypique : Modiolicola insignis Aurivillius 1882.

33. Monstrilla Dana (1853, p. 1313). Monotypique : Monstrilla viridis Dana 1853.

34. Myicola R.-R. Wright (1885, p. 120). Monotypique : Myicola metisiensis Wright 1885 .

35. Mytilicola A. Steuer (1902, p. 635). Monotypique : Mytilicola intestinalis Steuer 1902.

36. Obesiella W.-G. Ridewood (1903, p. 463). Monotypique : Obesiella lyonsiella Ridewood 1903.

37. Paclabius Kossmann (1877, p. 23). Monotypique : Paclabius tumidus Kossmann 1877.

38. Panaietis Stebbing (1900, p. 666). Monotypique : Panaietis incamerata Stebbing 1900.

39. Paralichomolgus Thompson et A. Scott (1903, p. 281). Génotype par préséance : Paralichomolgus curticaudatus Thompson et A. Scott 1903 .

40. Paranthessius Claus (1889, p. 342). Monotypique : Paranthessius anemoniæ Claus 1889.

41. Pennella Oken (1815, p. 358). Monotypique: Pennatula sagitta Linné 1758 [syn. Pennella diodontis Oken 1815] (1).

42. Pseudanthessius Claus (1889, p. 344). Monotypique : Pseudanthessius gracilis Claus 1889.

43. Pseudolichomolgus Pesta (1909, p. 265). Monotypique : Pseudolichomolgus pectinis Pesta 1909.

44. Pseudomolgus G.-O. Sars (1916, pp. 3, 11). Génotype par désignation : Lichomolgus arenicolus Brady 1880 (= Boeckia arenicola Brady 1872).

45. Sabelliphilus M. Sars (1862, p. 139). Monotypique : Sabelliphilus elongatus M. Sars 1862.

46. Scottocheres Giesbrecht (1897, p. 18). Génotype par désignation: Acontiophorus elongatus Th. et A. Scott 1894.

(1) Oken (1815 p. 358) cite comme seule espèce de son genre Pennella, le « Lerna diodontis Oken "d'Orthagoriscus mola L., qui est l'espếce décrile sous le nom de Pennatula par La Martinière en 1787 et qui avait été récoltée sur Orthagoriscus mola L. de la région de Notka (Californie, côte nord-est). 
47. Sepicola Claus $(1860$, p. 5). Monotypique: Sepicola longicauda Claus 1880.

48. Splanchnotrophus Hancock et Norman (1863, p. 50). Génotype par préséance : Splanchnotrophus gracilis Hancock et Norman 1863. - Bergh $(1876$, p. 408 , note 3 ) a fait remarquer que Splanchnotrophus signifiait exactement le contraire de ce que voulaient exprimer les créateurs du genre, "qui nourrit les viscères » au lieu de «qui se nourrit des viscères »; Bergh ajoute, à titre d'exemple, que Splanchnobroster ou Splanchnobosca, eussent été étymologiquement corrects.

49. Stellicola Kossmann (1877, p. 11). Génotype par préséance : Stellicola thorelli Kossmann 1877.

50. Strongylopleura Pelseneer (1928, p. 39). Monotypique : Strongylopleura histrio Pelseneer 1928.

51. Tanypleurus Steenstrup et Lütken (1861, p. 425). Monotypique : Tanypleurus alcicornis Steenstrup et Lütken 1861. - Considéré à tort par Bassett-Smith (1899, p. 495) comme identique à Ismaïla Bergh 1866.

52. Tococheres Pelseneer (1928, pp. 36-37). Monotypique: Tococheres cylindraceus Pelseneer 1928.

53. Trochicola Dollfus (1914, p. 1528). Monotypique : Trochicola enterica Dollfus 1914. [lapsus pro entericus].

\section{NOMENCLATOR SPECIERUM}

Avant d'aborder l'énumération systématique des espèces. de Copépodes parasites ou commensales de mollusques, il ne sera peutêtre pas inutile de résumer la classification adoptée.

I. CYCLOPIFORMES Oakley 1930

1. Monstrilloida G. O. Sars 1901. Monstrillidae.

2. Calanoida G. O. Sars 1901.

3. Harpacticoida G, O. Sars 190I:

4. Cyclopoida G. O. Sars 1901:

Oilhonidze.

Cyclopinida.

Cyclopida. Ascomyzontidae. Acontiophoridx. Nyzopontiidze. 
Dyspontiidæ.

Artotrogidae.

Cancerillida.

Clausidiidx.

Lichomolgidae.

Oncridx.

Corycaida.

Ergasilidz.

Clausiidae.

Chondracanlhidie.

Splanchnotrophidae.

Eunicicolidie.

5. Notodelphyoida G. O. Sars 1901.

II. CALIGIFORMES Oakley 1930.

Caligidae.

Diche'esthiida.

Sphyriida.

Lernaeidae.

Lernæopodidx.

Chonioslomatidie.

Herpyllobiidx.

Cyclopiformes Oakley (1930, p. 185).

MONSTRILLOIDA G.-O. Sars (1901, p. 2)

MONSTRILLIDA

Monstrilla Dana (1853, p. 1313).

Monstrilla helgolandica Claus 1863

1863. Monstrilla helgolandica Claus, p. 165 , pl. 12 , fig. 15 et 13 , fig. 9 .

1914. Monstrilla helgolandica Pelseneer, pp. 8-14, pl. 3 fig. 1-7. Loc. - Wimereux (Pas-de-Calais).

Hôte. - Odostomia rissoides Hanley. .

Remarques. - Le Monstrilla n'est parasite qu'à l'état larvaire. Pelseneer (1914, pp. 12-13), signale la ressemblance existant entre les « appendices fixateurs et absorbants » de la larve de Monstrilla et ceux de Splanchnotrophus. Il est indubitable que les appendices latéraux des Splanchnotrophus typiques (sous-genre Splanchnotrophus) et ceux des larves de Monstrilla sont morphologiquement tout à fait comparables, ce qui n'implique nullement un rapprochement systématique entre ces genres qui appartiennent à des groupes très différents. 


\section{CYCLOPOIDA G.-O. Sars (1901, p. 2) \\ Ascomyzontida G.-O. Sars (1918, pp. 83-84). \\ Scottocheres Giesbrecht (1899, p. 104). \\ scottocheres elongatus (T. et A. Scott 1894)}

1894. Acontiophorus elongatus T. et A. Scott, pp. 145-146, pl. 9, fig. 15-20.

1899. Scottocheres elongatus Giesbrecht, pp. 17, 82, 104, pl. 4, fig. 1-13.

1903. Scottocheres elongatus Thompson et A. Scott, p. 290.

1906. Scottocheres elongatus Norman et A. Scott, p. 193.

1918. Scottocheres elongatus G.-O. Sars, pp. 107-109, pl. 66.

Loc. - Norvège, Ecosse, Manche (Plymouth), Naples, Ceylan.

Hôte. - Chlamys (Equipecten) opercularis (Linné), (Norman et A. Scott 1906).

Artotrogide Brady ; sensu G.-O. Sars (1918, p. 132)

Artotrogus Boeck (1859, p. 2).

Artotrogus orbicularis Boeck 1859

1859. Artotrogus orbicularis Boeck, pp. 171-175, pl. I, fig. 1-10. 1899. Artotrogus orbicularis Giesbrecht, p. 111, pl. IX, fig. 24-34 et X, fig. 33-35.

1918. Artotrogus orbicularis G. O. Sars, pp. 134-135, pl. 78.

Loc. - Mer de Kara, Norvège, Iles-Britanniques, Ceylan.

Hótes. - Doris sp., Nudibranches indéterminés.

\section{Artotrogus australis Wilson 1923}

1923. Artotrogus australis Wilson, pp. 2-4, pl. I, fig. 1-5.

Loc. - Géorgie du Sud.

Hote. - Probablement Archidoris nivalis Thiele var. antarctica Vayssière.

Obesiella Ridewood (1903, p. 463).

Obesiella lyonsiellæ Ridewood 1903

1903. Obesiella lyonsiellæ Ridewood, pp. 463-465, 2 fig. n. num. Loc. - Partie méridionale de l'Océan Indien (cf. p. 196).

Hòte. - Lyonsiella [? papyracea Edgar A. Smith]. 
Remarques. - Le descripteur place Obesiella dans les Ascomyzontidæ, attribution qui n'est pas invraisemblable mais qui devra être établie par une éțde détaillée des pièces buccales, à peine décrites et pratiquement non figurées. Il faut attirer l'attention sur la ressemblance d'Obesiella et d'Ischnurella Pelseneer 1928, genre qui pourrait même peut-être se révéler synonyme du premier lorsqu'il sera moins imparfaitement connu : on ne sait pour l'instant absolument rien sur son antenne, ses pièces buccales et ses 4 paires antérieures de pattes thoraciques.

\section{LichOMOLGID A}

\section{Lichomolgus Thorell (1860, pp. 64-69).}

Doridicola Leydig 1853. - Sepicola Claus 1860. — Eolidicola M. Sars 1862. - Macrochiron Brady 1872. - Stellicola Kossmann 1877. - Linckiomolgus Stebbing 1900. - Paralichomolgus Thompson et A. Scott 1903.

Remarques. - G. O. Sars (1918, p. 163) a rendu à Macrochiron Brady 1872, le rang générique qu'il avait perdu depuis 1880 (Brady). En fait, les différences qui séparent Lichomolgus de Macrochiron sont extrêmement faibles, la « principale » étant la taille et la forme de la $5^{\circ}$ paire de pattes thoraciques, plus développées que chez Lichomolgus et plus ou moins recourbées en arrière. Il semble donc très suffisant de ne conserver à Macrochiron qu'une valeur sous-générique. Quant au genre Stellicola Kossmann 1877, (dont Linckiomolgus Stebbing 1900 et Paralichomolgus Thompson et A. Scott 1903, sont inséparables), il a été créé pour des Lichomolgus à très forte dilatation céphalo-thoracique et à bords latéraux des somites 2-4 non arrondis et Paralichomolgus a un petit article supplémentaire aux antennules qui sont 8 -articulées. Etant donné qu'il existe des Macrochiron très dilatés, mais à antennule typique, 7-articulée et au moins un Stellicola (orbicularis Monod) à somites thoraciques 2-4 latéralement arrondis, on ne peut pas considérer Stellicola comme un genre distinct mais tout au plus comme un sous-genre de Lichomolgus.

Le déplacement de Lichomolgus au profit de Doridicola, légitime en théorie, serait un admirable exemple d'application mécanique, aveugle, irraisonnée et malfaisante de la loi de priorité. D'ailleurs malgré son indiscutable priorité la description de Leydig est si médiocre que, s'il ne s'était agi d'une espèce parasite qui a pu être retrouvée grâce à l'indication d'hôte, Doridicola agilis serait demeuré une species inquirenda. Quant à Sepicola, on ignore s'il a la priorité 
sur Lichomolgus, et, l'aurait-il, qu'il serait néfaste de le rétablir étant donné l'unanimité de l'adoption de Lichomolgus.

\section{Sous-genre Lichomolgus}

Doridicola Leydig 1853. - Sepicola Claus 1860. - Eolidicola M. Sars 1862 .

Remarques. - Le sous-genre Lichomolgus comprend les Lichomolgus typiques, à antennules 7 -articulées, à endopodite de la $4^{\circ}$ paire de pattes toujours 2 -articulé, à $5^{\circ}$ paire de pattes relativement courte ou très courte, à céphalothorax jamais très dilaté. - Le sousgenre contient de nombreuses espèces dont quelques-unes ont été trouvées sur des Mollusques.

Lichomolgus (Lichomolgus) agilis (Leydig 1853) Canu 1892

Fig. $3 \mathrm{~B} ; 8$.

1853. Doridicola agilis Leydig, pp. 377-382, pl. XIV, fig. 1-8.

1862. Eolidicola tenax M. Sars, p. 138.

1875. Doridicola sp., Claus, p. 348, pl. XXIX, fig. 29.

1880. Lichomolgus chromodoridis Della Valle, pp. 120-121, pl. II, fig. $43-48$.

1881. Lichomolgus chromodoridis Della Valle, pp. 101-102, pl. VI, fig. 43-48.

1882 a. Doridicola agilis : Aurivillius, pp. 34-38, pl. V, fig. 1-12.

1882 b. Lichomolgus agilis : Aurivillius, pp. 42-43, pl. XIII, fig. 9.

1889. Lichomolgus doridicola Claus, p. 12, pl. II, fig. 1-12.

1891. Lichomolgus doridicola: Canu, p. 478.

1892. Lichomolgus concinnus Th. Scott, p. 261, pl. XI, fig. 25-33.

1892. Lichomolgus agilis : Canu, pp. 228-230, pl. XXII, fig. 1-14.

1893. Lichomolgus concinnus Th. et A. Scott, p. 212, pl. VII, fig. 12-15.

1894. Lichomolgus agilis: Canu, p. 3.

1896. Lichomolgus doridicola: Hecht, pp. 86-87.

1899. Lichomolgus doridicola: Canu, p. 73.

1918. Lichomolgus agilis : G.-O. Sars, p. 162, pl. XC.

Loc. - Mer du Nord, Manche, Golfe de Gascogne, Méditerranée. - Còte Atlantique du Maroc : "Vanneau » Station LXVII (Lat. N., $30^{\circ} 34^{\prime} 30^{\prime \prime}$, Long. W. G. : 949'30”), 21. VIII. 1925 , prof. 55 m., vase ; entre les plis branchiaux de Armina verrucosa (Cantraine), R.-Ph. Dollfus coll. (triage des matériaux récoltés par J. Liouville et R.Ph. Dollfus). 
Hôtes. - Eolidia papillosa (Linné), Facelina longicornis (Montagu) Facelina curta (Alder et Hancock), Janolus hyalinus (Alder et Hancock), Janolus cristatus (Delle Chiaje), Protonotus mucronifer (Alder et Hancock), Idula coronata (Gmelin), Armina verrucosa (Cantraine), Sphærostoma hombergi (Cuvier), Staurododris verrucosa (Cuvier) Bergh, Archidoris tuberculata (Cuvier), Archidoris britannica (Johnston) Bergh, Thordisa millegrana (Alder et Hancock) Bergh, Jorunna tomentosa (Cuvier), Glossodoris elegans (Cantraine), Dendrodoris lugubris (Gravenhorst), Euphurus claviger (O. F. Müller), Palio lessoni (d'Orbigny) Bergh, Polycera sp., Doridopsis limbata (Cuvier).

\section{Lichomolgus (Lichomolgus) longicauda (Claus 1860)}

1860. Sepicola longicauda Claus, pp. 4-5, pl. I, fig. 8-9.

1875. Lichomolgus sepicola Claus, p. 348.

1877. Lichomolgus sepicola Wierzejski, p. 574, pl. 34.

1909. Lichomolgus sepicola Pesta, p. 262-263, pl. II, fig. 7.

1927. Lichomolgus longicauda Cuénot, p. 287.

Loc. - Adriatique (Trieste), Golfe de Gascogne (Arcachon).

Hôtes. - Sepia officinalis Linné, Sepia filliouxi Lafont.

Remarques. - Claus n'avait aucune raison, parce qu'il plaçait son Sepicola longicauda dans le genre Lichomolgus de créer un nonveau nom spécifique.

Lichomolgus (Lichomolgus) sp.

Fig. $2 \mathrm{C} ; 3 \mathrm{~A} ; 4 \mathrm{~A}$; 5 D ; 6 B-C, G-H

1929. [Copépode commensal] Risbec, p. 296, fig. 60.

Loc. - 2 \%, sur Aeolidia nebæ Risbec, Ile Mouac, Nouvelle-Calédonie, J. Risbec coll.

Remarques. - Nous nous contentons de donner quelques figures de cette espèce trop mal connue pour qu'il soit possible de la rattacher en toute certitude à une forme décrite ou pour en faire le type d'une espèce nouvelle.

Sous-genre Macrochiron Brady 1872.

Lichomolgus auctorum pro parte

Remarques. - Le sous-genre Macrochiron comprend les Lichomolgus à antennules 7-articulées, à endopodite de la $4^{\circ}$ paire de pat- 
tes thoraciques 1 - ou 2 -articulé, à $5^{\circ}$ paire de pattes relativement longue et le plus souvent recourbée en arrière, à céphalothorax parfois très dilaté. - G. O. Sars ayant montré (1918) que les Lichomolgidés à endopodite de la $4^{\circ}$ patte thoracique 1 -articulé ne sont pas forcément des Pseudanthessius mais peuvent dans certains cas être rapportés à Macrochiron, il en résulte que « several of the exotic species described by $\mathrm{A}$. Scott and referred by him some to the genus Lichomolgus and some to the genus Pseudanthessius, may more properly be included in the present genus » (1918, p. 163). A notre avis, et pour autant que nous en pouvons juger d'après les seuls documents publiés, on peut énumérer de la sorte les espèces décrites par Thompson et A. Scott en 1903 (Ceylan), et par A. Scott en 1909 (iles de la Sonde).

1903. - Pseudanthessius maximus = Lichomolgus (Macrochiron) maximus; Pseudanthessius gracilis Claus, ne change pas; Pseudanthessius chelifer $=$ Lichomolgus (Macrochiron) chelifer ; Pseudanthessius concinnus ne change pas; Lichomolgus gracilis, L. ieversi, L. lankensis, L. simplex, L. robustus et L. gigas ne changent pas ; Lichomolgus buddhensis = Lichomolgus (Macrochiron) buddhensis ; Lichomolgus elegans $=$ Lichomolgus (Macrochiron) elegans ; Lichomolgus dentipes $=$ Lichomolgus (Stellicola) dentipes ; Paralichomolgus curticaudatus $=$ Lichomolgus (Stellicola) curticaudatus ; Paralichomolgus longicaudatus $=$ Lichomolgus (Stellicola) semperi Kossmann 1877.

1909. - Lichomolgus anomalus $=$ ? Lichomolgus (Macrochiron) albus ; Lichomolgus gracilipes $=$ Lichomolgus (Macrochiron) gracilipes; Pseudanthessius weberi, $P$. pectinatus, $P$. obscurus ne changent pas ; Pseudanthessius parvus $=$ Lichomolgus (Macrochiron) parvus; Herrmannella concinna $=$ Anthessius concinnus.

\section{Lichomolgus (Macrochiron) gracilipes A. Scott 1909}

1909. Lichomolgus gracilipes A. Scott, pp. 265-266, pl. LXVIII, fig. 11-20.

Loc. - Rotti Island, iles de la Sonde.

Hôte. - L'espèce a été recueillie sur un nudibranche non spécifié, mais qui est un des suivants : Hexabranchus marginatus (Quoy et Gaimard), Glossodoris annæ (Bergh), Gymnodoris rubromaculata (Bergh).

Remarques. - Espèce extrêmement voisine des deux suivantes (alabatensis et Macrochiron sp.). 
Lichomolgus (Macrochiron) alabatensis (Kossmann 1877) Fig. $2 \mathrm{D} ; 4 \mathrm{~B} ; 6 \mathrm{D} ; 9 \mathrm{~A}-\mathrm{B}$.

1877. Stellicola alabatensis Kossmann, pp. 14-15, pl. I, fig. 2-3. 1929. [Copépode commensal] Risbec, p. 290.

Loc. - 1 \&, 1 ơ, sur Gymnodoris kouaouæ (Risbec), Nouméa, Nouvelle-Calédonie, J. Risbec coll. « Le Copépode semble gêner considérablement le Trevelyana [Gymnodoris]. Lorsqu'il arrive dans les branchies, celles-ci se rétractent brusquement. Il en est de même pour les rhinophores ». (J. Risbec, in litt., 3.XII.1928).

Hab. - Philippines (Semper coll.).

Remarques. - Le Stellicola alabatensis est fort mal connu, décrit en dix lignes par Kossmann d'après deux croquis ( $\sigma^{\star}$ et o $^{\text {) }}$ de Semper. La forme de Stellicola alabatensis étant tout à fait semblable à celle du parasite de Trevelyana, on peut considérer l'identification comme vraisemblable, et en tous les cas comme inoffensive, étant donné qu'alabatensis est à peine davantage qu'un nomen nudum et que le type n'en existe pas. Le parasite de Gymnodoris n'est pas sans rapports avec Lichomolgus gracilipes A. Scott 1909 (pp. 265266, pl. LXVIII, fig. 11-20) qui a été recueilli sur un Nudibranche des îles de la Sonde, mais cette espèce est plus étroite et a sa dilatation maxima très antérieure en comparaison d'alabatensis.

\section{Lichomolgus (Macrochiron) sp.}

Fig. $2 \mathrm{E} ; 5 \mathrm{C}, \mathrm{E} ; 6 \mathrm{~A} ; 7$.

1929. [Copépode commensal] Risbec, p. 281, fig. 22.

Loc. - 1 q, sur Chromodoris mouaci Risbec, Ile Mouac, NouvelleCalédonie, J. Risbec coll. «Copépodes, avec sacs à œufs, violets comme l'hôte. Le Nudibranche s'est mis à pondre le 8 octobre [1928]; un Copépode femelle est resté posé longtemps près de l'orifice de ponte. Sur un animal en marche, un Copépode posé sur les côtés du pied avait des œufs d'aspect anormal, échevelés. Il en partait comme des éclairs qui seraient des larves qui montaient vers la surface ». (J. Risbec, in litt., 3.XII.1928).

Remarques. - Ce spécimen est extrêmement voisin du parasite de Gymnodoris. Il existe cependant une différence qui nous oblige à en distinguer le parasite de Chromodoris : chez celui-ci les deux griffes terminales de l'antenne sont très inégales, tandis qu'elles sont sensiblement égales chez les spécimens provenant de Gymnodoris. 


\section{Lichomolgus (Macrochiron) trochi (Canu 1899)}

Fig. $1 \mathrm{~A}-\mathrm{F}$; 24 G.

1899. Lichomolgus trochi Canu, pp. 75-79, pl. VIII.

1927. Lichomolgus trochi Dollfus, pp. 120-121.

1928. Lichomolgus trochi Pelseneer, p. 44.

D'après un spécimen récolté par R.-Ph. Dollfus dans la cavité

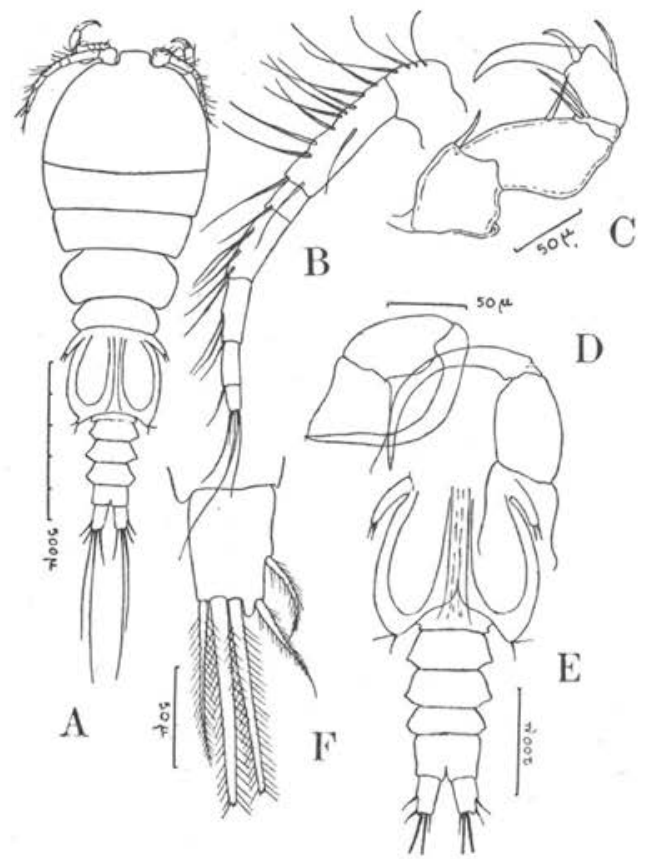

Fıg. 1. - Lichomolgus trochi Canu, mâle. A, Vue dorsale du spécimen ; B, Antennule; C, Antenne : D, maxillipèdes; E, Abdomen ; F, branche furcale.

-palléale de Gibbula cineraria Linné à Roscoff, Finistère (25 sept.3 oct. 1913). Nous figurons le mâle qui n’a pas encore été signalé.

Que l'on accepte Macrochiron Brady 1872, comme genre ou sousgenre, il est évident que Lichomolgus trochi doit en faire partie : la $5^{\circ}$ patte thoracique (fig. $1 \mathrm{E}$ et $24 \mathrm{G}$ ) est, en effet, longue et un peu incurvée.

Sous-genre Stellicola Kossmann 1877.

Linckiomolgus Stebbing 1900. - Paralichomolgus Thompson et A. Scott 1903 . 
Remarques. - Le sous-genre Stellicola comprend les Lichomolgus à antennules 8-articulées (toujours ?), à endopodite de la $4^{\circ}$ paire de pattes 2 -articulé, à $5^{\text {e }}$ paire de pattes relativement développée, pouvant être recourbée en arrière, à céphalothorax toujours très dilaté et à bords latéraux des somités thoraciques 2-4 aigus, bi-dentés, denticulés, très rarement arrondis (orbicularis).

Linckiomolgus Stebbing 1900 est synonyme de Stellicola Kossmann 1877. Il suffit, pour s'en convaincre, de comparer les figures

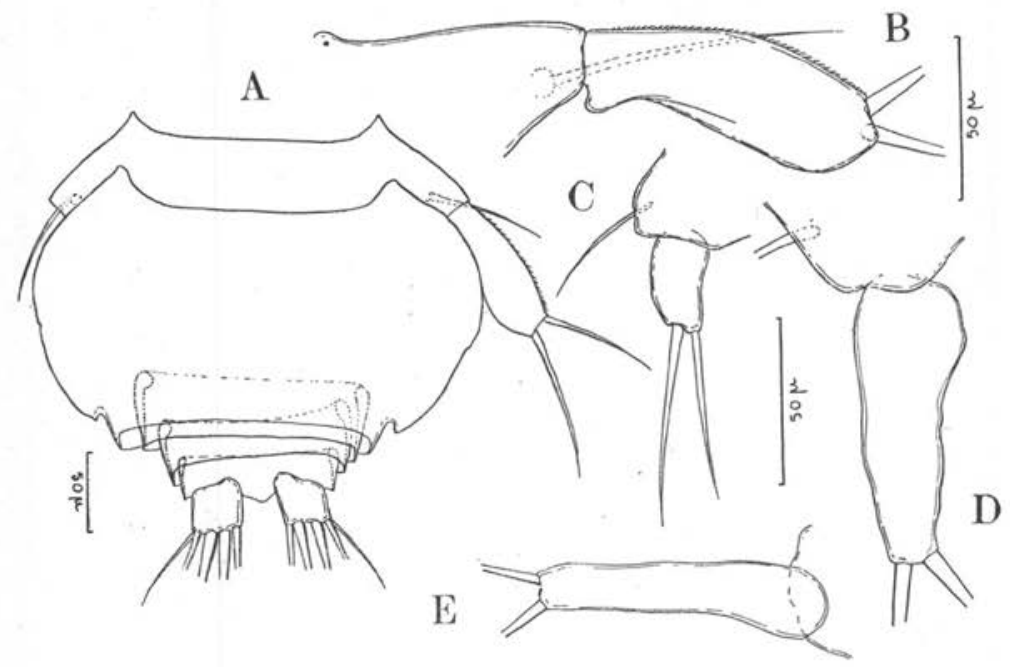

Fig. 2. - A, Lichomolgus (Slellicola) orbicularis (Monod), ९, abdomen ; B, ld., $5^{*}$ patte thoracique; C, Lichomolgus (Lichomolgus) sp., ${ }^{+}$, parasite d'Acolidia, nebae Risbec, $5^{\mathrm{e}}$ patte thoracique; D, Lichomolgus (Macrochiron) alabatensis) (Kossmann), \&, $5^{\mathrm{e}}$ patte thoracique; E, Lichomolgus (Macrochiron) sp., parasite de Chromodoris monaci Risbec, $5^{\mathrm{e}}$ patte thoracique.

données par les auteurs de L. crruleus et de $S$. semperi (Kossmann, 1877, fig. texte p. 14 et pl. III, fig. 1 ; Stebbing, 1900, pl. LXXIV, fig. B). Les figures de l'espèce in copula (Kossmann, 1877, fig. texte p. 14 et Stebbing, 1900, pl. LXXIV, fig. B ơ q) sont particulièrement convaincantes. Elles établissent même, à mon avis, non seulement l'identité générique de Linckiomolgus et de Stellicola mais la conspécificité de $L$. cæruleus et de $S$. semperi, provenant respectivement de Linckia sp., Feather Island, China Straits, Nouvelle-Guinée et d'Ophidiaster miliaris (1), Isabella, Philippines. Paralichomolgus Thompson et A. Scott 1903, est aussi un synonyme puisque P. lon-

(1) Actuellement Linckia miliaris (Linck). 
gicaudatus paraît même être identique à Stellicola semperi (= Linckiomolgus cæruleus) (Fig. 10 A-E).

Lichomolgus (Stellicola) pleurobranchi Kossmann 1877

1877. Stellicola pleurobranchi Kossmann, pp. 15-16, pl. III, fig. 3 . Loc. - Archipel Palaos.

Hôte. - Pleurobranchus sp.

Lichomolgus (Stellicola) orbicularis (Monod 1928)

Fig. 2 A-B ; 5 A-B ; 6 E-F.

1928. Paralichomolgus orbicularis Monod, pp. 2-8, fig. 24. 1928. Paralichomolgus orbicularis Risbec, p. 35, fig. 1 ter.

Loc. - Nouméa, Nouvelle-Calédonie.

Hôte. - Platydoris cruenta (Quoy et Gaimard).

\section{Paranthessius Claus 1889.}

Herrmannella Canu 1891. - Pseudolichomolgus Pesta 1909.

Remarques. - Canu (1892) qui n'avait à sa disposition qu'une seule espèce d'Herrmannella, distinguait ce genre de Paranthessius Claus 1889, par trois caractères: morphologie des antennes, des maxilles et de la $4^{\circ}$ paire de pattes thoraciques.

$1^{\circ}$ Antennes. Bien que, dans les deux cas, l'article 3 (pénultième) soit court (alors qu'il est long chez Modiolicola), les antennes d'Herrmannella rostrata et de Paranthessius anemoniæ sont assez différentes, trapues et surmontées d'une forte griffe dans le premier cas, plus grêles et terminées par un certain nombre de fortes soies spiniformes arquées dans le second. On ne saurait voir là de caractère générique puisqu'il existe entre les antennes de Lichomolgus (Lichomolgus) agilis Leydig et Lichomolgus (Lichomolgus) tenuifurcatus G.-O. Sars, par exemple, les mêmes différences que l'on observe entre les antennes d'Herrmannella rostrata et de Paranthessius anemonix. D'ailleurs parmi les espèces norvégiennes que G.-O. Sars considère comme congénériques et place dans le genre Herrmannella, on trouve des antennes très variées, tantòt très robustes, uncifères $(H$. valida G.-O. Sars, $H$. prehensilis G.-O. Sars) comparables à. celle d'Herrmannella rostrata, tantôt grêles et terminées par des soies fortes, mais sans griffe $(H$. parva Norman et A Scott, H. finmarchica A. Scott, H. tenuicaudis G.-O. Sars). 
$2^{\circ}$ Maxilles $\left[\mathrm{mx}^{2}\right.$, « maxillipède antérieur », etc. $]$. Comme pour les antennes, il y a ici une différence manifeste entre Herrmannella rostrata et Paranthessius anemonix sans qu'il soit possible de lui accorder une importance d'ordre générique : la maxille d'Hermannella rostrata comprend un axe principal pectiné, une phanère

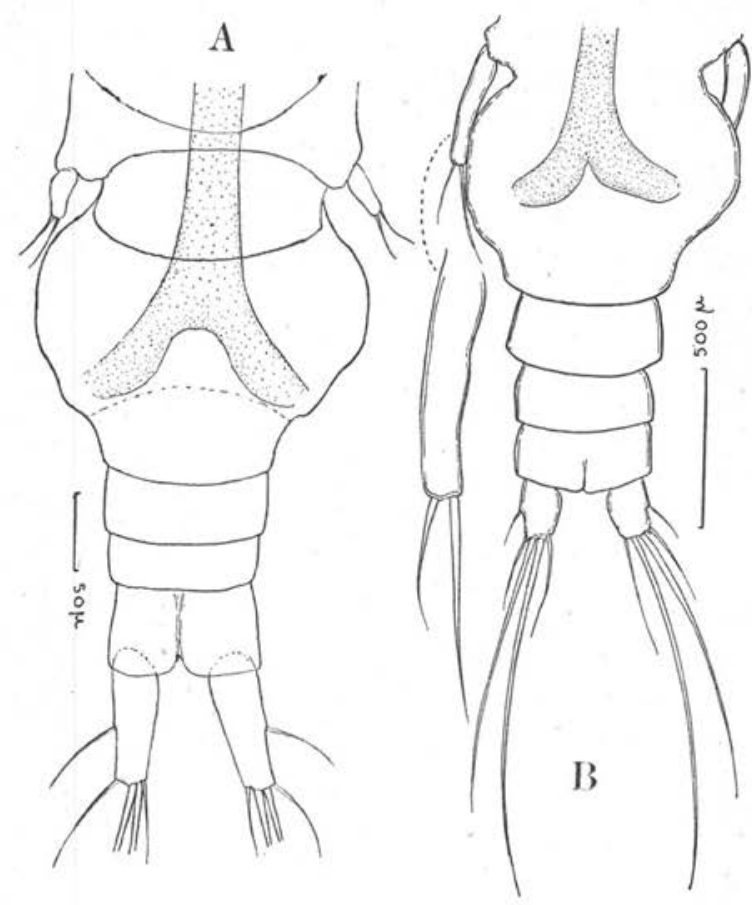

Fig. 3. - A, Lichomolgus (Lichomolgus) sp., ᄋ, parasite d'Aeolidia nebae Risbec, abdomen; B, Lichomolgus (Lichomolgus) agilis (Leydig),, , parasite d'Armina verrucosa (Cantraine), abdomen .

accessoire (Seitenborste Claus, 1889) pectinée et sensiblement aussi longue, enfin une phanère basale (Nebenborste Claus, 1889) glabre et très courte. Chez Paranthessius anemonix, les deux premiers éléments sont moins incurvés, moins effilés, plus courts, et surtout la phanère basale glabre atteint presque les dimensions des éléments pectinés. Remarquons que dans l'intérieur du genre Lichomolgus et du sous-genre Lichomolgus (d'après les planches de G.-O. Sars 1918), on observe des maxilles qui ne sont nullement construites sur un type absolument uniforme : chez L. agilis Leydig, par exemple, on trouve l'axe principal pectiné, la phanère accessoire pectinée 
et la phanère basale glabre et réduite, mais chez $L$. tenuifurcatus G.-O. Sars, L. canui G.-O. Sars, L. furcillatus Thorell (1), L. marginatus Thorell, L. forficula Thorell, on ne trouve, à côté de l'axe principal pectiné, qu'une petite phanère pectinée (peut-être une "phanère basale» ici non glabre). Si l'on examine les espèces placées par G.-O. Sars dans le genre Herrmannella on constatera que toutes - sauf peut-être tenuicaudis - n'ont, comme les
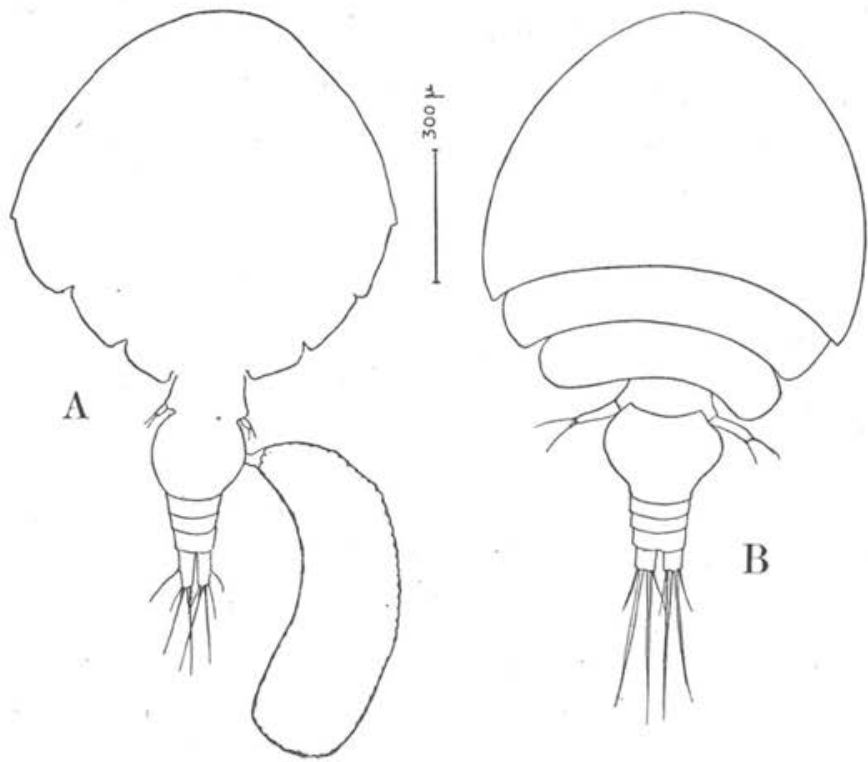

FIG. 4. - A, Lichomolgus (Lichomolgus) sp.. \&, parasite d'Aeolidia nebae Risbec, spécimen probablement assez déformé ; B, Lichomolgus (Macrochiron) alabatensis (Kossmann), $q$.

Lichomolgus que nous venons de citer, qu'une petite phanère (pectinée) à la base de l'axe principal.

$3^{\circ}$ Quatrième paire de pattes thoraciques. Sans doute les trois articles de l'endopodite portent, chez Herrmannella rostrata respectivement $1+1+2$ soies ou épines, tandis que le nombre est, chez Paranthessius anemonia (fide Claus, 1889, pl. IV, fig. 15), de $1+2+3$. Pas plus que pour les antennes ou les maxilles il n'y a là de caractère générique : alors que certains Herrmannella (rostrata Canu, valida G.-O. Sars, parva Norman et A. Scott) ont

(1) Placé par Norman et A. Scott (1906, p. 198) dans le genre Psendanlhessius, certainement à tort. 
$1+1+2$, d'autres (prehensilis G.-O. Sars, tenuicaudis G.-O. Sars et finmarchica A. Scott) ont $1+1+5$.

Ajoutons que le rostre recourbé et postérieurement pointu d'Herrmannella "rostrata " existe chez Paranthessius : "Rostrum gestreckt, schmal und nach hinten verjüngt »(Claus, 1889, p. 16).

Il semble résulter de ces remarques que la validité d'Herrmannella

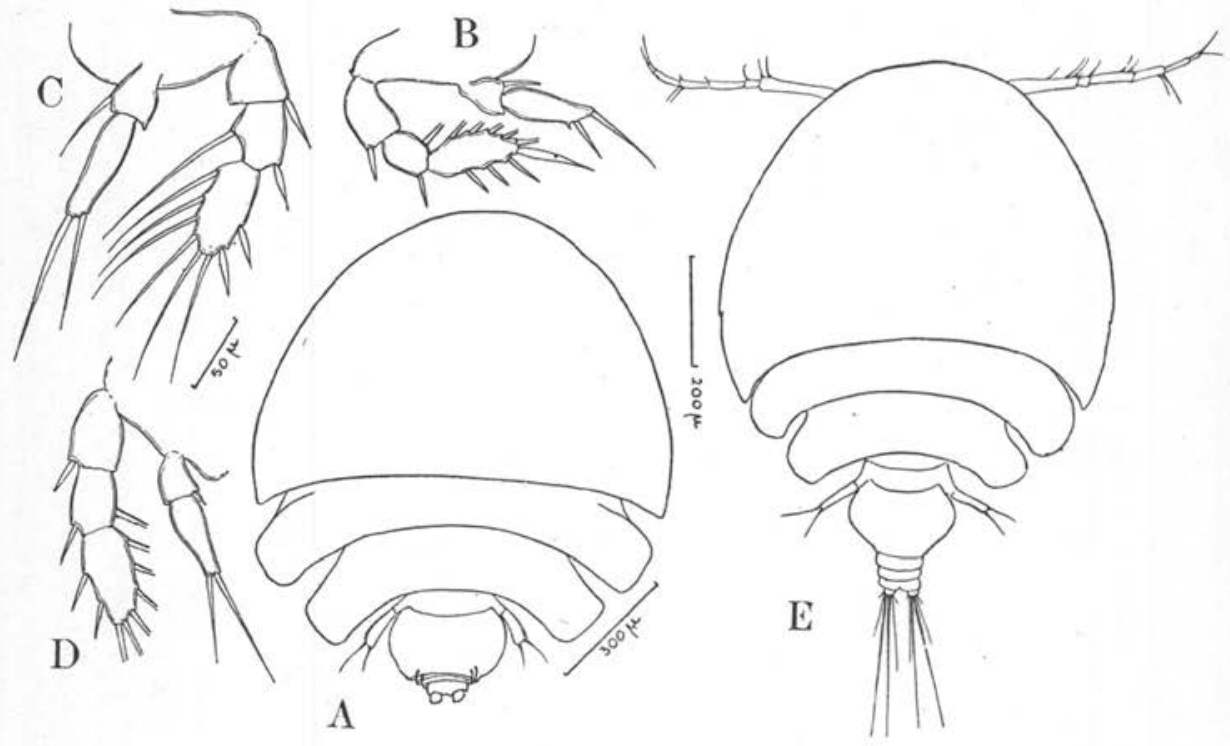

FIG. 5. - A, Lichomolgus (Slellicola) orbicularis (Monod), Q; B. Id. 4" patte thoracique ; C, Lichomolgus (Macrochiron) sp.,, , parasite de Chromodor's mouaci Risbec, $4^{e}$ patte thoracique: D, Lichomolgus (Lichomolgus) $\mathrm{sp..}$, , parasite d'Aeolidia nebae Risbec, $4^{\text {e }}$ patte thoracique ; E, Lichomolgus (Macrochiron) sp., , parasite de Chromodoris mouaci Risbec.

Canu ne parait pas assise sur des caractères assez importants pour pouvoir être maintenue et qu'il faut considérer ce genre comme synonyme de Paranthessius Claus.

Considérant avec G.-O. Sars qu'il n'est pas possible de séparer génériquement les formes à maxille bifide (Herrmannella sensu G.-O. Sars) des formes à maxille trifide (Herrmannella Canu, Paranthessius Claus), il est évident que Pseudolichomolgus Pesta doit être considéré comme synonyme de Paranthessius Claus $(=$ Herrmannella Canu). 


\section{Paranthessius rostratus (Canu 1891)}

1891. Herrmannella rostrata Canu, p. 480.

1891 a. Herrmannella rostrata Canu, p. 436.

1892. Hermannella rostrata Canu, pp. 236-237, pl. XXIV, fig. 1-13. 1892. Lichomolgus agilis Th. Scott, pp. 266-267 (nomen nudum).

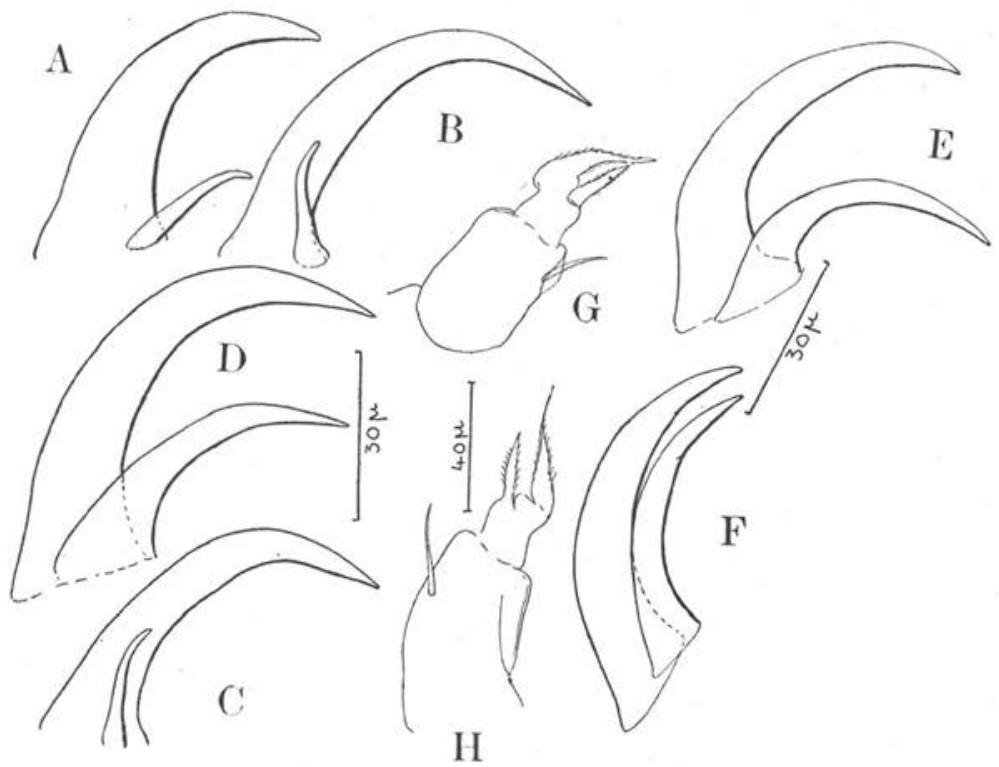

Fig. 6. - A, Lichomolgus (Macrochiron) sp., $Q$, parasite de Chromodoris mouaci Risbec, extrémité antennaire; B, C, Lichomolgus (Lichomolgus) sp., Q parasite) d'Aeolidia nebae Risbec, extrémitê antennaire; D. Lichomolgus (Macrochiron alabalensis Kossmann, Ǫ extrémité antennaire; E, F, Lichonolgus (Stellicola) orbicularis (Monod), Q, extrémité antennaire; G, H, Lichomolgus (Lichomolgus) sp., $\odot$, parasite d'Aeolidia nebae Risbec, maxillipède.

1892. Lichomolgus agilis Th. et A. Scott, pp. 201-203, pl. XV, fig. 1-14.

1893. Lichomolgus agilis Thompson, pp. 207-208, pl. XXV, fig. $8 d$. 1894. Hermannella rostrata Canu, p. 3.

1899. Herrmannella rostrata Canu, p. 73.

1928. Herrmannella rostrata Pelseneer, p. 43.

Loc. - Manche (Boulonnais), Lancashire, Firth of Forth.

Hôtes. - Chlamys (Aquipecten) opercularis (Linné), Mactra corallina (Linné), Cardium edule Linné, Tapes pullaster (Montagu). Remarques. - Canu a signalé dès 1894 (p. 10, note 4) que Licho- 
molgus agilis Th. et A. Scott (nec Leydig!) = Herrmannella rostrata Canu.

\section{Paranthessius pectinis (Pesta 1909)}

1909. Pseudolichomolgus pectinis Pesta, pp. 263-265, pl. II, fig. 8-10 et III, fig. 11-16.

Loc. - Golfe de Barcola, près Trieste, Adriatique.

Hôte. - Pecten glaber (Linné).

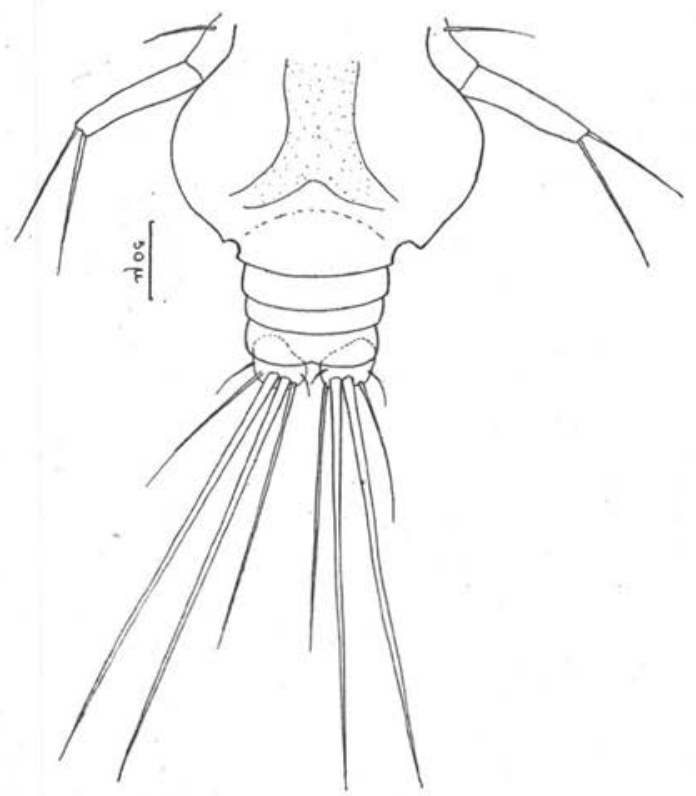

Fig. 7. - Lichomolgus (Macrochiron) sp., $\odot$, parasite de Chromodoris mouaci Risbec, abdomen (considérablement raccourci par la position défectueuse de l'objet dans la préparation).

Anthessius Della Valle 1880.

Boeckia Brady 1872 [præoc. : Boeckia Malm, Efv. Vet. AK. 1870, p. 543 (Amphipode)]. - Pseudomolgus G.-O. Sars 1916.

Remarques. — G.-O. Sars (1918, p. 182) ayant avoué lui-même que le génotype de Pseudomolgus était Lichomolgus arenicola Brady, nous n'avons aucun scrupule à immerger Pseudomolgus dans la synonymie d'Anthessius puisque Lichomolgus arenicola était dès 
1889 (p. 16) reconnu par Claus pour un Anthessius. Il faut signaler aussi que Norman et A. Scott (1906, p. 201) placent Anthessius aux côtés d'Ergasilus et d'Hersiliodes dans les Clausidiidæ, alors qu'il ne semble y avoir aucune raison d'enlever le genre des Lichomolgidx.

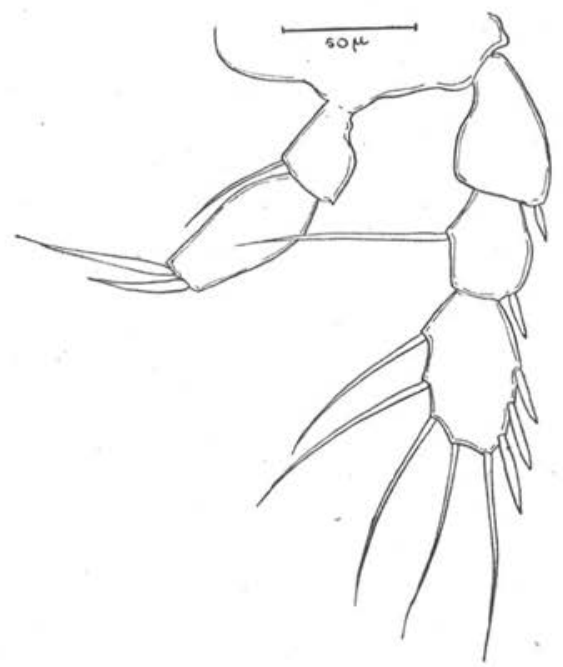

Fıc. 8. - Lichomolgus (Lichomolgus) agilis (Leydig) $\odot$, parasite d'Armina verrucosa (Cantraine), 4" patte thoracique.

Anthessius arenicola (Brady 1872)

1872. Boeckia arenicola Brady, pp. 6-7. 1872 a. Boeckia arenicola Brady, p. 130.

1880. Lichomolgus arenicolus (sic) Brady, p. 46, pl. LXXXVI, fig. 1-7.

1894. Anthessius arenicolus (sic) Canu, pp. 2-9, pl. I, fig. 1-10, et II, fig. 1-7.

1899. Anthessius arenicolus (sic) Canu, p. 73.

1906. Herrmannella arenicola Norman et A. Scott, p. 199.

Loc. - Manche (Boulonnais, Plymouth).

Hôtes. - Buccinum undatum Linné.

Anthessius solenocurti Della Valle 1880

1880. Anthessius solecurti Della Valle, pp. 122-123, pl. II, fig. 49-55. 
1881. Anthessius solecurti Della Valle, pp. 102-104, pl. VI, fig. 49-55.

1894. Anthessius solecurti Canu, p. 9.

1906. Anthessius solecurti Norman et A. Scott, p. 201.

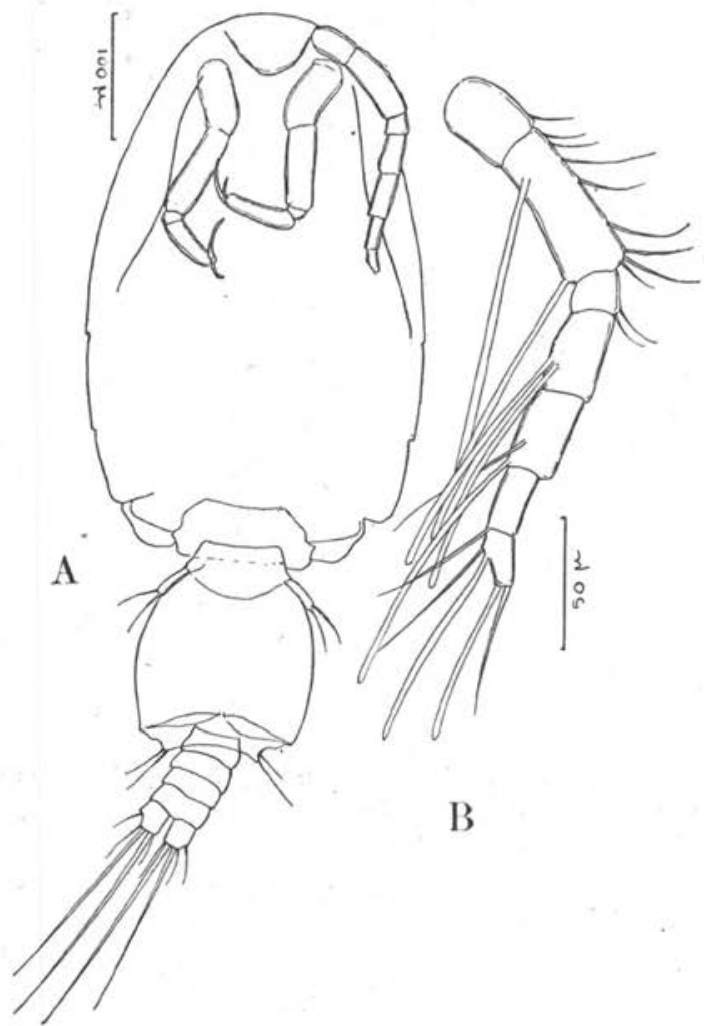

FIG. 9. - A, Lichomolgus (Macrochiron) alabatensis (Kossmann), O๋, en vue dorsale (soies antennulaires, pièces buccales et pattes thoraciques 1-4 non figurées); B, Id., antennule.

Loc. - Manche (Boulonnais, Plymouth), Méditerranée.

Hòtes. - Solenocurtus strigillatus (Linné), Ensis siliqua (Linné). Remarques. - L'orthographe correcte du nom de l'hôte des échantillons typiques étant Solenocurtus on doit considérer la graphie solecurti comme erronée et la remplacer par solenocurti.

\section{Anthessius pleurobrancheæ Della Valle 1880}

1880. Anthessius pleurobranchex Della Valle, p. 123, pl. II, fig. 56-58. 
1881. Anthessius pleurobranchi Della Valle, p. 104, pl. VI, fig. 56-58.

1889. Anthessius pleurobranchi Claus, pp. 15-16, pl. III, fig. 8-13. Loc. - Méditerranée, Naples (?), Trieste.

Hotes. - «Pleurobranchus marmoratus », Pleurobranchea meckeli (Blainville) Leue.

Remarques. - Au sujet de l'identité des espèces de Della Valle et de Claus, cf. Canu 1894 , pp. 4 et passim.

Anthessius spinosus (Raffaele et Monticelli 1885)

1885. Lichomolgus (Sabelliphilus) spinosus Raffaele et Monticelli, pp. 303-305, pl. fig. 1-12.

1894. Anthessius spinosus Canu, pp. 4 et passim.

Loc. - Méditerranée.

Hóte. - Mytilus galloprovincialis Lamarck.

Anthessius hawaïensis (Wilson 1921)

1921. Pseudomolgus hawaïensis Wilson, pp. 13-14, pl. 4, fig. 3538 , pl. 5, fig. 44-48, pl. 6, fig. 49-57.

Loc. - Iles Hawaï.

Hòte. - Pleurobranchus sp.

\section{Anthessius sp.}

1921. Pseudomolgus sp. Wilson, p. 15.

Loc. - Californie.

Hòte. - Tectibranche indéterminé.

Anthessius sp,

1923. Pseudomolgus sp. Wilson, p. 4.

Loc. - Géorgie du Sud.

Hôte. - Archidoris nivalis (Thiele) var. antarctica Vayssière.

Modiolicola Aurivillius 1882.

Remarques. - Modiolicola est extraordinairement voisin de Paranthessius $[=$ Herrmannella $]$. Les différences signalées par Canu dans la morphologie de la maxille ne semblent pas pouvoir être retenues depuis qu'on connaît des Herrmannella dont la maxille ne porte qu'une seule soie accessoire et un Modiolicola dont 
le même appendice en a deux (Wilson, 1921, p. 7, fig. 61). En fait, d'après G. O. Sars (1918, p. 175), les quatre caractères permettant d'opposer Modiolicola à Paranthessius seraient :

$1^{\circ}$ des téguments moins fortement chitinisés, caractère sans valeur générique ;

$2^{\circ}$ l'absence de rostre net, recourbé et postérieurement pointu ;

$3^{\circ}$ le développement de l'article III des antennes (pénultième) toujours court chez Paranthessius ;

$4^{\circ}$ le maxillipède réduit, inerme (ou presque), à article apical tuberculiforme, plus ou moins arrondi.

\section{Modiolicola insignis Aurivillius 1882}

1882 a. Modiolicola insignis Aurivillius, pp. 40-44, pl. VI, fig. 1-10.

1882 b. Modiolicola insignis Aurivillius, pp. 43-48, pl. XIII, fig. 1-8.

1885. Lichomolgus insignis Raffaele et Monticelli, pp. 302, 306, pl., fig. 13-16.

1891. Modiolicola insignis Canu, p. 482.

1892. Modiolicola insignis Canu, pp. 238-240, pl. XXIV, fig. 14-28. 1899. Modiolicola insignis Canu, p. 73.

1918. Modiolicola insignis G. O. Sars, pp. 173-174, pl. XCVII.

Loc. - Norvège, Côtes d'Angleterre, Manche, Méditerranée.

Hötes. - Modiolus modiolus (Linné) Lamarck, Mytilus edulis Linné, Mytilus galloprovincialis Lamarck.

\section{Modiolicola inermis Canu $1891 a$}

1891 a. " Lichomolgide nouveau » [Modiolicola sp.] Canu, p. 436. 1892. Modiolicola inermis Canu, pp. 240-241, fig. 19.

1893. Lichomolgus maximus Thompson, pp. 208-210, pl. XXXV, fig. 1-11.

1894. Modiolicola inermis Canu, pp. 10-14, pl. III, fig. 1-7.

1897. "(?) Lichomolgus maximus » Th. Scott, p. 154.

1899. Modiolicola inermis Canu, p. 74.

1906. Hermannella maxima Norman et A. Scott, p. 199.

Loc. - Manche (Boulonnais, St-Marcouf, Plymouth), Ecosse, Ile de Man.

Hôtes. - Chlamys (Equipecten) opercularis (Linné), Pecten maximus (Linné).

Remarques. - Canu signalait dès 1894 (p. 10) l'identité de Lichomolgus maximus Thompson et de Modiolicola inermis; il se féli- 
cite de cette identité «parce qu'elle fait disparaitre dans la synonymie l'appellation spécifique si peu justifiée du L. maximus Thompson » qui n'est nullement un géant parmi les Lichomolgus (sensu lato). On peut se demander si Thompson n'aurait pas tout simplement voulu rappeler le nom spécifique de l'hôte, en négligeant d'ailleurs de le mettre au génitif (maximi, sous-entendu : Pectinis). - On doit signaler ici que Modiolicola n'est pas toujours parasite de Mollusques, puisque $M$. jamaicensis Wilson 1924 a été obtenu « from black ascidians common upon the mangrove roots on the Bogue Islands, Montego Bay, Jamaica» (p. 15).

\section{Pseudanthessius Claus 1889.}

\section{Pseudanthessius thorelli (Brady 1880)}

1876. Lichomolgus thorellii Brady et Robertson, p. 197 (nomen nudum.

1880. Lichomolgus thorellii Brady, pp. 47-49, pl. LXXXVIII, fig. 1-9.

1906. Pseudanthessius thorelli Norman et A. Scott, p. 198.

Loc. - Plymouth et Salcombe.

Hôte. - Chlamys (Equipecten) opercularis (Linné).

\section{Cholidya Farran 1914.}

Gholidya polypi Farran 1914

1914. Cholidya polypi Farran, pp. 473-475, pl.

Loc. - Au large du S.-W. de l'Irlande, 600-700 fathoms.

Hôte. - Benthoctopus ergasticus (F. et H. Fischer 1892).

\section{LICHOMOLGIDÉS INDÉTERMINÉS}

Un certain nombre de Lichomolgidés ectoparasites ont été signalés sans description et doivent être cités ici.

a. Sur des Mollusques nus, aux Philippines (Semper, 1861, p. 105).

$b$. Sur les branchies de Noumea flava (Eliot), Risbec, en NouvelleCalédonie (Risbec, 1928, p. 34 et 169).

c. Sur les branchies de Gymnodoris ceylonica (Kelaart), en Nouvelle-Calédonie (Risbec, 1928, pp. 34-35).

d. Sur les branchies de Platydoris immonda Risbec, en NouvelleCalédonie (Risbẻc, 1928, p. 34). 
$e$. Sur Nacella mytilina (Helbling), Terre de Feu (Pelseneer, 1903, p. 7).

f. Sur Cavolinia tridentata (Forskål), localité inconnue (Boas, 1886, p. 34 « en Ergasilus-lignende Snyltekrebs »).

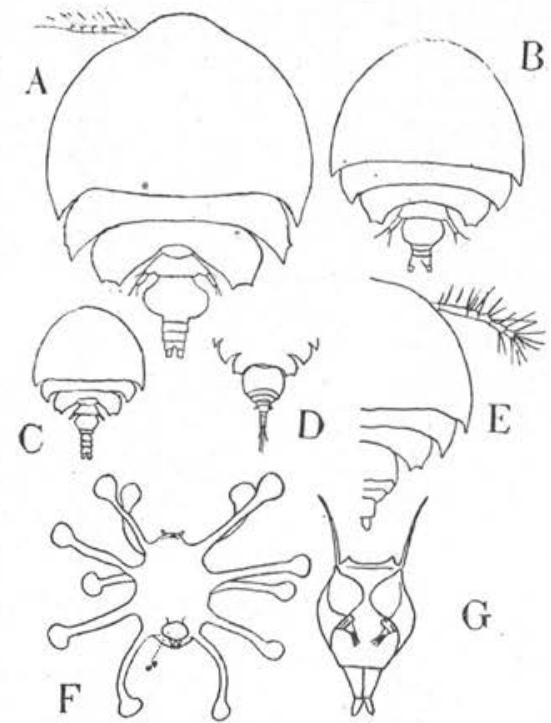

FIG. 10. - A, Lichomolgus (Stellicola) semperi (Kossmann), @, d’après Thompson et A. Scott (1903, pl. XX, fig. 6 " Paralichomolgus longicaudatus »); B, Id. Q, d'après Semper in Kossmann (1877, pl. III, fig. 1); C, Id. O, d'après Stebbing (1900, pl. LXXIV, fig. B, Q "Linckiomolgus caruleus »); D, Id., ᄋ (partie postérieure) et $\sigma^{*}$, in copula, d'après Stebbing $(1900$, pl. LXXIV, fig. B, Ơ "O "Linckiomolgus caruleus »); E, Id., @, d'après Semper in Kossmann (1877, fig. texte, p. 14); F, Copépode énigmatique parasite d'Elysia ornata Pease, Q, d'après Risbec (1930, fig. 4); G, Id., O', d'après Risbec (1930, fig. 6).

\section{Clausiide}

Remarques. — La famille des Clausiidæ — qu'il faut se garder de confondre avec celle des Clausidiidx - a été créée par G. O. Sars (1918, p. 200) pour recevoir Clausia Claparède et Conchocheres G. O. Sars 1918. Il nous semble qu'outre Clausia et Panaietis Stebbing 1900 (= Conchocheres) la famille devrait comprendre Mytilicola Steuer et Trochicola Dollfus (1), probablement aussi Myicola Wright et

(1) Plusieurs des genres de Pelseneer (1928), énigmatiques en l'absence de tout renseignements sur les appendices autres que les antennules ou la $5^{\mathrm{e}}$ patte thoracique, appartiennent peut-être aussi aux Clausiidés. 
Lecanurius Kossmann (fig. 17 B). Il est évident que les Clausiidés sont très voisins des Ergasilidés et doivent être placés tout à côté de ces derniers.

La diagnose provisoire de la famille serait à peu près, en modi-

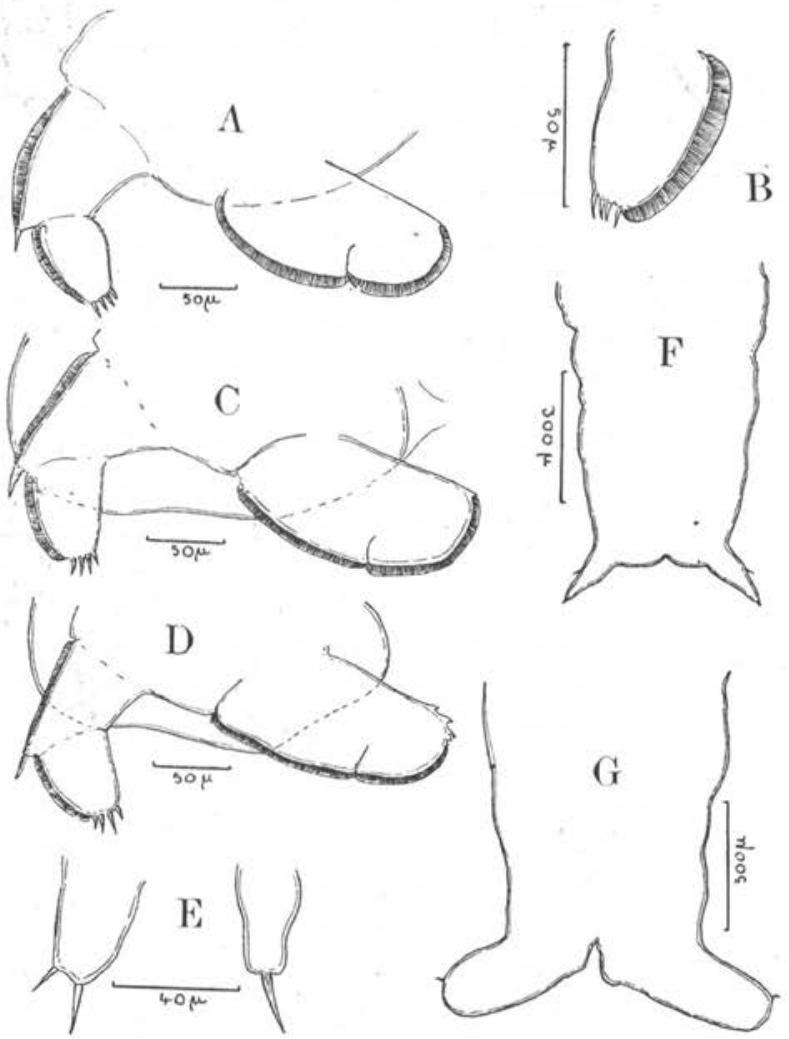

FIG. 11. - A, Trochicola entericus Dollfus, $\odot, 1^{\text {re }}$ patte thoracique ; B, Id., $2^{\circ}$ article de l'exopodite; C, Id., $2^{e}$ patte thoracique; D, Id., $3^{\mathrm{e}}$ patte thoracique ; E, Id., $5^{\text {s }}$ patte thoracique ; F, Id., extrémité postérieure du corps ; G, Mytilicola intestinalis Steuer, Q̊, extrémité postérieure du corps.

fiant légèrement celle de G. O. Sars, 1918 (pp. 200-201) la suivante : Dimorphisme sexuel accentué, le mâle, sans être " pygmée », notablement plus petit que la femelle est immédiatement reconnaissable à la présence d'un appendice supplémentaire (maxillipède). Corps de la femelle plus ou moins allongé, sans démarcation nette entre les divisions antérieure et postérieure, celle-ci étant composée du 
nombre normal de somites ou non distinctement segmentée. Antennules courtes (5-7-articulées), coniques. Antennes courtes, préhensiles, terminées par une griffe. Pièces buccales plus ou moins réduites: mandibules très petites, non connues avec certitude chez Mytilicola et Trochicola; maxillules tuberculiformes, sétigères; maxilles robustes, transversales, à forte musculature basale et à partie apicale
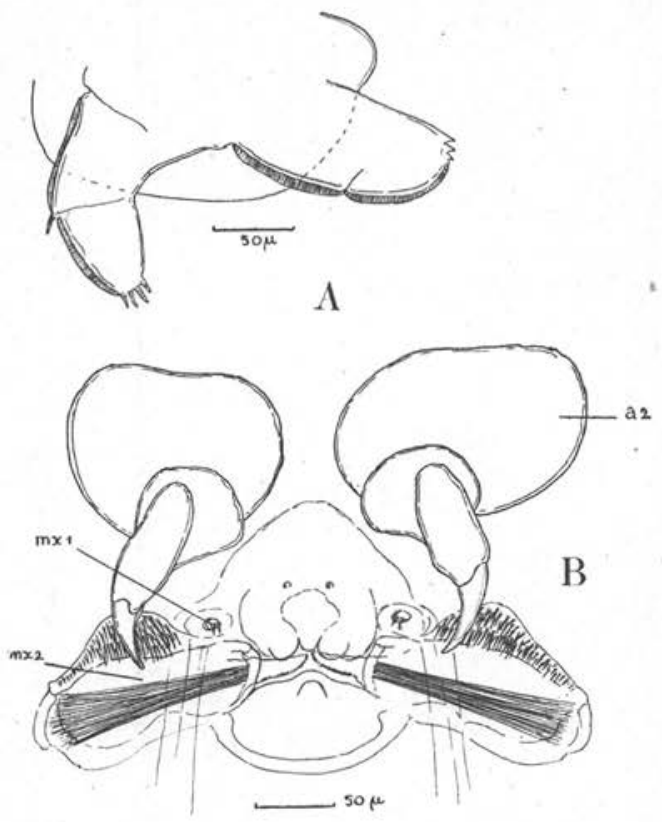

Fig. 12. - A, Trochicola entericus Dollfus, $\bigcirc 4^{\mathrm{e}}$ patte thoracique; $\mathrm{B}, I d$., région buccale.

aiguë, denticulée ou non ; maxillipède absent ou obsolète chez la femelle, présent et sub-chelaté chez le mâle. Pattes thoraciques tantôt développées, à rames 3 -articulées, tantôt réduites, à rames 2-articulées ; $5^{\circ}$ paire présente, généralement 1 -articulée, développée ou obsolète. Deux sacs ovigères, plus ou moins longs, parfois filiformes, contenant des œufs irrégulièrement dispersés.

\section{Panaietis Stebbing 1900.}

\section{Conchocheres G. O. Sars 1918}

Remarques. - Sars ne mentionne pas Panaietis et il est impossible d'échapper à l'impression que les deux genres sont synonymes. 


\section{Panaietis incamerata Stebbing 1900}

Fig. 17 A.

1900. Panaietis incamerata Stebbing, pp. 666-667, pl. LXX, fig. E. Loc. - Panaieti, Archipel de la Louisiade.

Hote. - Gastéropode indéterminé.

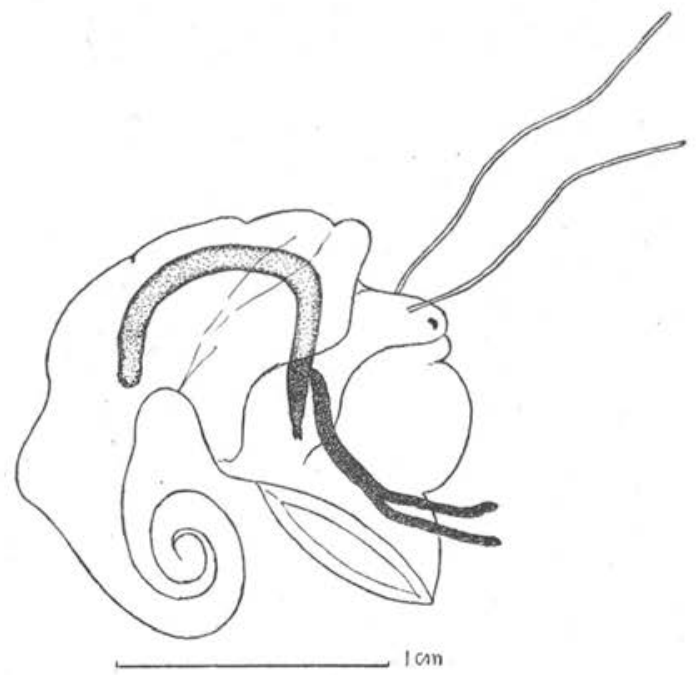

Fig. 13. - Trochicola entericus Dollfus, $\odot$ in silu, d'après un croquis de R.-Ph. Dollfus.

\section{Panaietis malleolata (G. O. Sars 1918)}

Fig. $16 \mathrm{C} ; 17 \mathrm{C}$.

1918. Conchocheres malleolatus G. O. Sars, pp. 202-203, pl. CXII. Loc. - Côte occidentale de Norvège.

Hòte. - Cuspidaria obesa Lovén.

Mytilicola A. Steuer 1902.

Fig. $11 \mathrm{G} ;-14 \mathrm{~A}$.

Mytilicola intestinalis Steuer 1902

1902. Mytilicola intestinalis Steuer, pp. 635-637, 2 fig.

1903. Mytilicola intestinalis Steuer, pp. 1-46, pl. I-V (fig. 1-82).

1907. Mytilicola intestinalis Pesta, pp. 78-98, pl. VI.

1914. Mytilicola intestinalis Dollfus, p. 1531. 
1914. "Lernaea sp. ? " Vayssière, p. 278, pl. XV, fig. 9. 1927. Mytilicola intestinalis Dollfus, p. 121, note 1.

Hôtes. - Mytilus galloprovincialis Lamarck ; Mytilus edulis Linné Loc. - Méditerranée : Trieste, Marseille, Martigues, Banyuls. (Vayssière).
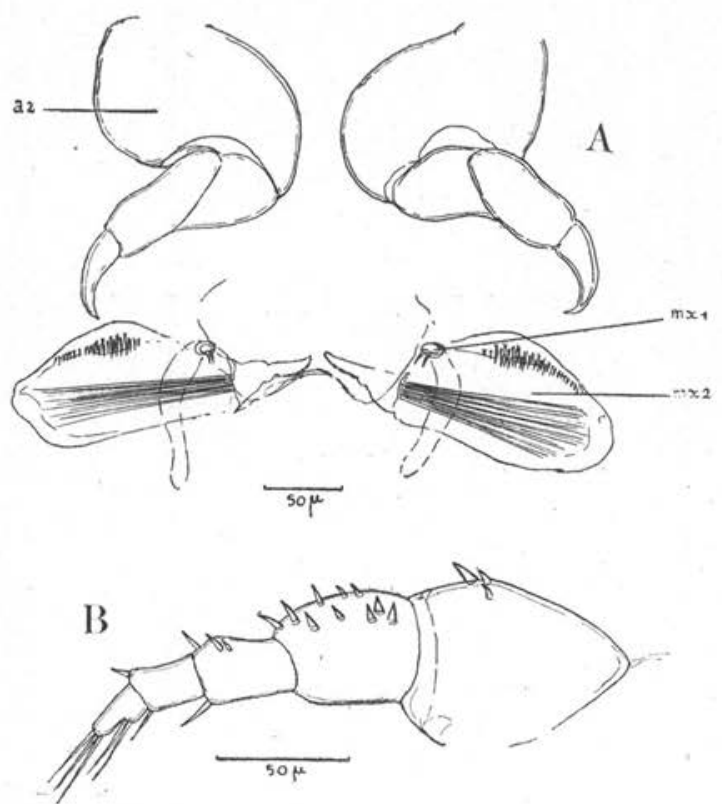

FIg. 14. - A, Mytilicola intestinalis Steuer,, , région buccale; B, Trochicola entericus Dollfus, + antennule.

Trochicola R.-Ph. Dollfus 1914.

Trochicola entericus Dollfus 1914

Fig. $11 \mathrm{~A}-\mathrm{F} ; 12 \mathrm{~A}-\mathrm{B} ; 13 ; 14 \mathrm{~B} ; 15 \mathrm{~A}-\mathrm{B} ; 16 \mathrm{D}$.

1914. Trochicola enterica Dollfus, pp. 1528-1531, 2 fig.

1927. Trochicola enterica Dollfus, p. 121.

Loc. - Manche : St-Vaast-la-Hougue, Roscoff.

Hôtes. - Calliostoma zizyphinum (Linné), var. conuloides (Lamarck), Gibbula cineraria (Linné), Gibbula varia (Linné).

Remarques. - Les notes manuscrites de l'un de nous contiennent quelques renseignements intéressants, encore inédits, sur la fréquence du parasite et un essai d'élevage $a b$ ovo en boîtes de Petri.

«Les Gibbula cineraria, écrit Dollfus, habitant les Fucus serra- 
tus L. dans le voisinage d'un même rocher, peuvent être en majorité parasités, alors que plus loin, les Gibbula ne contiennent pas un seul parasite. » Voici quelques chiffres obtenus à Roscoff ou aux environs : 1 Trochicola sur 40 Gibbula examinés (20. VIII. 1913), 1 Trochicola sur 7 Gibbula (25. VIII. 1913), 1 Trochicola sur 24 Gibbula (1. IX. 1913), 17 Trochicola sur 82 Gibbula (8. IX. 1913), 5 Trochicola sur 25 Gibbula (12. IX. 1913), 2 Trochicola sur 10 Gibbula (27. IX. 1913), 3 Trochicola sur 20 Gibbula (29. IX. 1913), 5 Trochicola sur 70 Gibbula (1. X. 1913), 7 Trochicola sur 90 Gibbula (2. X. 1913), 7 Trochicola sur 65 Gibbula (4. X. 1913).

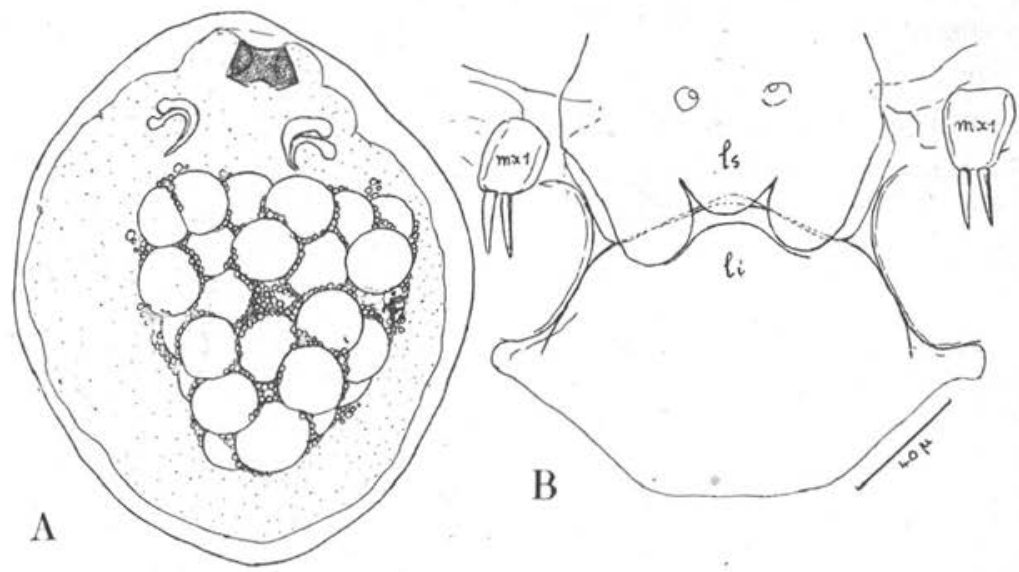

Fig. 15. - A, Trochicola entericus Dollfus, embryon dans l'œuf; B, Id., \&, région buccale montrant les lèvres supérieure et inférieure rapprochées et les maxillules.

Des œufs, contenus encore dans les sacs ovigères et mis en observation ont éclos très peu de temps après $(12$ septembre, $10 \mathrm{~h}$. du matin). Au cours de la journée du lendemain (13 sept.) les nauplius se transformaient en métanauplius et à la fin du troisième jour (14 sept., 22 h. 45) les métanauplius avaient tous passé à l'état de larves copépodites. Les mues se succédèrent rapidement. Le quatrième jour (15 sept., 22 h.) les larves copépodites commencèrent à cesser de nager activement et à tomber sur le fond. Le sixième jour (17 sept.) les larves étaient toutes tombées sur le fond pour mourir bientôt, à une taille de $0,3 \mathrm{~mm}$. L'expérience n'a pu être poussée plus loin.

Les croquis de larves de Trochicola exécutés par Dollfus montrent des copépodites absolument comparables à ceux de Mytilicola (cf. Pesta, 1907). 
D'après des préparations de l'un de nous, nous figurons quelques détails d'organisation de Trochicola pour faciliter la comparaison avec Mytilicola. En fait, jusqu'aux détails de la région buccale ou des pattes thoraciques, il y a entre Trochicola et Mytilicola une ressemblance telle que les caractères différentiels qui subsistent n'apparaissent que tout au plus spécifiques. Pour qui ne connaitrait que la femelle de Trochicola et de Mytilicola aucun doute ne saurait subsister quant à l'évidente congénéricité des deux formes. Jusqu'à présent le mâle de Trochicola est inconnu et l'un de nous a insisté sur le fait que chaque Troque ne contient jamais qu'un parasite, tandis que Mytilus peut en héberger un grand nombre, des deux sexes. Il faut attendre la découverte du mâle de Trochicola pour pouvoir se prononcer quant à la validité du genre, maís il est infiniment improbable que des femelles aussi identiques que Trochicola entericus et Mytilicola intestinalis puissent avoir des mâles extrêmement différents et il est très vraisemblable que les deux genres devront être considérés comme synonymes.

Il faut enfin discuter et tenter de préciser la position systématique de Mytilicola et de Trochicola. Il ne semble guère possible d'affirmer avec Steuer (1903, p. 7) : « Die gesamte äussere Körperform, sowie die Gestalt der Fühler and Mundwerkzeuge weisen auf die Zugehörigkeit der Mytilicola intestinalis zur Familie der Dichelestiina ». Mytilicola (et les genres voisins composant la famille des Clausiidæx) se distingue nettement des Dichelesthiidæx qui ont : un proboscis, un maxillipède développé même chez la femelle, un abdomen très réduit (1) et des cordons ovigères à œufs unisériés.

Il n'y a, chez la femelle de Mytilicola ou de Trochicola que deux paires d'appendices bucaux évidents. Ces deux appendices ont été nommés mandibule et maxilles (Steuer, 1903 ; Pesta, 1906 ; Dollfus, 1914), en admettant que les maxillules manquent. En fait, il n'y a pas de doute en ce qui concerne les maxilles, puisque Steuer a reconnu à leur base le pore excréteur de la glande maxillaire (1903, pl. 2, fig. 9). Il me semble, par contre, extrêmement douteux que le petit moignon tuberculiforme surmonté de 2 épines représente la mandibule. La comparaison des pièces buccales de Mytilicola ou Trochicola avec celles des Ergasilidæ montre que cet appendice est tout à fait analogue - topographiquement et morphologiquement - à celui que Wilson, après Canu (1892), désigne (1911) sous le nom de maxillule : l'argumentation de Wilson (1911,

(1) Wilson $(1922$, p. 19) dit dans la diagnose des Dichelesthiide : « abdomen small and unsegmented, alors qu'il signale un abdomen à plusieurs articles dans les genres Nemesis (p. 58), Eudactylina (p. 65) et Lamproglena (p. 71). 
pp. 282-285) me semble extrêmement sérieuse et la preuve semble faite qu'il s'agit d'appendices vrais $\left(\mathrm{mx}^{1}\right)$ et nullement d'un simple palpe mandibulaire comme l'ont admis certains auteurs (par exemple G.-O. Sars, 1918). Chez Ergasilus (fig. 16 B) ou Conchocheres il existe, en avant de cette maxillule, une paire d'appendices qui sont sans nul doute les mandibules. Au contraire, chez Mytilicola et
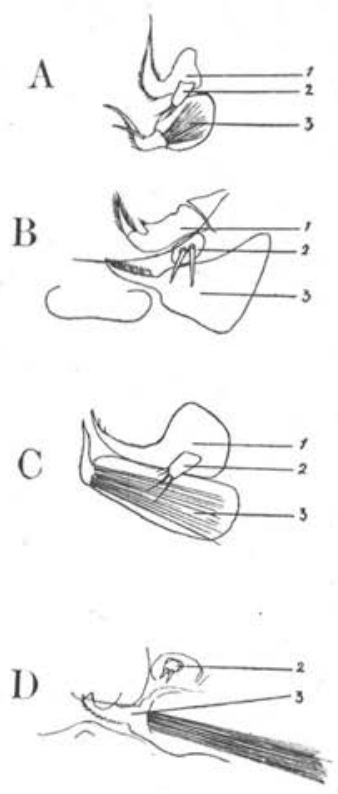

Fíg. 16. - Schéma de la disposition des pièces buccales $(1$, mandibule, 2, maxillule, 3, maxille) chez Lichomolgus sp. (d'après G. O. Sars); B, Id., chez Ergasilus mugilis Vogt (d'après Wilson) ; C, Id., chez Panaietis malleolata (G. O. Sars) (d'après G. O. Sars); D, Id., chez Trochico'a entericus Dollfus (originale).

Trochicola il n'existe, en avant des tubercules sétigères que l'on semble obligé de considérer comme des maxillules, aucun appendice indubitable. Si les mandibules n'ont pas entièrement disparu, elles sont en tous les cas très réduites et si peu visibles que ni chez Mytilicola ni chez Trochicola nous n'avons pu les apercevoir. C'est de l'étude du développement qu'il faut attendre des renseignements précis sur lé devenir des mandibules naupliennes ; malheureusement le travail de Pesta (1906) sur le développement de Mytilicola ne donne pas de renseignements sur les stades les plus intéressants à cet égard, 
Quoi qu'il en soit de l'absence de mandibule chez Mytilicola et Trochicola, on doit, semble-t-il, rapprocher ces deux genres de la famille des Clausiidæx, dans laquelle ils occuperaient une position similaire à celle des formes sans mandibules chez les Ascidicolidæ.

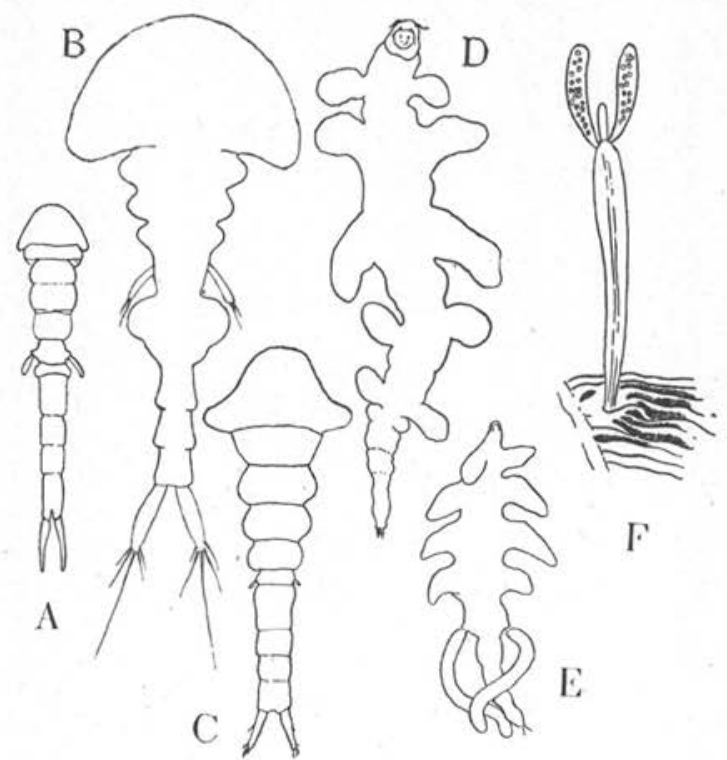

FIG. 17. - A, Panaietis incamerata Stebbing $\odot$, d'après Stebbing (1900, pl. LXX, fig. E); B, Lecanurius intestinalis Kossmann, O, d'après Kossmann (1877, pl. V, fig. 1); C, "Conchocheres malleolalus G. O. Sars ", ᄋ, d'après G. O. Sars (1918, pl. CXII); D, Briarella microcephala Bergh, \&, d'après Bergh (1876, pl. XLIX, fig. 11): E, Briarella sp. Bergh, o, d'après Bergh (1877, pl. LI, fig. 16); F, Ceras-

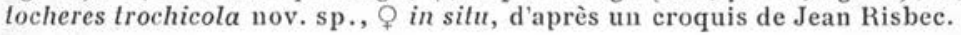

\section{Myicola Wright 1885.}

Remarques. -- Il semble que le genre Myicola, dont la femelle n'a pas de maxillipède, puisse prendre place dans la famille des Clausiidx.

\section{Myicola metisiensis Wright 1885}

1885. Myicola metisiensis Wright, pp. 121-124, pl. III (fig. 1-10). Loc. - Little Metis (Québec, Canada).

Hòte. - Mya arenaria Linné. 


\section{SPLANCHNOTROPHIDA}

Remarques. - La famille a été proposée, pour autant que nous sachions, par Norman et A. Scott, en 1906 (p. 217) qui ont créé le nom sans l'accompagner d'aucune diagnose. Les auteurs du genre Splanchnotrophus, suivis par tous ceux qui ont exprimé un avis sur la position systématique de Splanchnotrophus, rattachent le genre aux Chondracanthidæ: Oakley en 1930 (p. 185), tout en citant Splanchnotrophus parmi les « rejected genera », ajoute que si on le maintient dans les Chondracanthidx il devra constituer au moins la sous-famille des Splanchnotrophinæ et que les renseignements sont insuffisants pour en faire le type d'une famille.

\section{Splanchnotrophus Hancock et Norman 1863.}

Lomanoticola T. et A. Scott 1895.

Remarques. - Il n'est guère possible de distinguer génériquement Lomanoticola de Splanchnotrophus ; toutefois, on peut, très naturellement, diviser le genre en deux sections comprenant, l'une les espèces à bras très grêles, l'autre les espèces à bras courts et trapus; ces deux sous-genres seront Splanchnotrophus sensu stricto et Lomanoticola.

Splanchnotrophus Hancock et Norman 1863.

Sous-genre Splanchnotrophus.

Splanchnotrophus (Splanchnotrophus) gracilis

Hancock et Norman 1863

1863. Splanchnotrophus gracilis Hancock et Norman, pp. 51-54, pl. XV, et XVI, fig. 7-10.

1906. Splanchnotrophus gracilis Norman et A. Scott, p. 217.

Loc. - Manche, côtes d'Irlande.

Hôtes. - Acanthodoris pilosa (Abildgaard in Müller), Idaliella aspersa (Alder et Hancock).

Remarques. - Mme A. Pruvot-Fol a bien voulu nous confier un exemplaire d'Eolidina neapolitana (Delle Chiaje) provenant de Banyuls (Méditerranée) et parasité par un Splanchnotrophus $q$ (fig. 18-19), en très mauvais état malheureusement. Le parasite, qui mesure $3 \mathrm{~mm}$. de long et $8,5 \mathrm{~mm}$. de large, ne semble pas distinct de $S$. gracilis, et on doit se demander si la validité spécifique de $S$. angulatus et de $S$. willemi est bien établie ou s'il ne s'agit en réalité que d'une seule espèce. 


\section{Splanchnotrophus (Splanchnotrophus) willemi}

\section{Canu 1891}

1891. Splanchnotrophus willemi Canu, pp. 435-436.

1894. Splanchnotrophus sp., Pelseneer, p. 147, pl. XV, fig. 126-128.

1896. Splanchnotrophus willemi Hecht, pp. 87-91.

1899. Splanchnotrophus willemi Canu, p. 74.

1906. Splanchnotrophus willemi Pelseneer, note, p. 179.

1927. Splanchnotrophus willemi Cuénot, p. 287.

Loc. - Manche, Golfe de Gascogne (Arcachon).

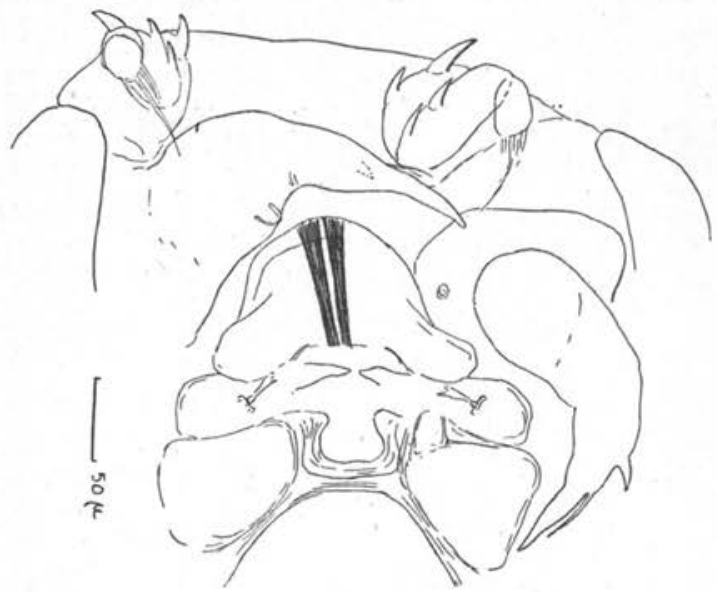

FIG. 18. - Splanchnotrophus sp. [? gracilis Hancock et Norman], parasite d'Eolidina neapolitana (Delle Chiaje), région céphalique.

Hótes. - Facelina longicornis (Montagu), Ancula cristata (Alder). Si la détermination du parasite est exacte, ce dont il n'y a pas lieu de douter, l'espèce parasiterait non seulement le Facelina mais Ancula cristata (cf. Canu 1899 et Pelseneer 1906) et ne saurait être qualifiée de «toujours rigoureusement spécifique»(Cuénot, 1927, p. 287).

Splanchnotrophus (Splanchnotrophus) angulatus Hecht 1893

1893. Splanchnotrophus angulatus Hecht, pp. 15-16, fig. 1.

1896. Splanchnotrophus angulatus Hecht, pp. 87-91, 167, 168, pl. II, fig. 14-16, 18.

Loc. - Manche, Roscoff ; Méditerranée, Toulon.

Hôtes. - Eolidina glauca (Alder et Hancock), Eolidina $[=S p u-$ rilla] neapolitana Delle Chiaje, Aeolidia papillosa (Linné). 


\section{Splanchnotrophus (Splanchnotrophus) sacculatus} O'Donoghue 1924

1924. Splanchnotrophus sacculatus O'Donoghue, pp. 571-573, pl. 30, fig. 65-66.

Loc. - Iles Abrolhos.

Hôte. - Ceratosoma brevicaudatum Abraham.

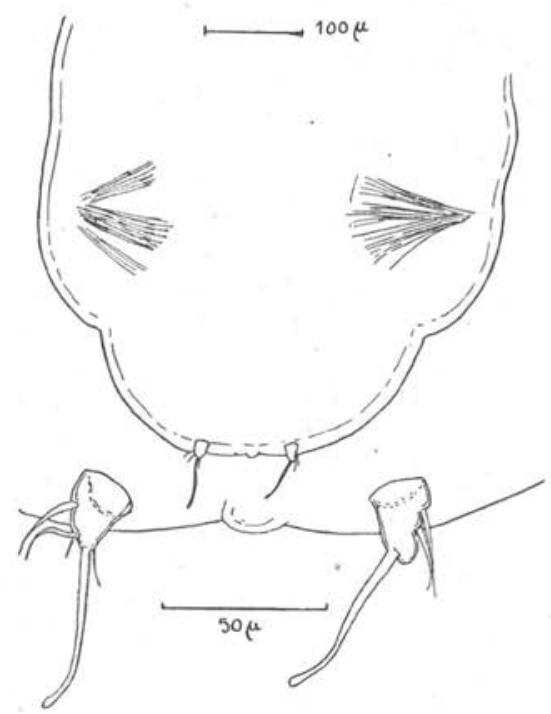

FIG. 19. - Splanchnotrophus sp. (? gracilis Hancock et Norman], parasite d'Eolidina neapolitana (Delle Chiaje), région postérieure du corps.

\section{Splanchnotrophus (Splanchnotrophus) sp.}

1903. Splanchnotrophus sp., Cuénot, pp. 16-17, pl. fig. 5-6. 1927. Splanchnotrophus sp., Cuénot, p. 287, fig. 3.

Loc. - Golfe de Gascogne, Arcachon.

Hôte. - Staurodoris verrucosa (Cuvier).

\section{Sous-genre Lomanoticola.}

Splanchnotrophus (Lomanoticola) brevipes

Hancock et Norman 1863

1863. Splanchnotrophus brevipes Hancock et Norman, pp. 55-59, pl. XVI, fig. 1-6.

1867. Splanchnotrophus brevipes Bergh, pp. 124-125. 
1868. Splanchnotrophus brevipes Bergh, p. 137.

1876. Splanchnotrophus brevipes Bergh, p. 409, note 1 .

1879. Splanchnotrophus brevipes Bergh, p. 568.

1896. Splanchnotrophus brevipes Hecht, pp. 87-91, pl. II, fig. 17.

Loc. - Mer du Nord, Kattegat, Northumberland; Manche, Roscoff.

Hôtes. - Egalvina viridula (Bergh), Coryphella rufibranchialis (Johnston), Idula coronata (Gmelin), Idula pennatifida (Montagu).

\section{Splanchnotrophus (Lomanoticola) insolens}

T. et A. Scott 1895

1879. Ismailia (sic) sp., Bergh, p. 558.

1890. Splanchnotrophus sp., Garstang, p. 426.

1895. Lomanoticola insolens T. et A. Scott, pp. 360-361, pl. XVII, fig. 1-2.

1896. Lomaniticola (sic) insolens Hecht, p. 88.

1901. Ismaïla sp., Vayssière, p. 88.

1903. Splanchnotrophus sp., Vayssière, pp. 99, 102, pl. II, fig. 23. 1905. Lomanoticola insolens Pearson, p. 163.

Loc. - Manche, Plymouth; Irlande, Valentia ; Méditerranée, Marseille, Naples, Banyuls-sur-Mer ( $\sigma^{\star}$ et ${ }^{\circ}$, Mme Pruvot-Fol leg.).

Hôte. - Lomanotus genei Vérany.

\section{Splanchnotrophus sp.}

1879. Splanchnotrophus sp., Bergh, p. 568.

Loc. - Bergen, Norvège.

Hôte. - Favorinus branchialis (Müller).

\section{? Splanchnotrophus sp.}

1910. Splanchnotrophus sp. Eliot, p. 26, note 2.

Loc. - Inconnue (mers tropicales).

Hôte. - «In a tropical Dorid $30 \mathrm{~mm}$. long. »

Remarques. - L'auteur écrit :- « a Splanchnotrophus (or species of an allied genus). »

\section{Ismaïla Bergh 1867.}

Ismaïla monstrosa Bergh 1867

1867. Ismaïla [Ismaila] monstrosa Bergh, pp. 116, 120-124, pl. IV, fig. B. 
1868. Ismaila monstrosa Bergh, p. 137, pl. I, fig. 20-22.

1898. Ismaila monstrosa Bergh, pp. 506-507, 576, pl. 29, fig. 26-30. Loc. - St-Thomas, Antilles ; Tambes, Chili.

Hotes. - Phidiana lynceus Bergh, Archidoris incerta Bergh.

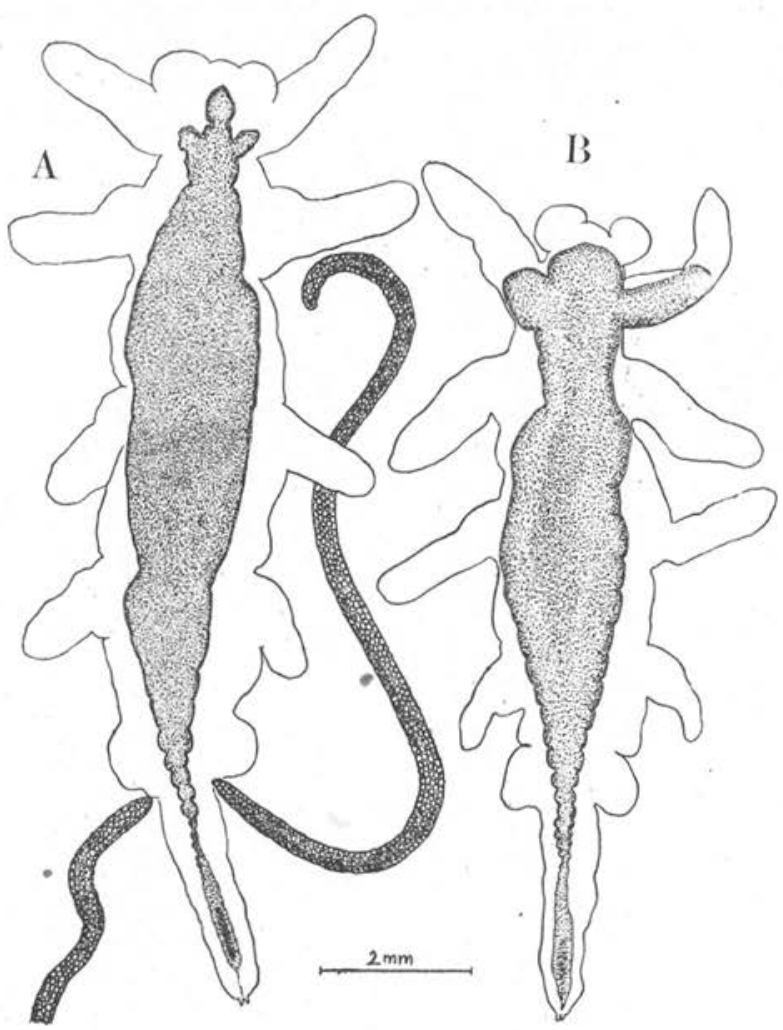

FIG. 20. - A, B, Briarella disphaerocephala nov. sp., ९.

\section{Briarella Bergh 1876.}

Remarques. - Le genre Staurosoma Will 1844, parasite d'Actinies, parait extérieurement très voisin de Briarella, mais est décrit comme dépourvu de pièces buccales (Okada, 1927).

\section{Briarella microcephala Bergh 1876}

Fig. 17 D.

1876. Briarella microcephala Bergh, pp. 409-410, pl. XLIX, fig. 11-13. 
1928. Briarella microce phala Monod, pp. 8, 13-14.

Loc. - Mer Rouge.

Hote. - Ceratosoma trilobatum (Gray).

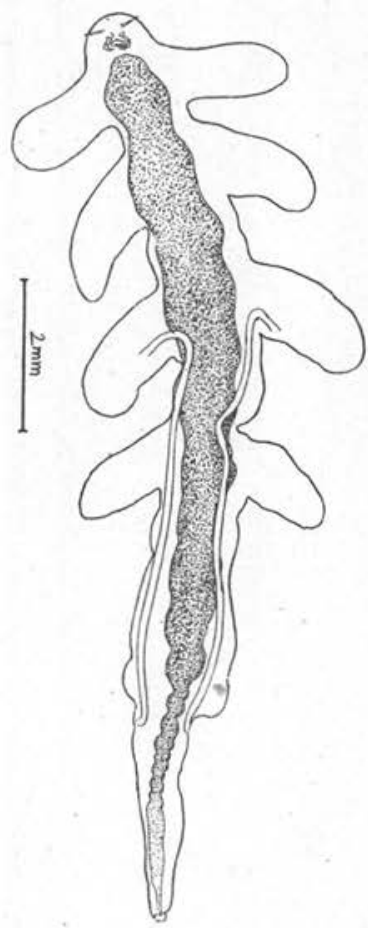

FIG. 21. - Briarella risbeci Monod, $Q$ provenant d'Hexabranchus marginalus (Quoy et Gaimard) [exemplaire inédit non figuré en 1928].

\section{Briarella risbeci Monod 1928}

Fig. $21 ; 24$ E-F ; 26 A

1928. Briarella risbeci Monod [pro parte], pp. 9-15, fig. 5 A-8, 10 [nec fig. 9].

1928. Briarella risbeci Risbec [pro parte], p. 37, fig. $1, \mathrm{n}^{\circ 5} 2-3$ [nec $\mathrm{n}^{\circ} 1$ ] , fig. 1 bis, $\mathrm{n}^{\circ} 4$.

Loc. - Nouvelle-Calédonie.

Hôte. - Hexabranchus marginatus (Quoy et Gaimard).

Les spécimens que l'un de nous a signalés en 1928 sur Platydoris cruenta (Quoy et Gaimard) appartiennent à l'espèce suivante : 
Briarella disphærocephala nov. sp. (1)

Fig. 20 A-B ; 22 A-B ; 23 A-H ; 24 A-D ; 25 ; 26 B-D ; 27

1928. Briarella risbeci Monod [pro parte], pp. 9-15, fig. 9 A-B . [nec, fig. $5 \mathrm{~A}-8,10]$.

1928. Briarella risbeci Risbec [pro parte], p. 37, fig. $1, \mathrm{n}^{\circ} 1$ [nec, $\left.\mathrm{n}^{\circ *} 2-3\right]$.

1929. [Copépode parasite] Risbec, p. 276.

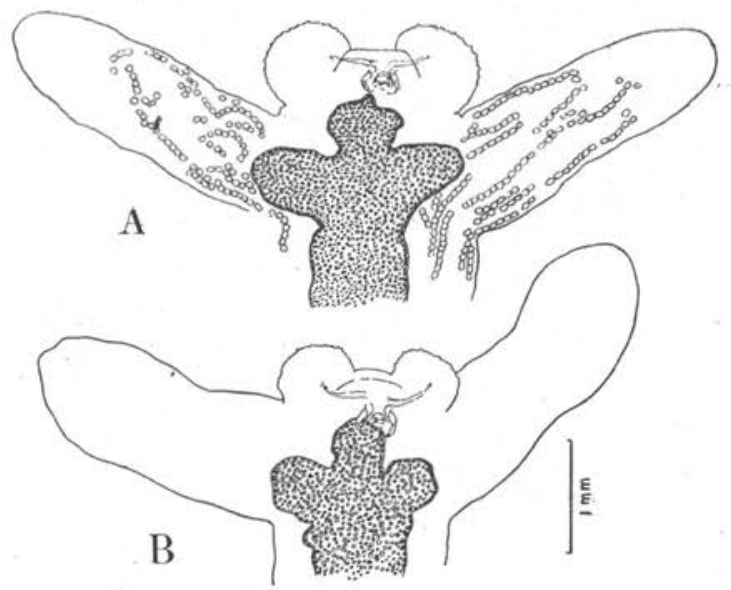

FIG. 22. - A, B, Briarella disphaerocephala nov. sp., \&, région antérieure du corps.

Le matériel examiné en 1928 contenait 5 petits échantillons, assez mal conservés, et provenant de Platydoris cruenta (Quoy et Gaimard). L'un de nous avait rapporté ces échantillons au Briarella risbeci, le décrivant d'après d'excellents exemplaires provenant d'Hexabranchus marginatus (Quoy et Gaimard). Ayant reçu depuis une série de Briarella, en parfait état de conservation et provenant de Centrodoris inframaculata (von Jhering), l'un de nous a pu se convaincre que le parasite de Centrodoris et celui de Platydoris appartenaient à une mème espèce, suffisamment différente du Briarella risbeci d'Hexabranchus pour en être spécifiquement distinguée.

Loc. - Nouméa et Ile Mouac, Nouvelle-Calédonie.

Hôtes. - Platydoris cruenta (Quoy et Gaimard), Centrodoris inframaculata (von Jhering).

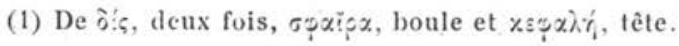

Andi.es de Panasitologie, $\mathrm{T} . \mathrm{X}, \mathrm{N}^{\circ} 2,-1^{\text {er }}$ mars 1932.

12 . 
Matériel utilisé pour la description. - Plusieurs exemplaires, $\sigma^{7} \sigma^{2}$ et $\uparrow \subsetneq$, dans Centrodoris inframaculata (von Jhering), Ile Mouac, Nouvelle-Calédonie, J. Risbec coll.

Description. - Briarella risbeci et B. disphærocephala sont si voisins qu'il suffira, pour caractériser la seconde espèce néocalédonienne, de marquer ce qui la distingue de la première.

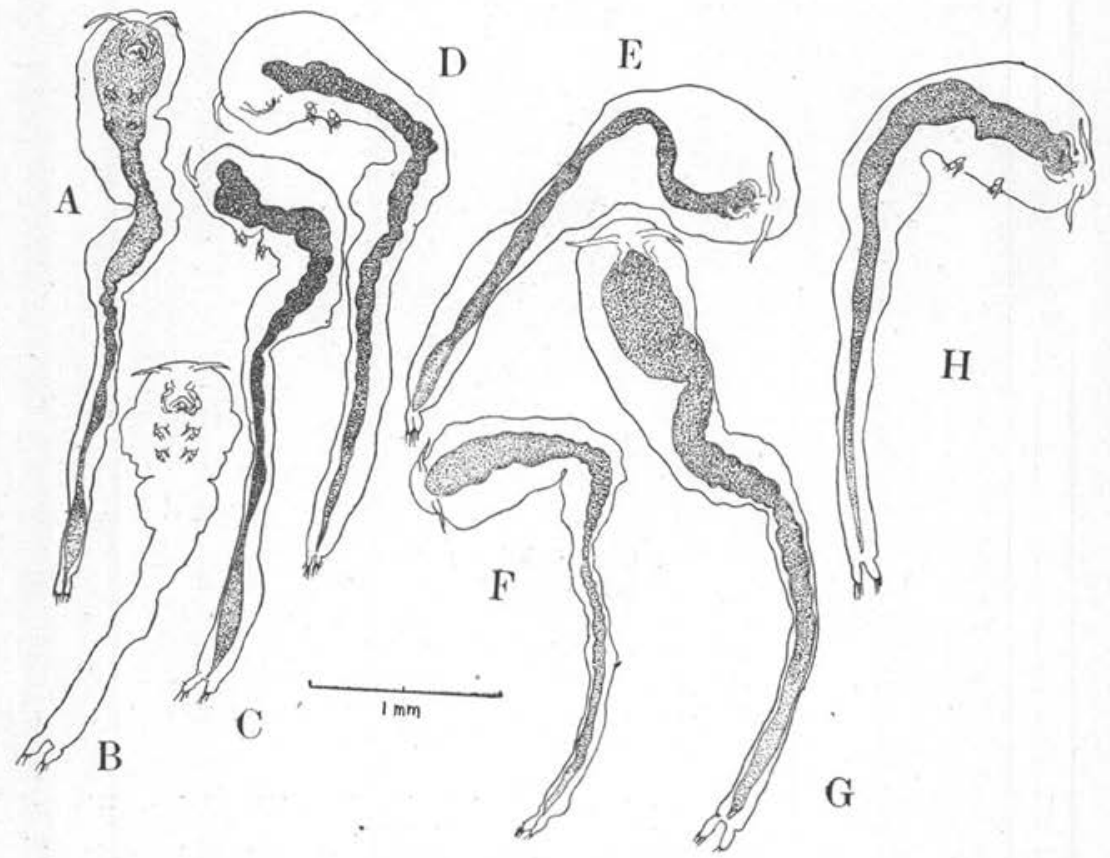

Fıg. 23. - A-H, Briarella disphaerocephala nov. sp., mâles.

\section{B. risbeci :}

Partie antérieure du corps étroitement arrondie.

Lobes latéraux au nombre de 8 $(4+4)$.

Cordons ovigères faisant environ 1 fois $1 / 2$ la longueur totale du corps.

Distance post-génitale égale ou inférieure à la distance séparant l'orifice génital du bord postérieur du 4 e lobe latéral.

\section{B. disphrrocephala:}

Partie antérieure du corps plus ou moins nettement trilobée.

En plus des 8 lobes latéraux principaux $(4+4), 2$ lobes céphaliques arrondis $(1+1)$ et 2 lobes arrondis prégénitaux $(1+1)$.

Cordons ovigères environ aussi longs que le corps.

Distance post-génitale notablement supérieure à la distance séparant l'orifice génital du bord postérieur du 4 e lobe latéral principal. 
Les appendices semblent extrêmement semblables. Cependant la « maxille » de disphærocephala paraît plus grêle que celle de risbeci et possède une petite pointe accessoire, un peu en arrière de l'apex alors que risbeci en serait dépourvue (1). D'autre part, nous n'avons pas observé de « maxillipède » chez disphærocephala.
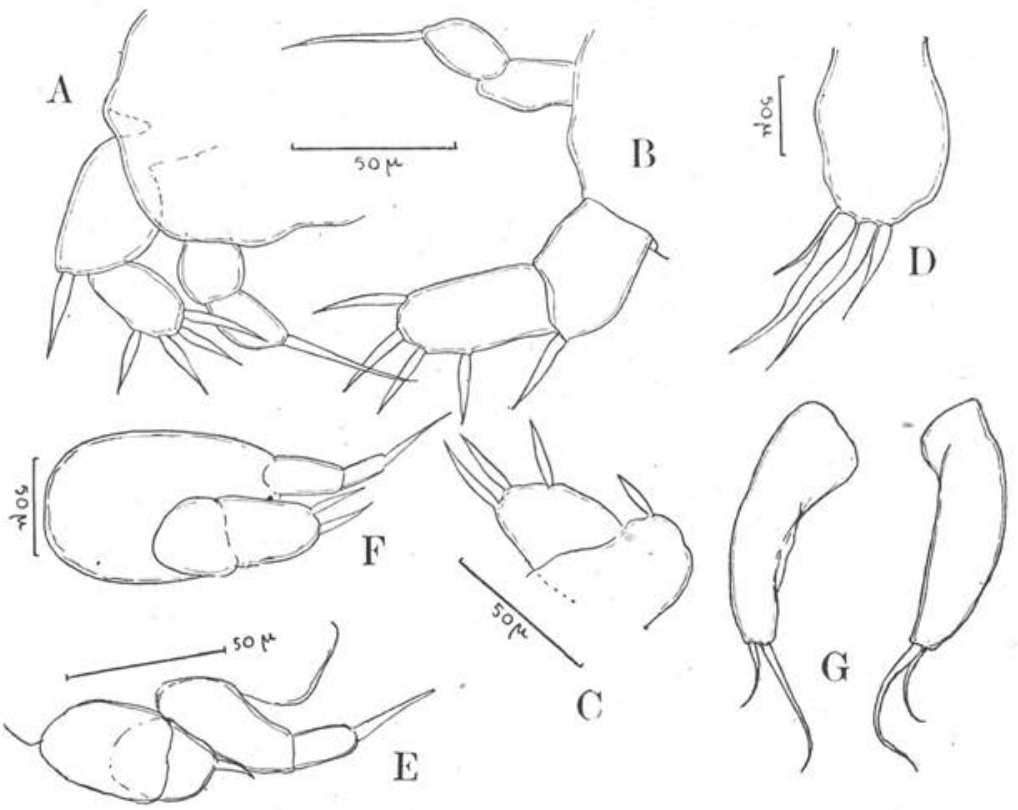

FiG, 24. - A, Briarella disphaerocephala nov. sp., $0^{*}, 1^{\text {ro }}$ patte thoracique; B, Id., $1^{\text {re }}$ patte thoracique; C, Id., exopodite de la $2^{\text {e }}$ patte thoracique; D, Id., branche furcale; E, Briarella risbeci Monod, $ф, 1^{\text {re }}$ patte thoracique; F, Id., $2^{\circ}$ patte thoracique; G, Lichomolgus (Macrochiron) trochi Canu, ${ }^{\circ} 5^{\circ}$ pattes thoraciques: (Le nombre des soies sur l'exopodite des pat'es figurées en $\mathrm{E}$ et $\mathrm{F}$. cst très incertain et la forme des articles modifiée par la mauvaise position des appendices dessinés).

Les tailles sont équivalentes : $16 \mathrm{~mm}$. (risbeci) et $11-13 \mathrm{~mm}$. (disphærocephala). L'aspect général est bien différent dans les deux espèces : nous donnons, pour qu'on en puisse juger, une nouvelle figure de risbeci. On constatera que risbeci est dans l'ensemble plus grêle et ses lobes latéraux plus robustes et plus courts. La tendance à l'allongement de la première paire de lobes latéraux donne à disphærocephala un aspect très particulier : les figures très schématiques données par Risbec (1928, fig. 1) du Briarella de Pla-

(1) La deuxième partie de la première phrase de la p. 10 (Monod, 1928) s'appliquerait seulement à dispharocephala. 
tydoris (B. risbeci) et du Briarella d'Hexabranchus (B. disphrrocephala) soulignent admirablement leurs différences de forme extérieure.

Ajoutons que le tube digestif de disphrrocephala envoie de chaque côté un petit diverticule latéral dans la base du premier lobe latéral principal ce qui n'est pas le cas sur les risbeci examinés par l'un de nous.

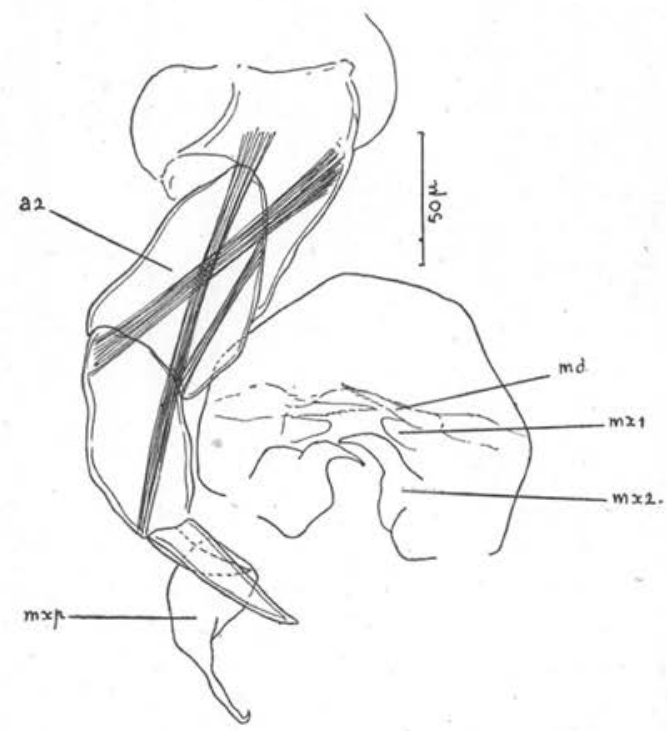

FIG. 25. - Briarella disphaerocephala nov. sp.,

Mélangés aux femelles de disphærocephala se trouvaient 8 mâles, d'autant plus intéressants qu'on ne connaissait jusqu'à présent que deux mâles de Briarella trouvés l'un et l'autre dans Chromodoris elisabethina Bergh et décrits par Bergh (1877, pp. 472-473, pl. LI, fig. $16 \mathrm{~A}-17$ ) qui ne figure ni pièces buccales ni appendices thoraciques.

L'étude des mâles de Briarella disphærocephala a montré qu'ils possédaient toutes les pièces buccales de la femelle, et deux paires d'appendices thoraciques biramés à rames 2-articulées.

Les mâles sont très petits $(2,5-3,5 \mathrm{~mm}$.), plus ou moins vermiformes avec la région antérieure renflée, ce qui leur donne une silhouette claviforme, non sans rapport avec celle des Cumacés typiques. Le tégument est si mince et par conséquent si aisément plissé qu'il en devient impossible de savoir s'il subsiste une seg- 
mentation vraie du corps ou si les plis sont tous accidentels et simplement tégumentaires.

Les pièces buccales sont identiques à celles de la femelle, mais au lieu d'être courts et arrondis les " maxillipèdes 》 sont allongés, composés d'une base dilatée surmontée d'une hampe grêle se terminant en minuscule crochet.

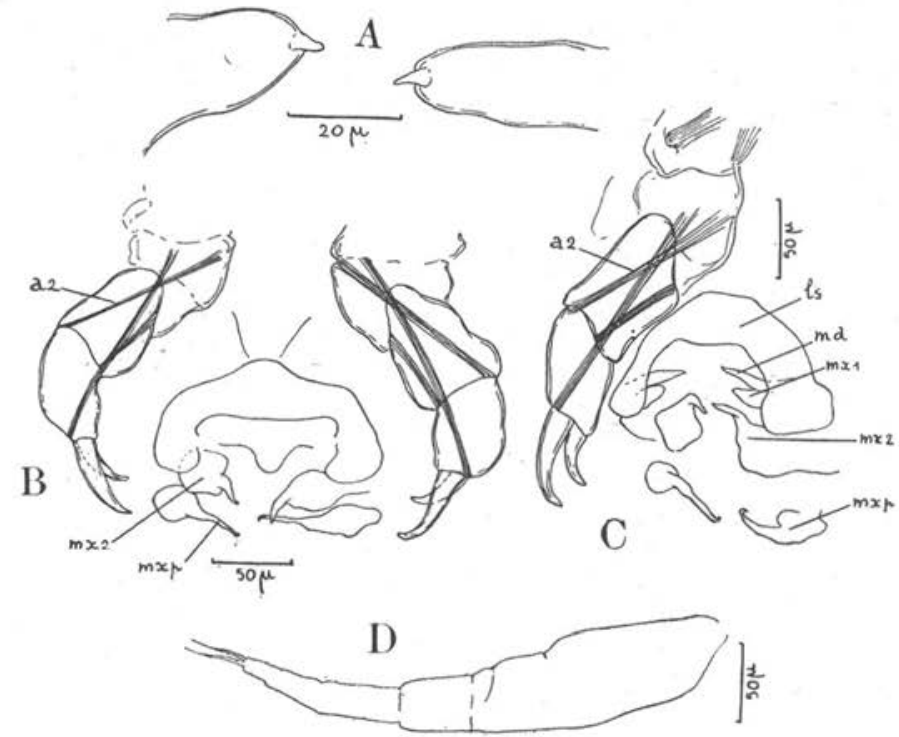

FIG. 26. - A, Briarella risbeci Monod, ९, maxillipède; B, Briarella disphaerocephala nov. sp., O', antennes et pièces buccales; C, Id., antenne et pièces buccales; D, Id., o', antennule.

\section{Briarella sp.}

Fig. 17 E.

1877. Briarella sp. [à la légende de la planche $B$. microcephala] Bergh, pp. 472-473, pl. LI, fig. 16-20.

1878. Briarella sp. Bergh, p. 641 , note 1.

Loc. - Philippines.

Hôtes. - Glossodoris elizabethina (Bergh), Asteronotus bertrana Bergh.

\section{Chondrocarpus Basset-Smith 1903.}

Remarques. - L'auteur décrit et figure très sommairement des Copépodes qui seraient sans nul doute des Briarella s'il ne spécifiait 
que les œufs sont unisériés dans les cordons ovigères et ne les figurait tels. Ce sont des Briarella à cordons ovigères de Dichelesthiidæ: bien qu'il puisse paraitre difficile de confondre des œufs unisériés avec des œufs multisériés, le fait avancé par Bassett-Smith a besoin d'être vérifié pour que la validité générique de Chondrocarpus soit hors de doute. Malheureusement les échantillons ne se trouvent ni au British Museum, ni au Cambridge Museum comme a bien voulu nous le faire savoir le $\mathrm{D}^{\mathrm{r}}$ Isabella Gordon. Il parait extrêmement probable que les Chondrocarpus de Bassett-Smith ne sont que des Briarella mal décrits.

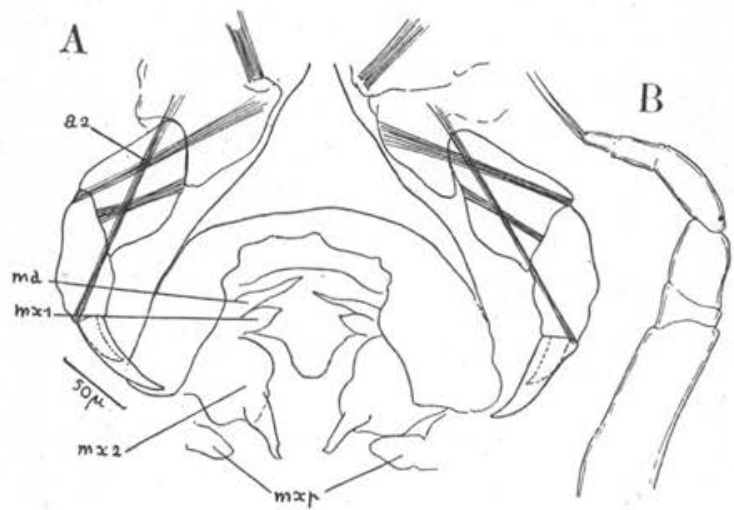

FIG. 27. - A, Briarella disphaerocephala nov, sp., $Q$ antennes et pièces buccales ; $\mathrm{B}, I d$., antennule.

Ghondrocarpus reticulosus Bassett-Smith 1903

1903. Chondrocarpus reticulosus Bassett-Smith, page 105, fig. 11 A.-G.

Loc. - Zanzibar.

Hôte. - "large Pleurobranchid ».

\section{Ghondrocarpus sp.}

1903. Chondrocarpus sp., Bassett-Smith, pp. 105-106, fig. 11, H. Loc. - Zanzibar.

Hóte. - \& Pleurobranchid». 
Caligiformes Oakley 1930 (= Caligoida G. O. Sars 1904).

\section{Caligid $Æ$}

Anchicaligus Stebbing 1900.

Anchicaligus nautili (Willey) Stebbing 1900

1887. « tad-pole like creatures » Smith, p. 226.

1896. Caligus nautili Willey, p. 145, note 2 [nomen nudum].

1900. Anchicaligus nautili Stebbing, pp. 668-670, 686, pl. LXXI.

Loc. - New-Britain (Nouvelle-Guinée).

Hôte. - Nautilus pompilius Linné, Nautilus macromphalus Sowerby.

\section{LERNAIDI (1)}

Pennella Oken 1815.

Pennella varians Steenstrup et Lütken 1861

1877 ? Penella varians Wierzejski, p. 563, pl. 32-33.

Loc. - Trieste.

Hòtes. - Moschites moschatus (Lamarck), Loligo loligo (Linné), Sepia officinalis Linné.

Cerastocheres (2) nov. gen.

Femelle. Corps vermiforme, cylindrique. Céphalothorax avec une paire de cornes latérales coniques et une petite saillie dorsale impaire. Cou rétréci passant insensiblement au tronc. Corps rétréci au niveau des orifices génitaux, l'abdomen étant notablement plus étroit que le tronc, cylindrique, digitiforme, postérieurement arrondi et portant une paire de très petites branches furcales glabres. Sacs ovigères allongés-fusiformes ; œufs multisériés. Deux paires d'antennes, l'antérieure grêle et cylindroïde, sétigère, la postérieure courte et trapue, glabre. Pièces buccales non observées. Au moins trois paires de pattes thoraciques, biramées, à rames $1-\alpha \%$ pị digitiformes, glabres. - Mâle inconnu.

Cette diagnose est essentiellement provisoire, un seul exemplaire (en médiocre état) d'un seul sexe étant connu jusqu'ici.

(1) Voir aussi plus loin p. $180 \mathrm{~d}$.

(2) Cerastocheres, de $x \varepsilon p \dot{\alpha} \sigma \tau \tau_{5}$, , cornu et $\dot{\alpha} \% \eta_{i} \eta_{1}$, importun, douloureux. 


\section{Gerastocheres trochicola (1) nov. sp.}

Fig. $17 \mathrm{~F} ; 28 \mathrm{~A}-\mathrm{B} ; 29$

Loc. - 1 exemplaire ( () sur la branchie de Trochus (Tectus) obeliscus Gmelin (E. Lamy det., d'après une aquarelle de J. Risbec), Nouméa, Nouvelle-Calédonie, J. Risbec coll.

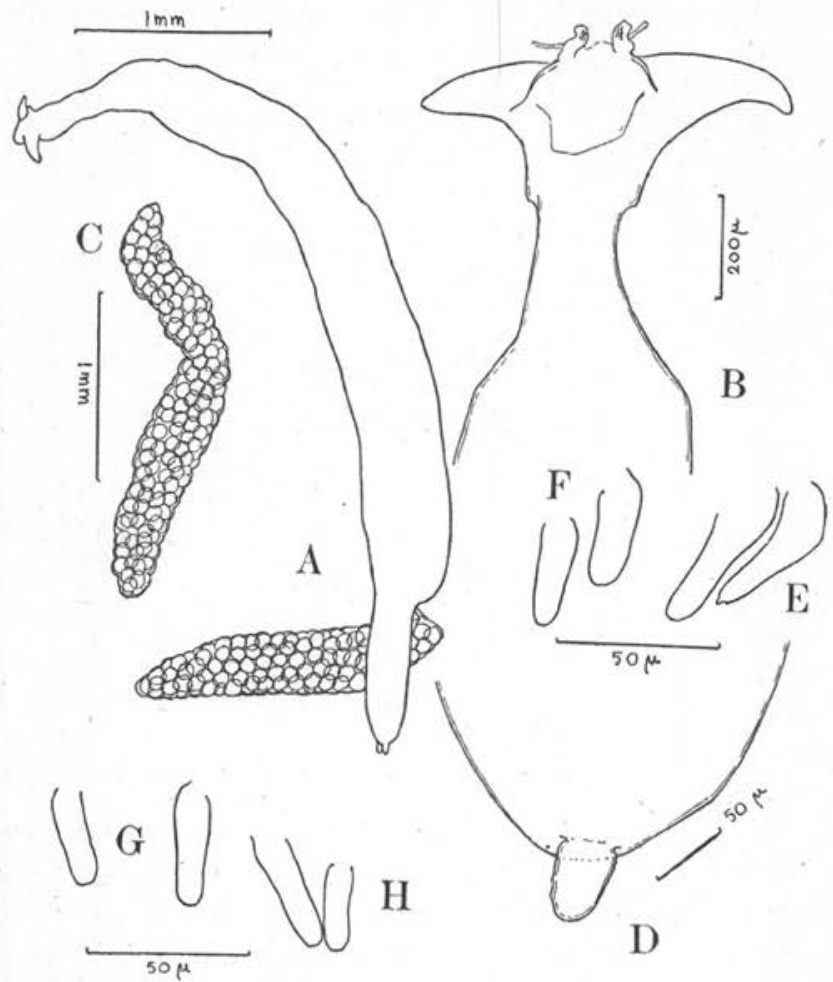

Fig. 28. - A, Cerastocheres trochicola nov. gen. nov. sp., \&, type; B, Id., partie antérieure du corps; C, Id., sac ovigère (le second, celui qui n'est pas représenté en A); D, Id., extrémité postérieure du corps; E, Id., 1re patte thoracique; $\mathrm{F}, \mathrm{G}, \mathrm{Id} ., 2^{\mathrm{e}}$ patte thoracique $\mathrm{H}, \mathrm{Id} ., 3^{\mathrm{e}}$ patte thoracique.

Le Copépode était engagé dans les tissus de l'hôte, le céphalothorax invisible in situ, seules les parties postérieures au cou étant extérieures et libres. C'est une fixation identique à celle de beaucoup de Lernæidæ. "Le Copépode est fixé à la base des feuillets branchiaux qui se trouvent déformés dans la région correspondante. Sa lon-

(1) Trorhicola de Trochus, nom générique de l'hô:e et incola, habilant. 
gueur, relativement considérable, atteignait à peu près la moitié de la longueur totale de la branchie de son hôte. 》(J. Risbec, in litt.).

Description. - Les caractères essentiels ont été signalés dans l'essai de diagnose générique. Il est spécialement regrettable que les appendices céphaliques n'aient pu être analysés de façon satisfaisante. L'antennule, cylindroïde, pauci-articulée, et sétigère ressemble, par exemple, à celle de Lernæa. L'antenne est, sur l'échantillon que j'ai sous les yeux, courte, trapue, et tronquée à son extrémité distale : il n'y a pas d'apparence de crochet. Les deux antennes étant semblables il est peu probable qu'elles aient été l'une et l'autre endommagées de façon absolument identique, ni que le crochet, s'il a existé, ait pu disparaitre de chaque côté sans laisser de trace.

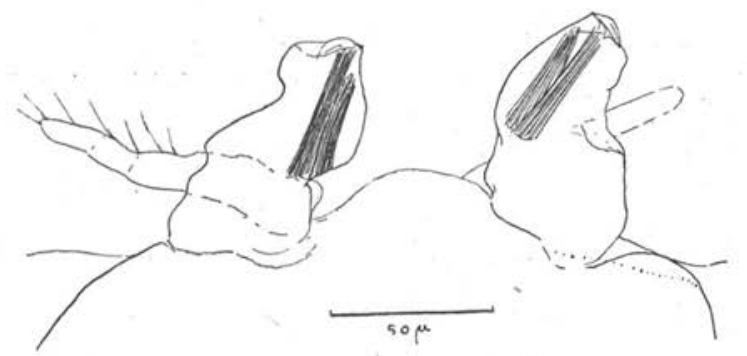

Fí. 29. - Cerastocheres trochicola nov. gen. nov. sp., @, région frontale du spécimen unique.

Quant aux pièces buccales il m'a été impossible d'en découvrir le moindre vestige indubitable. Il existe bien une sorte de cadre buccal, mais ce cadre est vide. La partie médiane du bord frontal étant manifestement abìmée, il est vraisemblable que la face ventrale de la tête a pu l'être également : dans ce cas les pièces buccales auraient été simplement arrachées.

Nous n'avons vu distinctement que 3 paires d'appendices thoraciques mais : $1^{\circ}$ comme la $1^{\text {re }}$ paire observée est déjà assez reculée, placée sur le tronc, il n'est pas du tout invraisemblable que cette paire soit en fait la $2^{\circ}$, la $1^{\text {ro }}$ étant quelque part sur le cou ou à la limite postérieure du céphalothorax ; $2^{\circ}$ il existe peut-être un rudiment d'appendice en avant des orifices génitaux. Taille : ca. 4,5 mm.

Remarques. - La position systématique d'un Copépode aussi mal connu, ne peut être que passablement douteuse. Il semble cependant difficile d'échapper au sentiment qu'il s'agit d'un genre appartenant à la famille des Lernæidæ et à la sous-famille des Lernæinæ. Il est indubitable que dans le mode de fixation, la forme du corps, 
la morphologie du céphalothorax, de l'abdomen, des sacs ovigères, etc... les points de ressemblance ne manquent pas entre Lernæa (Lernæocera auctorum) et Cerastocheres. Ce serait le premier Lernæidé parasite, à l'état adulte, sur un Mollusque.

\section{GENERA INCERTA SEDIS ET NOMINA NUDA}

Paclabius Kossmann 1877.

\section{Paclabius tumidus Kossmann 1877}

1877. Paclabius tumidus Kossmann, pp. 23-24, pl. VI, fig. 1-10.

Loc. - Bohol, Philippines.

Hôte. - Tridacna sp.

Remarques. - La position systématique du genre est incertaine. Les pièces buccales sont celles d'un Lichomolgidé, tandis que la forme externe rappelle à la fois les Clausiidés (Conchocheres) ou les Splanchnotrophidés (Briarella).

\section{Leptinogaster Pelseneer 1928.}

Leptinogaster pholadis Pelseneer 1928

1928. Leptinogaster pholadis Pelseneer, pp. 37-39, fig. 2. Loc. - Naples.

Hôte. - Pholas dactylus. Linné.

Remarques. - Pièces buccales non décrites.

\section{Tococheres Pelseneer 1928}

Tococheres cylindraceus Pelseneer 1928

1928. Tococheres cylindraceus Pelseneer, p. 37, fig. 1.

Loc. - Manche, Roscoff.

Hôte. - Loripes lacteus (Linné).

Remarques. - Pièces buccales non décrites.

\section{Strongylopleura Pelseneer 1928.}

Strongylopleura histrio Pelseneer 1928

1928. Strongylopleura histrio Pelseneer, pp. 39-40, fig. 3. Loc. - Manche, Boulogne.

Hote. - Syndesmya alba (W. Wood).

Remarques. - Pièces buccales non décrites. 
Ameristocheres Pelseneer 1928.

Ameristocheres inermis Pelseneer 1928

1928. Ameristocheres inermis Pelseneer, pp. 41-42, fig. 5.

Loc. - Naples.

Hóte. - Doridium membranaceum Meckel.

Remarques. - L'espèce serait entièrement dépourvue d'appendices ( pas d'appendices céphalothoraciques ni abdominaux», p. 42) ce qui est bien peu vraisemblable et aurait besoin d'être vérifié.

\section{Ischnurella Pelseneer 1928.}

\section{Ischnurella barneæ Pelseneer 1928}

1928. Ischnurella barneæ Pelseneer, pp. 40-41, fig. 4 .

Loc. -- Manche, Wimereux.

Hóte. - Pholas (Barnea) candida Linné.

Remarques. - Pièces buccales non décrites.

\section{Gopépode parasite}

(Fig. 10 F-G)

1930. [Copépode parasite] Risbec, pp. 661-663, fig. 2, $\mathrm{n}^{\circ \mathrm{s}} 3-6$.

Loc. - Nouvelle-Calédonie.

Hote. - Elysia ornata Pease.

Remarques. - Cet extraordinaire animal a un corps ovalaire et 6 paires de prolongements latéraux grêles terminés par une dilatation terminale globuleuse. Le descripteur figure un mâle nain d'un aspect surprenant. Cette forme intéressante mériterait d'être examinée par un spécialiste. L'aspect extérieur de la femelle rapproche celle-ci des Splanchnotrophus.

\section{Copépode parasite}

1911. [Copépode parasite] Pelseneer, p. 3.

1928. [Copépode parasite] Pelseneer, pp. 33, 46.

Loc. -- Océan Indien.

Hóte. - Pandora elongata Carpenter. 


\section{Gopépode parasite}

1911. [Copépode parasite] Pelseneer, p. 3.

1928. [Copépode parasite] Pelseneer, pp. 33, 46.

Loc. - Océan Indien.

Hôte. - Anatina subrostrata Lamarck.

\section{Gopépode parasite}

1928. [Copépode parasite] Pelseneer, pp. 33, 46.

Loc. - Manche.

Hôte. - Pholas (Zirfaea) crispata Linné.

\section{Gopépode parasite}

1928. [Copépode parasite] Pelseneer, pp. 33, 46.

Loc. - Manche.

Hôte. - Ostraea edulis Linné.

\section{Gopépode parasite}

1911. [Copépode parasite] Pelseneer, p. 3.

1928. [Copépode parasite] Pelseneer, p. 33.

Loc. - Océan Indien.

Hóte. - Pinna sp.

\section{NOMENCLATOR HOSPITUM}

Dans les tableaux des pages suivantes les hôtes sont cités par ordre systématique.

Depuis l'impression de ces tableaux, plusieurs références nouvelles sont venues à notre connaissance et n'ont pu y être ajoutées à leur place respective :

a. Lichomolgus agilis (Leydig), signalẻ par Tchang Si (1931, p. 120, 180-181) sous le nom de « $L$. doridicola (Leydig) » à la surface du corps de Doridopsis limbata (Cuvier) à Toulon (Var).

b. Splanchnotrophus angulatus Hecht, signalé par Tchang Si (1931, p. 132, 180-181) chez Spurilla neapolitana (D. Ch.) [corps dans cavité générale, sacs ovigères externes] à Toulon (Var).

c. Splanchnotrophus insolens (T. et A. 'Scott) chez Lomanotus Genei Vérany à Banyuls-sur-Mer (Pyrénées-Orientales).

d. Larves de Lernæidse [? Penella ou Lernæocera ?]. - Cinq spécimens à des stades copépodites dans la cavité palléale et sur les branchies de Janthina exigua. Lamarek [Prosobranchiata Plenoglossa]. Localité inconnue. 


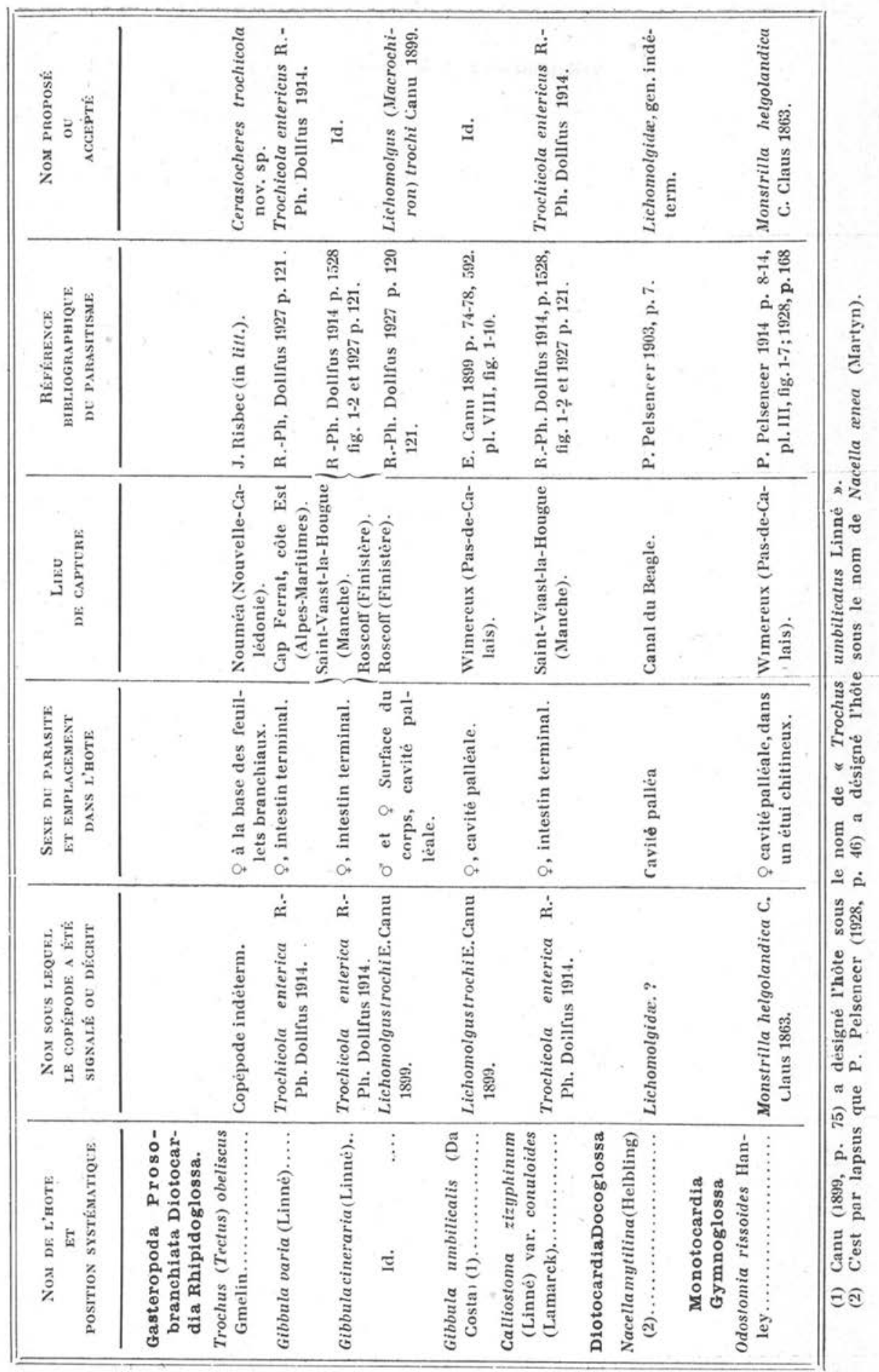




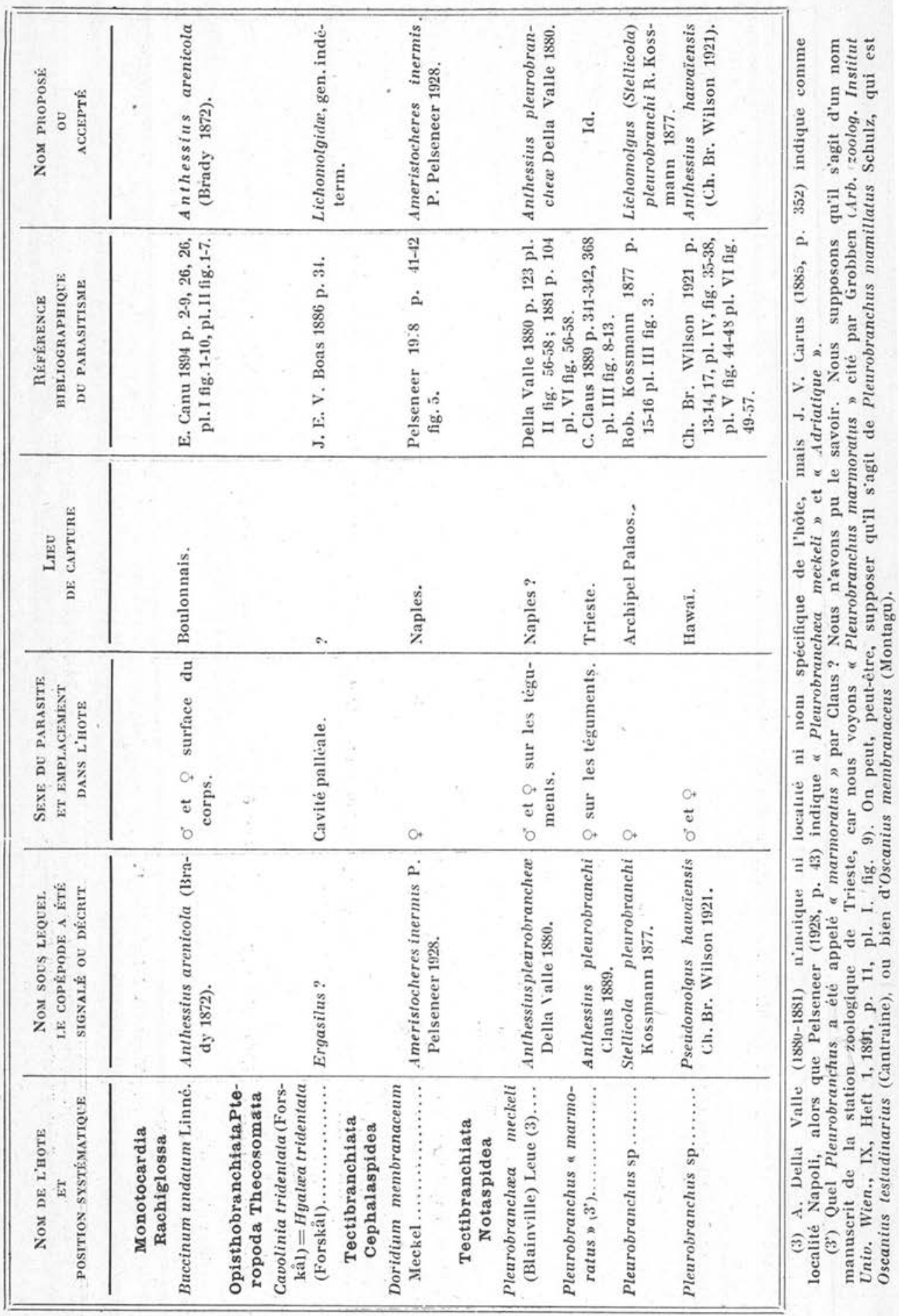




\begin{tabular}{|c|c|c|c|c|c|c|c|c|}
\hline 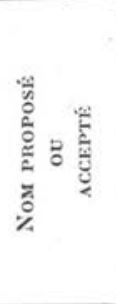 & 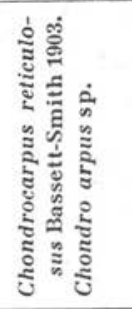 & 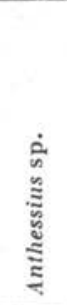 & 竎 & 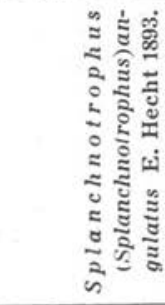 & 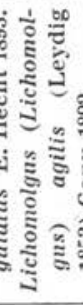 & : & 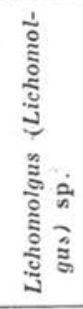 & 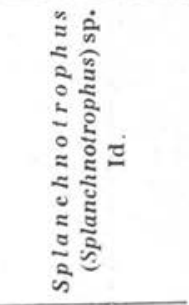 \\
\hline 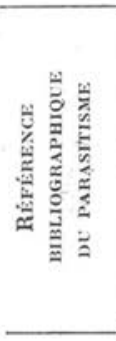 & 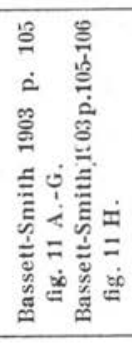 & 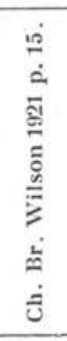 & 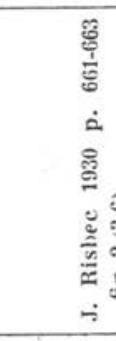 & 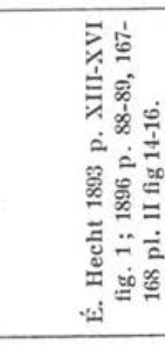 & 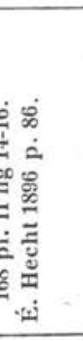 & 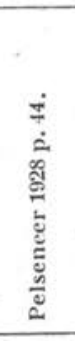 & 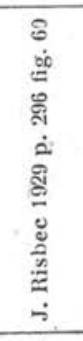 & 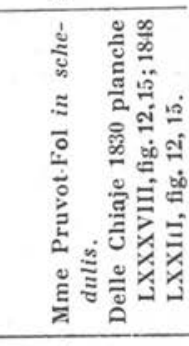 \\
\hline 离 & 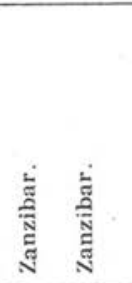 & 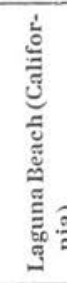 & 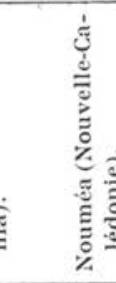 & 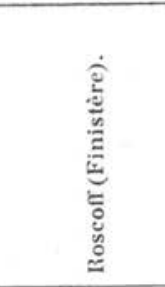 & 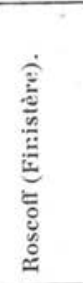 & & 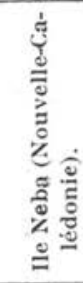 & 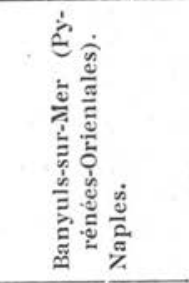 \\
\hline 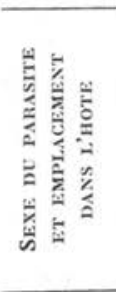 & 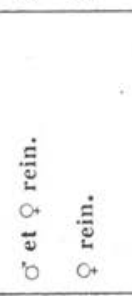 & ct & 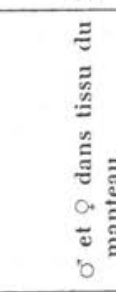 & 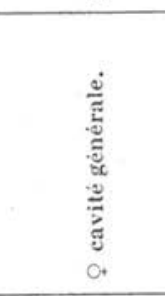 & 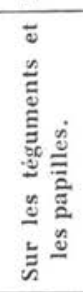 & & 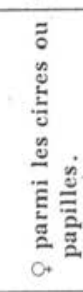 & 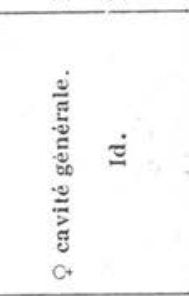 \\
\hline 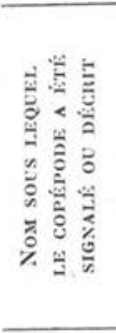 & 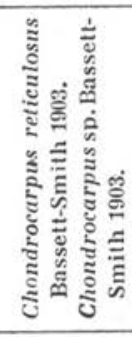 & 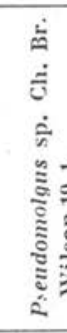 & 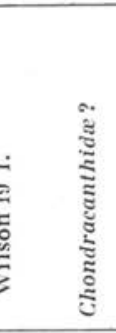 & 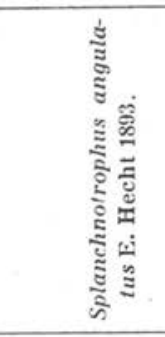 & 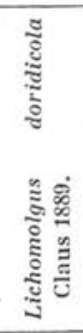 & 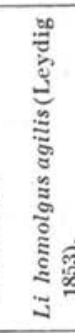 & 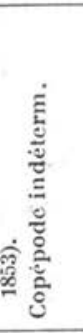 & 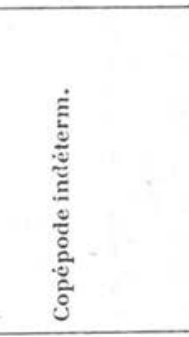 \\
\hline 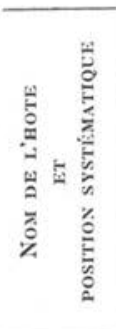 & 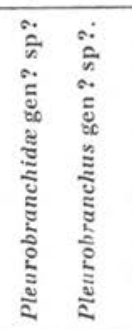 & 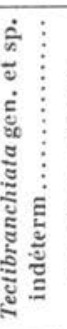 & 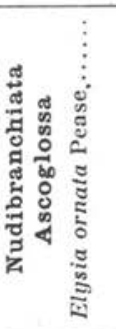 & 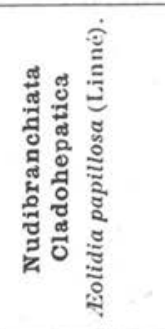 & $\dot{\Xi}$ & $\begin{array}{l} \\
\vdots \\
\dot{\Xi}\end{array}$ & 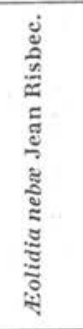 & 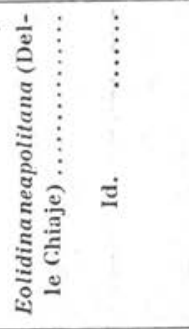 \\
\hline
\end{tabular}




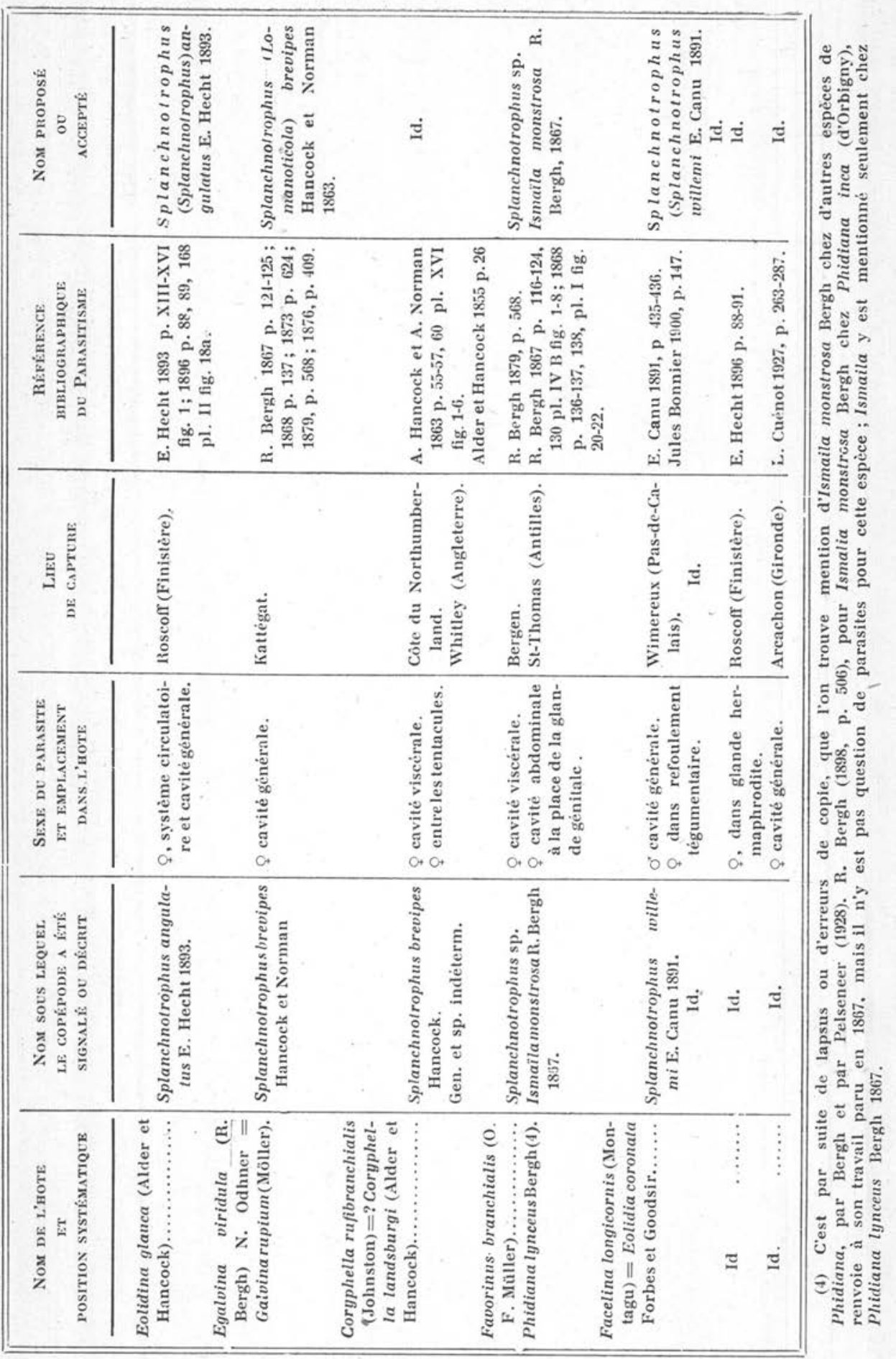




\begin{tabular}{|c|c|c|c|c|c|c|c|c|c|c|c|}
\hline 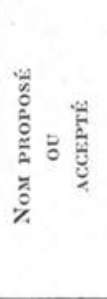 & $\Xi$ & 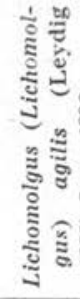 & & $\grave{\Xi}$ & $\Xi$ & & $\dot{\Xi}$ & & & ஹ் & $\dot{\Xi}$ \\
\hline 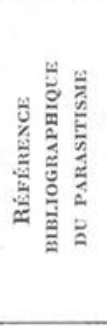 & 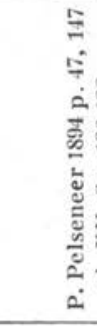 & 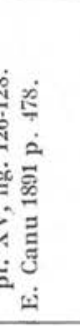 & 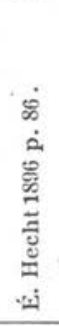 & 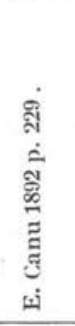 & 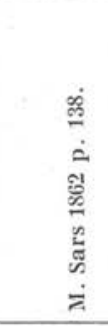 & 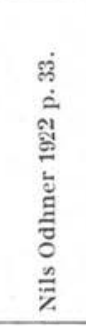 & 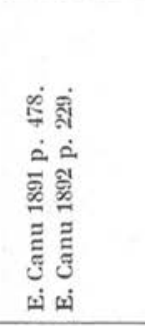 & 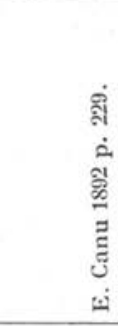 & : & 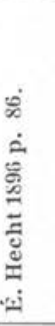 & 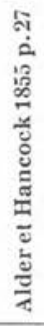 \\
\hline$\frac{\frac{\pi}{2}}{3}$ & 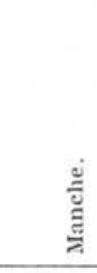 & 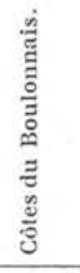 & 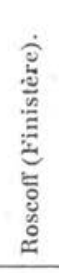 & 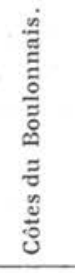 & 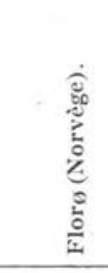 & 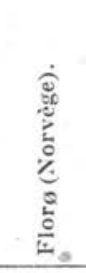 & 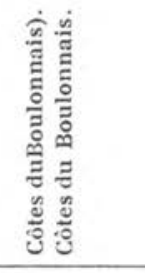 & 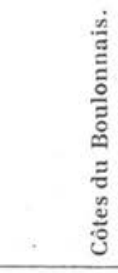 & 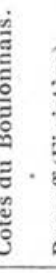 & 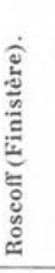 & 范 \\
\hline 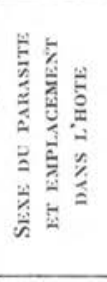 & 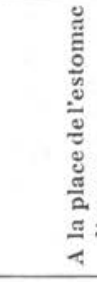 & 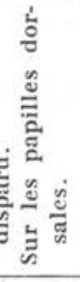 & 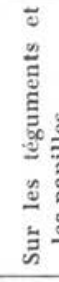 & 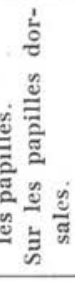 & 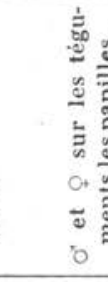 & & 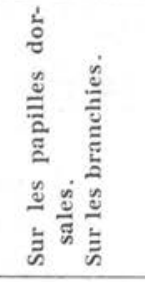 & 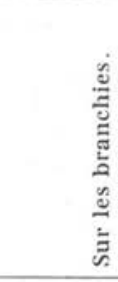 & 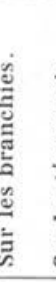 & 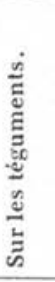 & 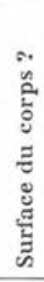 \\
\hline 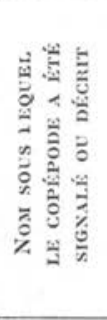 & 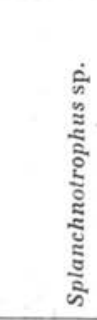 & 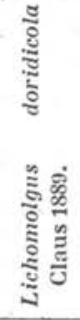 & $\begin{array}{l}\vdots \\
\vdots \\
\\
\vdots\end{array}$ & 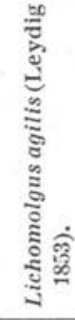 & 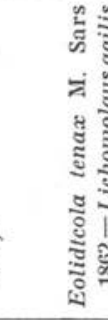 & 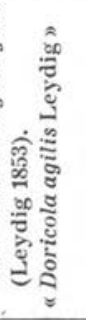 & 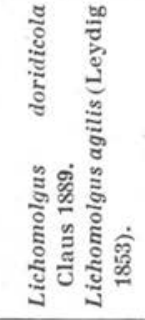 & 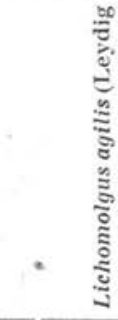 & 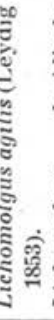 & 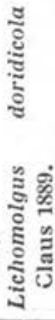 & 焉 \\
\hline 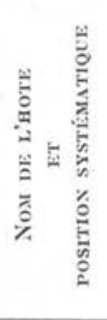 & 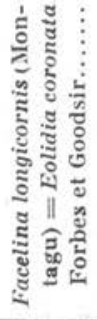 & $\vdots \vdots$ & $\Xi$ & $\vdots$ & 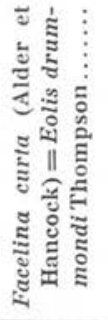 & 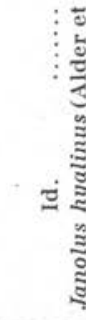 & 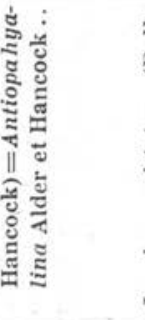 & 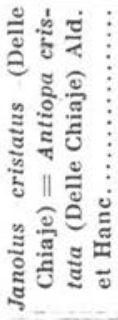 & 迅 & $\vdots$ & $\Xi$ \\
\hline
\end{tabular}

Annales de Parastrologie, T. $\mathrm{X}, \mathrm{N}^{\circ} 2 .-1^{\mathrm{er}} \operatorname{mars} 1932$. 


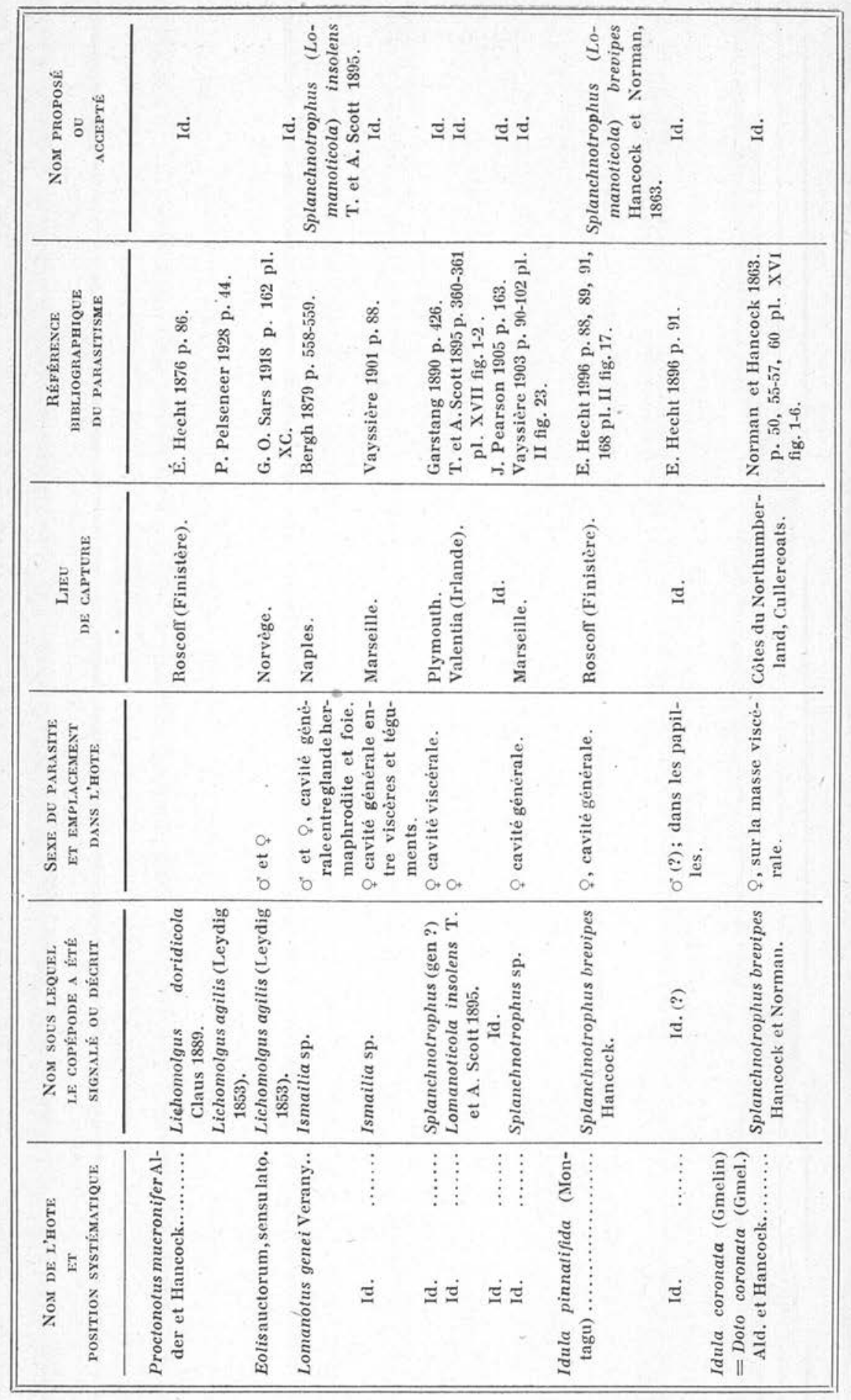




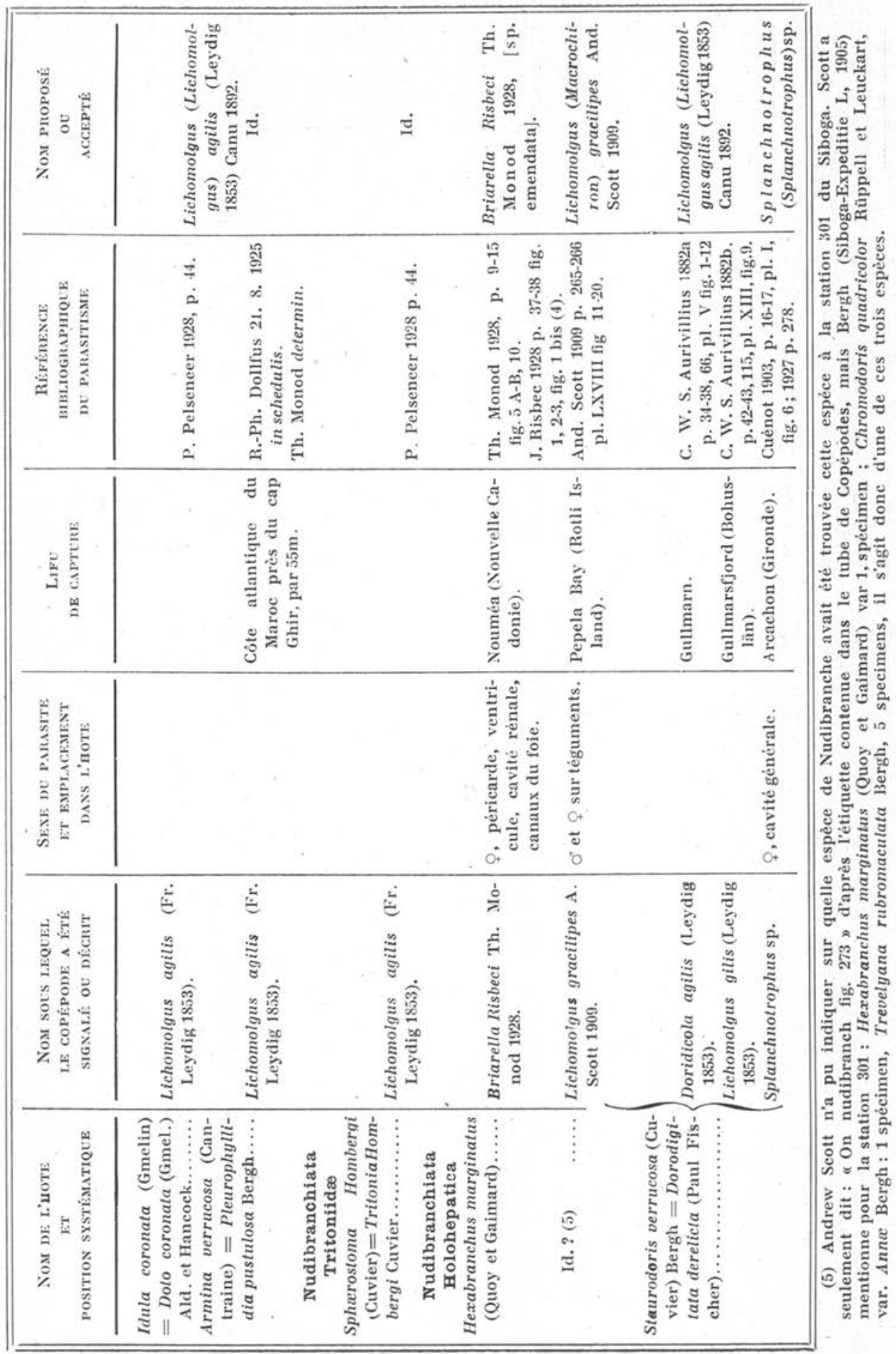




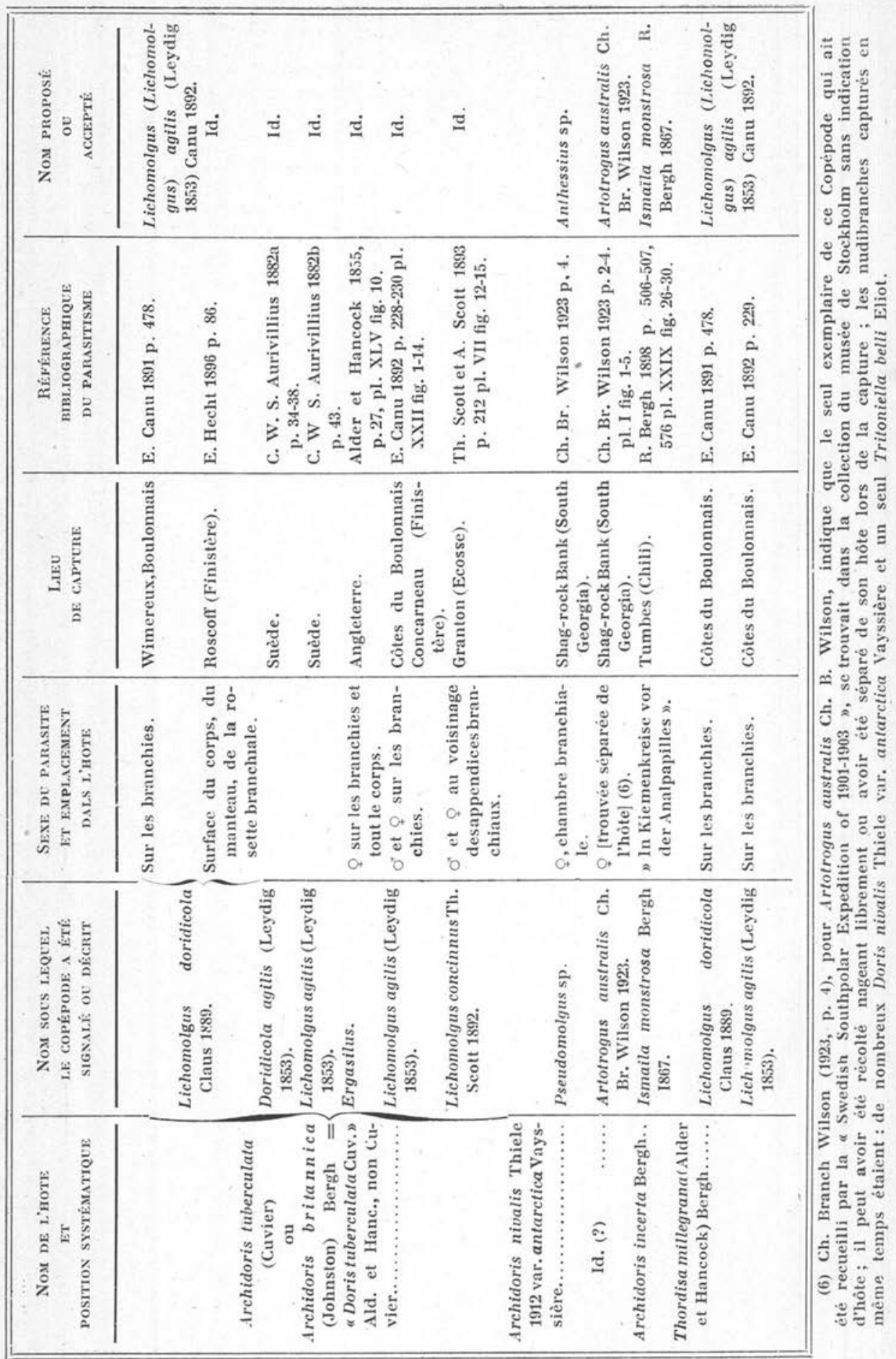




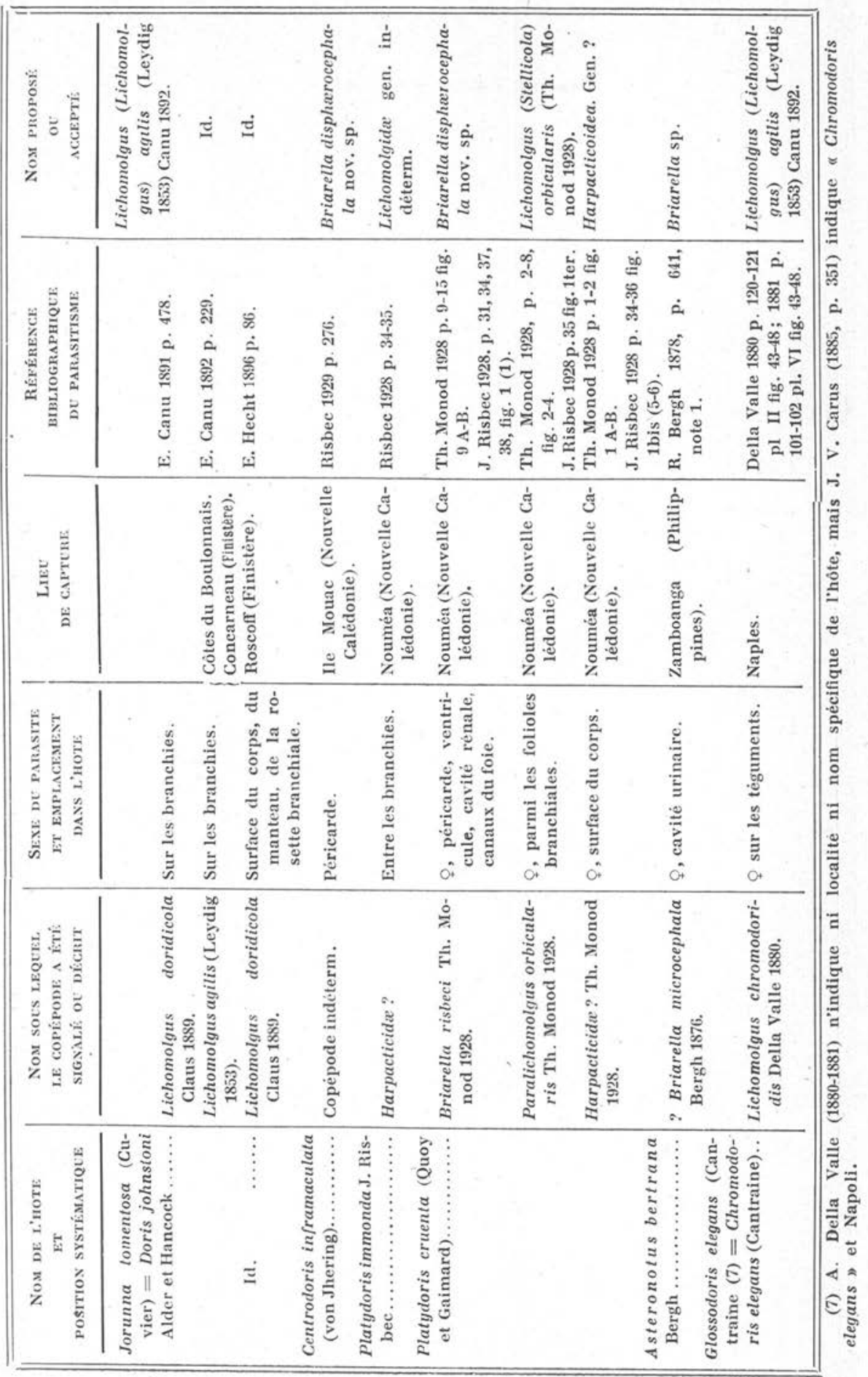




\begin{tabular}{|c|c|c|c|c|c|c|c|}
\hline 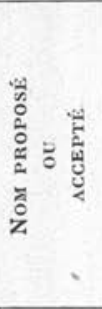 & 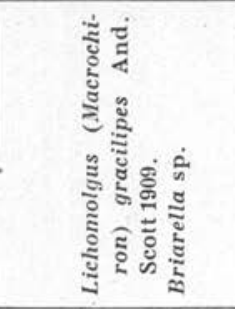 & 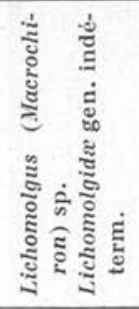 & 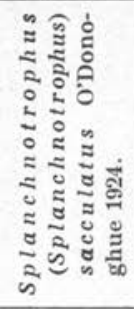 & 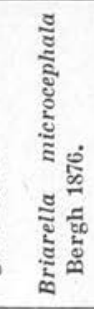 & 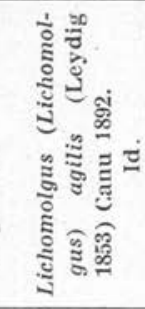 & (2) & $\Xi$ \\
\hline 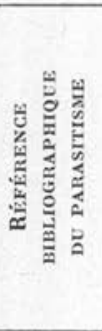 & 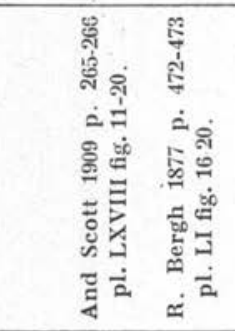 & 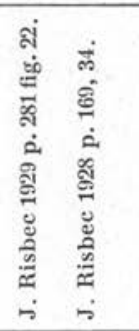 & 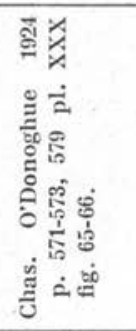 & 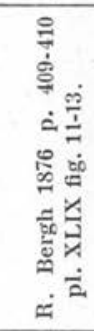 & 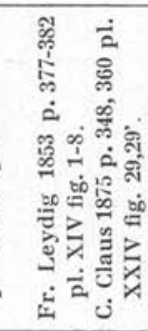 & 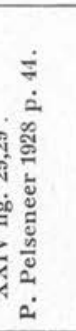 & 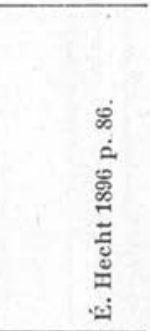 \\
\hline 苞蔍 & 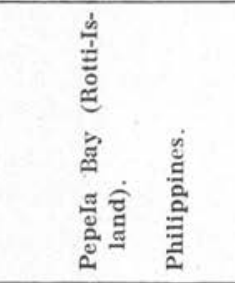 & 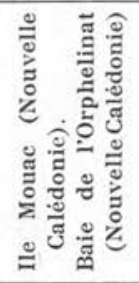 & 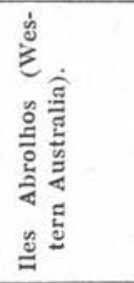 & 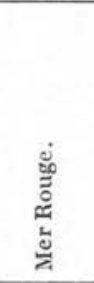 & 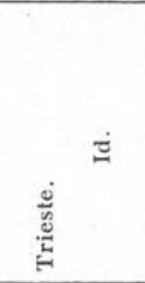 & & 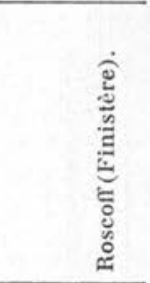 \\
\hline 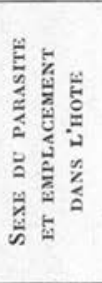 & 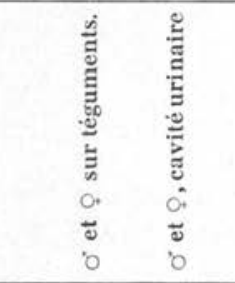 & 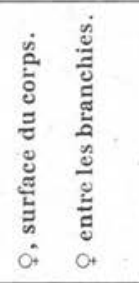 & 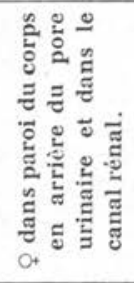 & : & 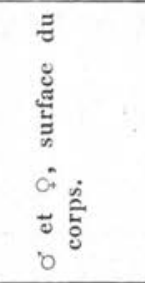 & & 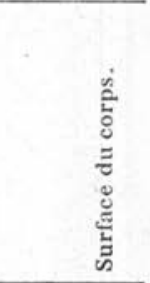 \\
\hline 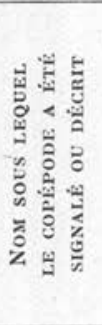 & 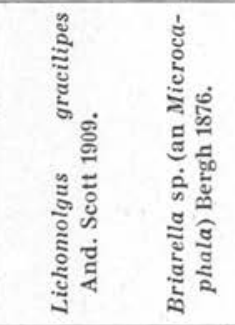 & 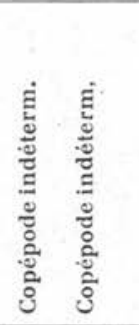 & 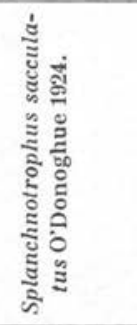 & 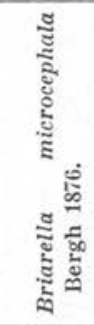 & 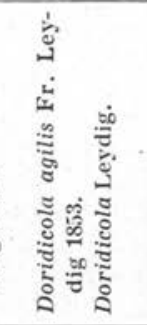 & 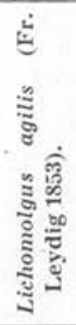 & 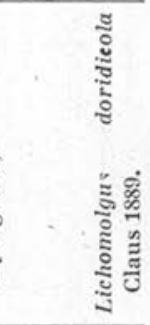 \\
\hline 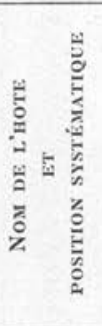 & 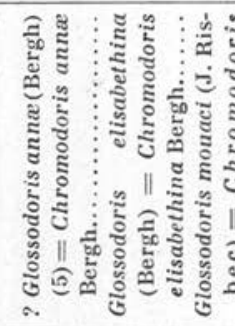 & 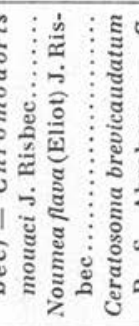 & 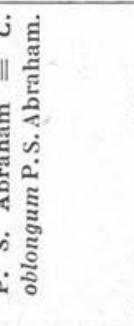 & 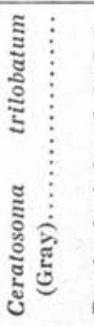 & 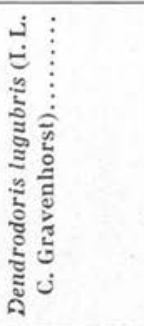 & & 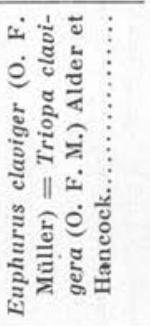 \\
\hline
\end{tabular}




\begin{tabular}{|c|c|c|c|c|c|c|c|c|c|c|c|}
\hline 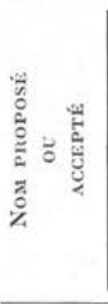 & 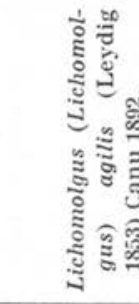 & כ2 & & 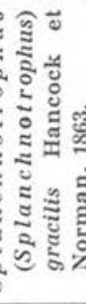 & & ذ் & $\Xi$ & 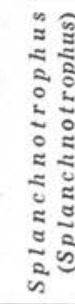 & : & & 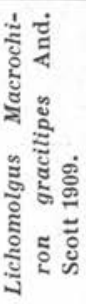 \\
\hline 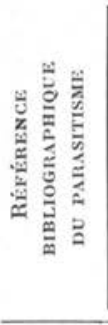 & 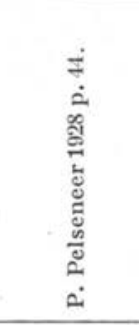 & 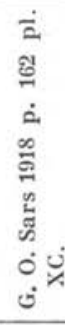 & 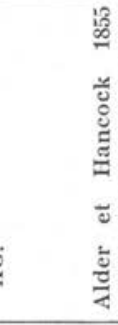 & 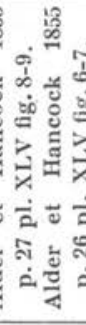 & 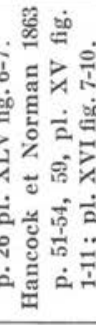 & 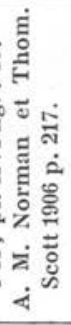 & 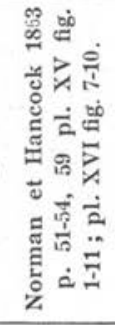 & 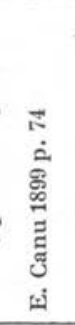 & 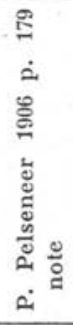 & & 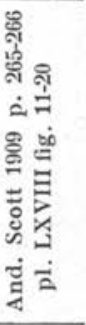 \\
\hline 兽 & & 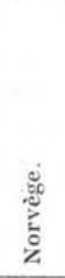 & 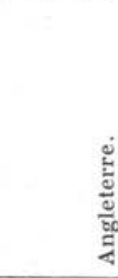 & 惫 & 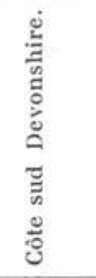 & $\Xi$ & 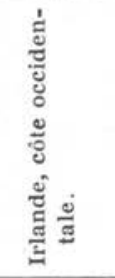 & & & & 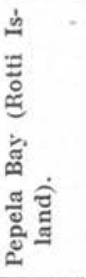 \\
\hline 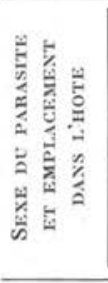 & & $\begin{array}{l}0 \\
0 \\
0\end{array}$ & 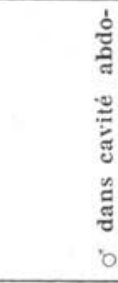 & 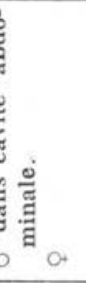 & 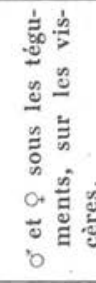 & 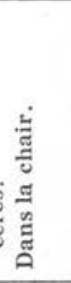 & 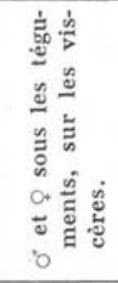 & 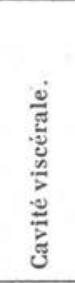 & & & 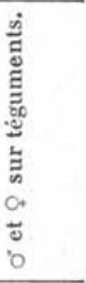 \\
\hline 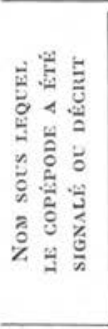 & 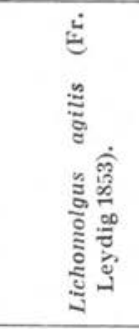 & 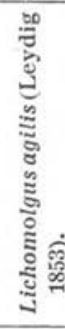 & 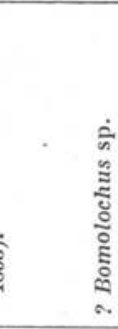 & 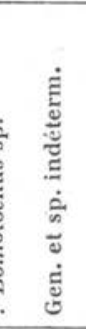 & 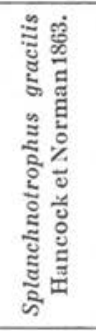 & $\Xi$ & 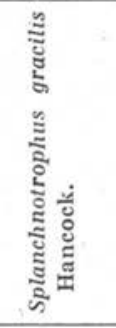 & 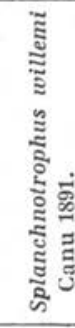 & & & 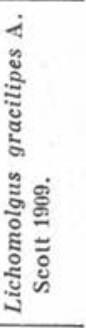 \\
\hline 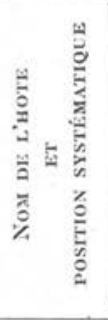 & 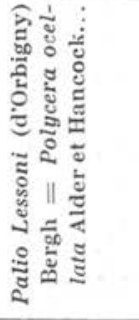 & 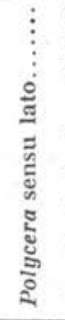 & 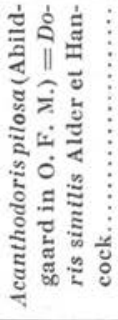 & 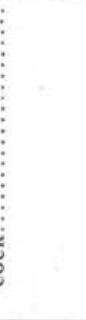 & & & 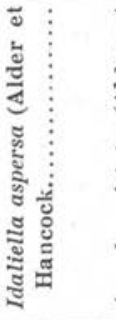 & 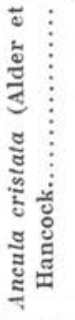 & $\begin{array}{l}\vdots \\
\vdots \\
\vdots \\
\vdots \\
\vdots\end{array}$ & 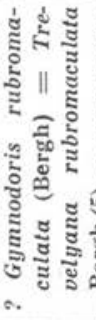 & लै \\
\hline
\end{tabular}




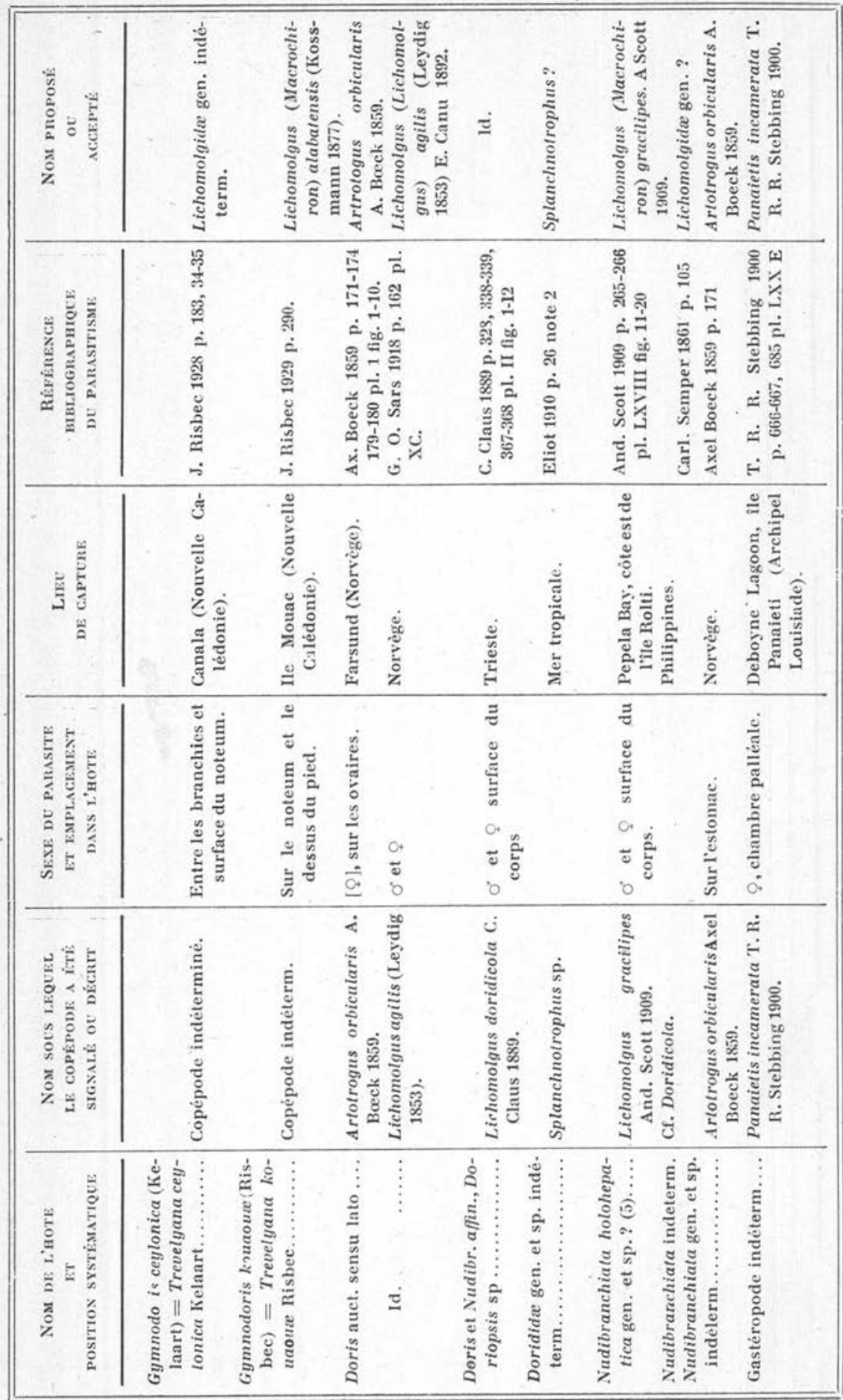




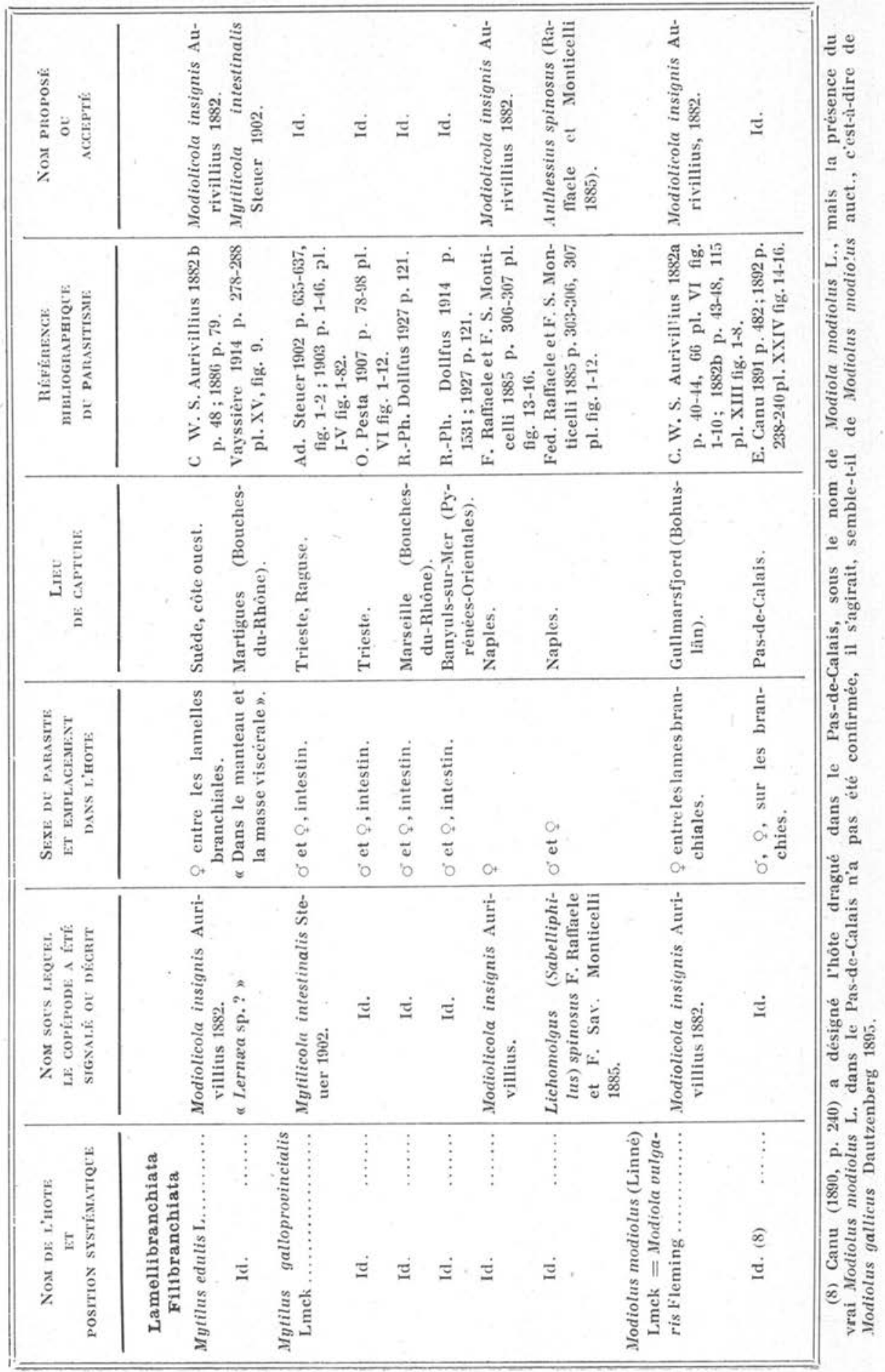




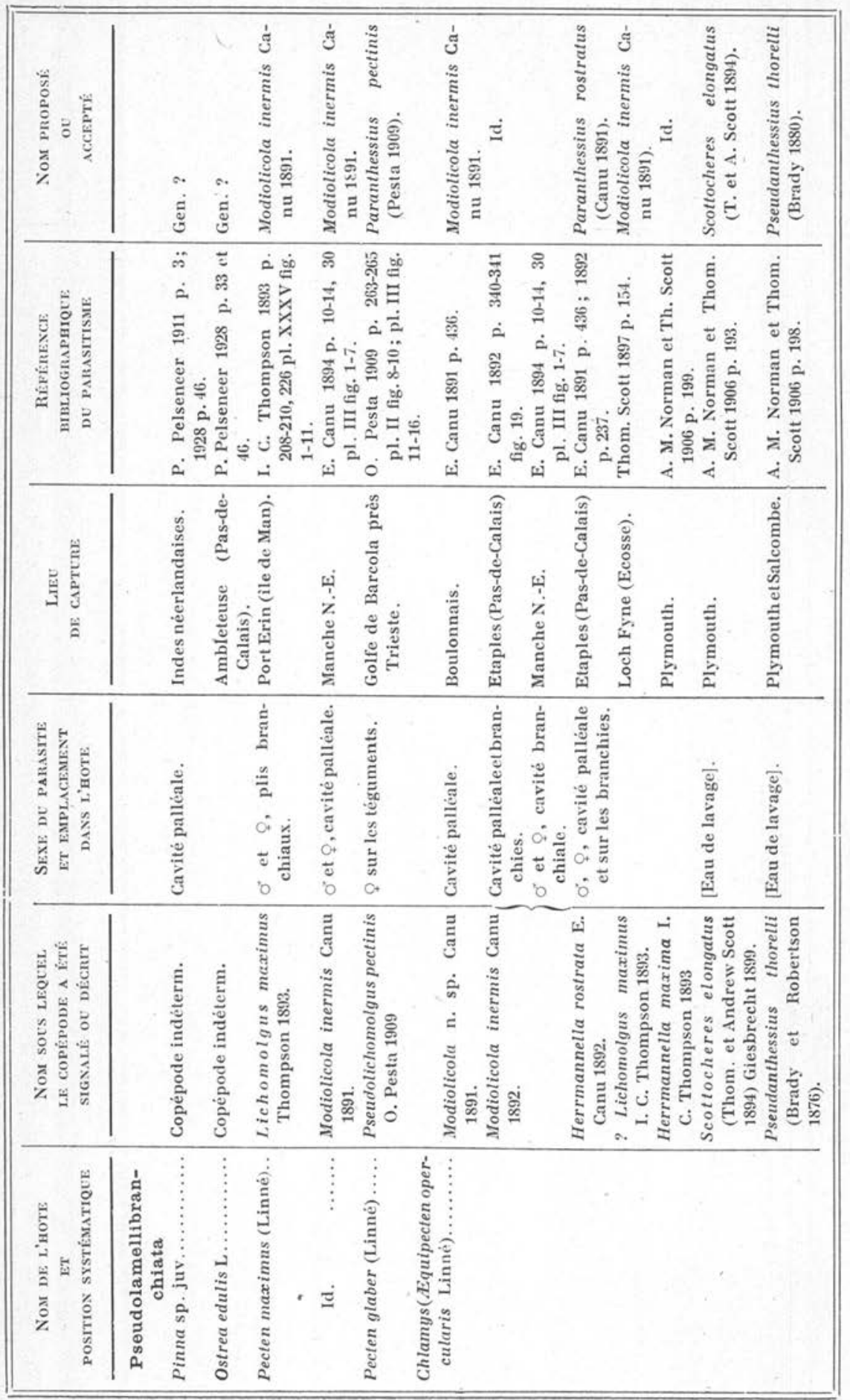




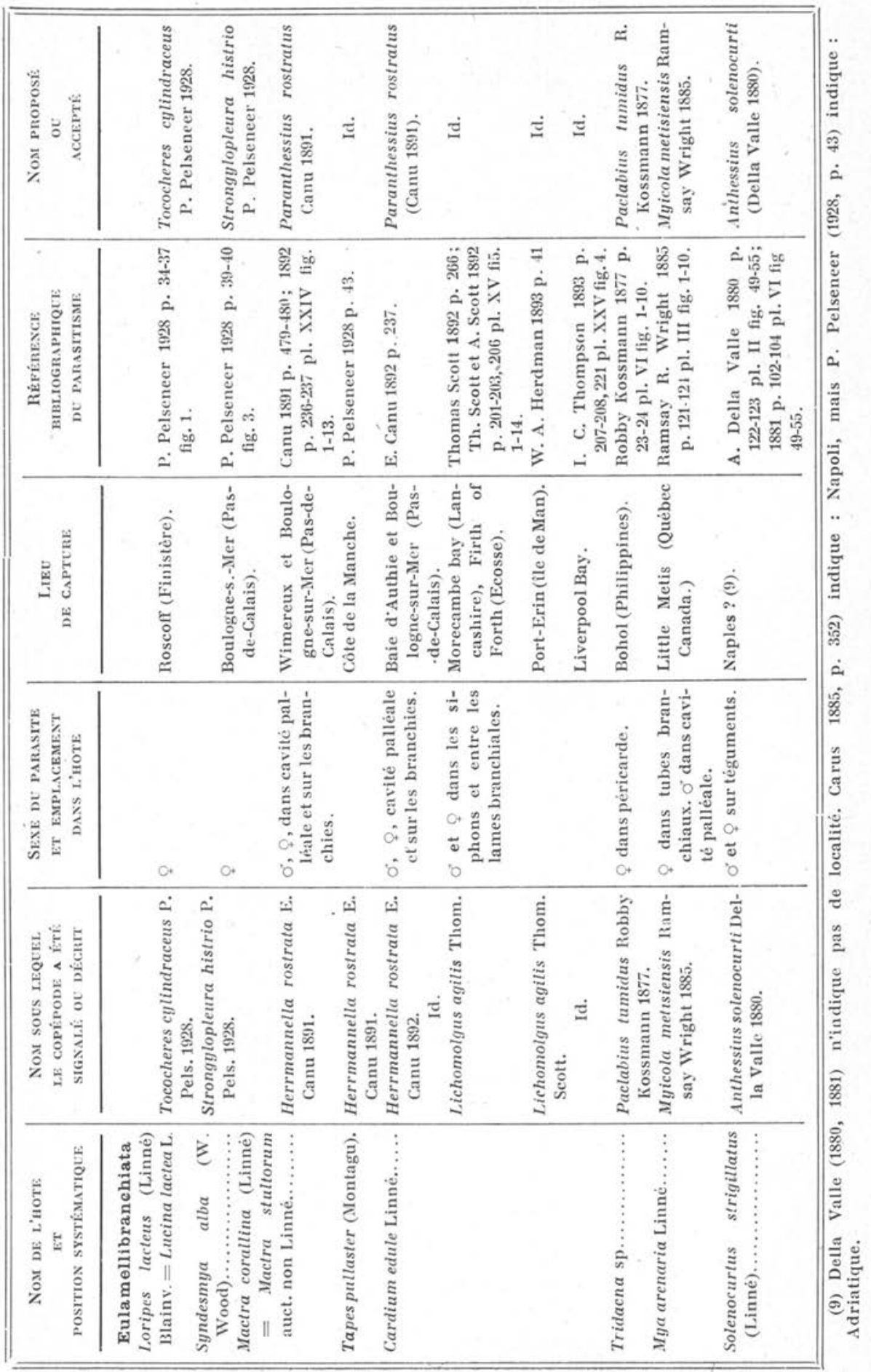




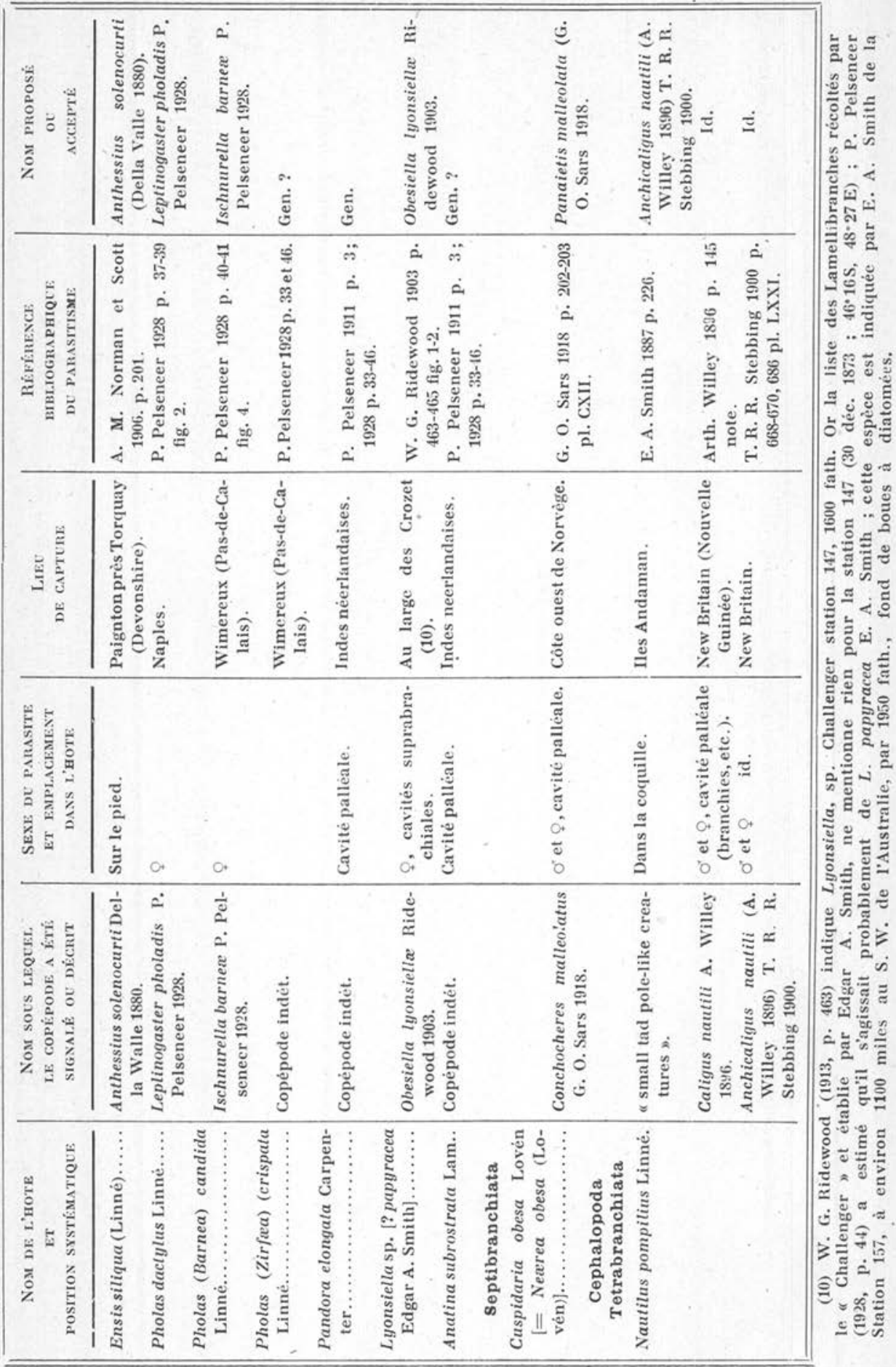




\begin{tabular}{|c|c|c|c|c|c|c|c|c|c|c|}
\hline 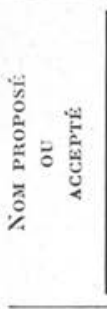 & 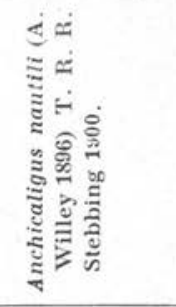 & 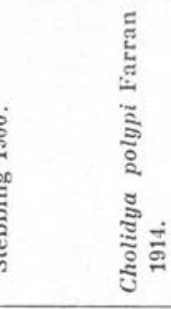 & 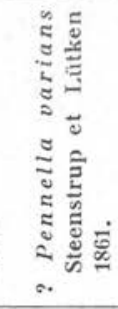 & $\Xi$ & 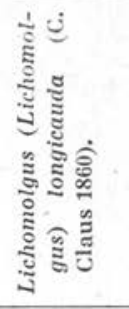 & $\Xi$ & $\Xi$ & $\Xi$ & 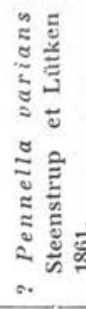 & 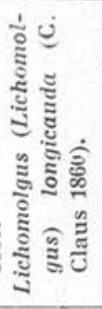 \\
\hline 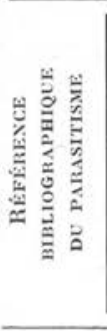 & 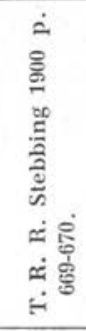 & 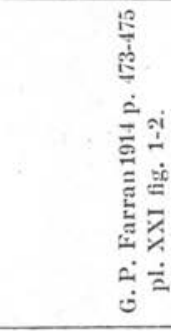 & 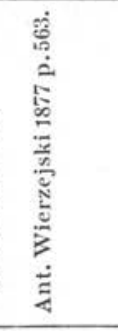 & 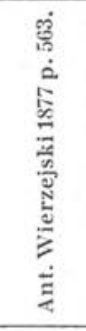 & 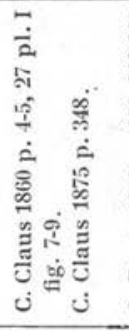 & 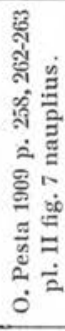 & 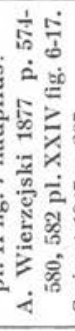 & 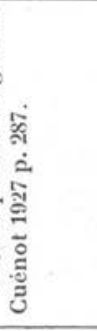 & 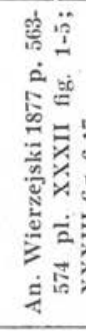 & 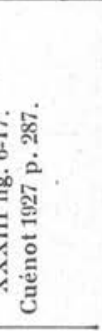 \\
\hline 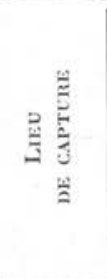 & $\stackrel{\Xi}{\Xi}$ & 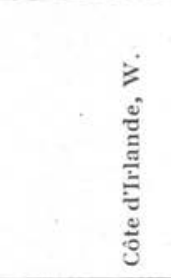 & . $\frac{\frac{9}{u}}{\frac{u}{u}}$ & : & 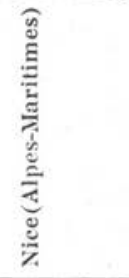 & 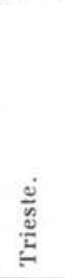 & 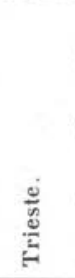 & . & 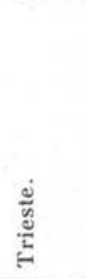 & 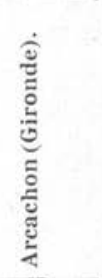 \\
\hline 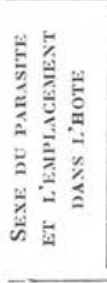 & 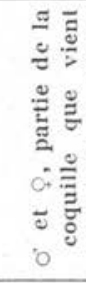 & 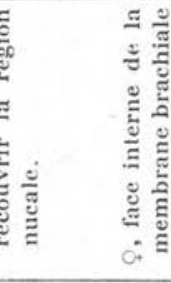 & 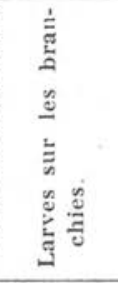 & 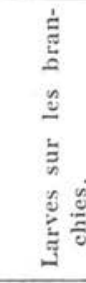 & 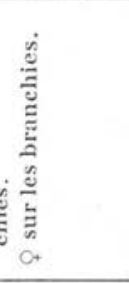 & 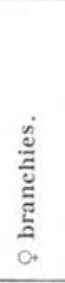 & $\stackrel{\dot{0}}{\tilde{\Xi}}$ & 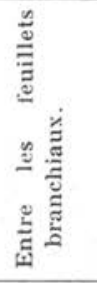 & 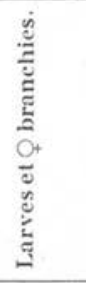 & 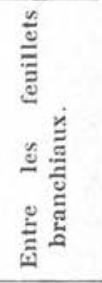 \\
\hline 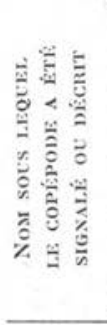 & 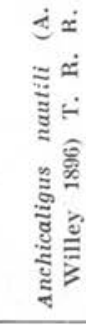 & 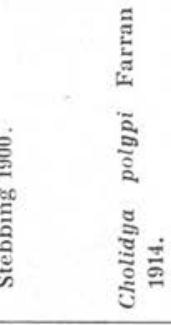 & 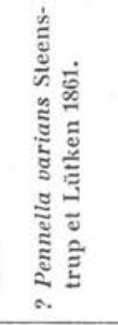 & 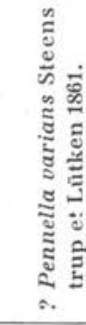 & 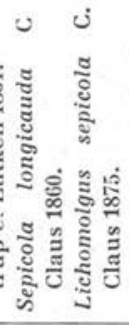 & 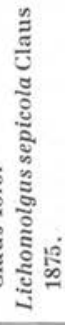 & 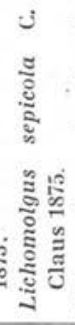 & 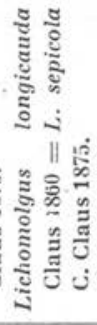 & 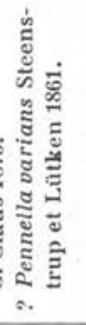 & 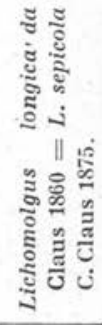 \\
\hline 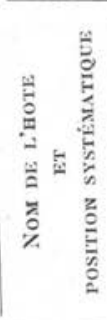 & 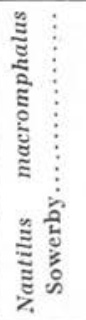 & 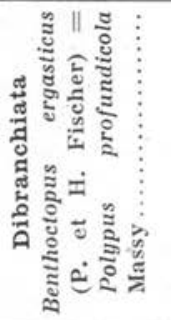 & 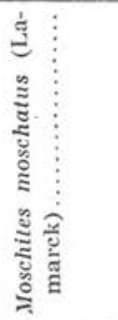 & 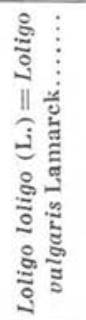 & 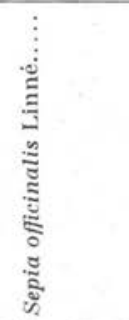 & $\Xi$ & & $\Xi$ & $\Xi$ & 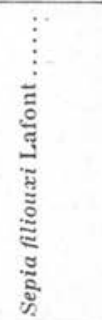 \\
\hline
\end{tabular}




\section{Addendum}

A propos de Lichomolgus agilis (Leydig), nous devons ajouter une localité nouvelle pour la faune française : Mme A. Pruvost-Fol vient de nous envoyer de nombreux spécimens de cette espèce, qu'elle a récoltés sur Archidoris tuberculata (Cuvier), à Banuyls-sur-Mer (PyrénéesOrientales).

A propos de Splanchnotrophus (Splanchnotrophus) sp ? chez Eolidina [=Spurilla] neapolitana Delle Chiaje 1830, nous reproduisons (un peu réduite), une figure publiée par Delle Chiaje (1830, pl. LXXXVIII, fig. 12,$15 ; 1841$, pl. LXXIII, fig. 12, 15), montrant un grand nombre de femelles de Splanchnotrophus in situ chez ce Nudibranche et un individu isolé. Il est évident que le dessinateur a interprété, avec un peu de fantaisie, la morphologie des parasites, a exagéré leur nombre et schématisé avec beaucoup d'imagination leurs emplacements dans l'hôte. Nous n'avons pu trouver où Delle Chiaje, dans son texte, a fait mention de ces parasites; la légende de la planche ne fournit aucune explication (1).

(1) Cette figure a été aimablement signalée à notre attention par Madame A. Pruvot-Fol.

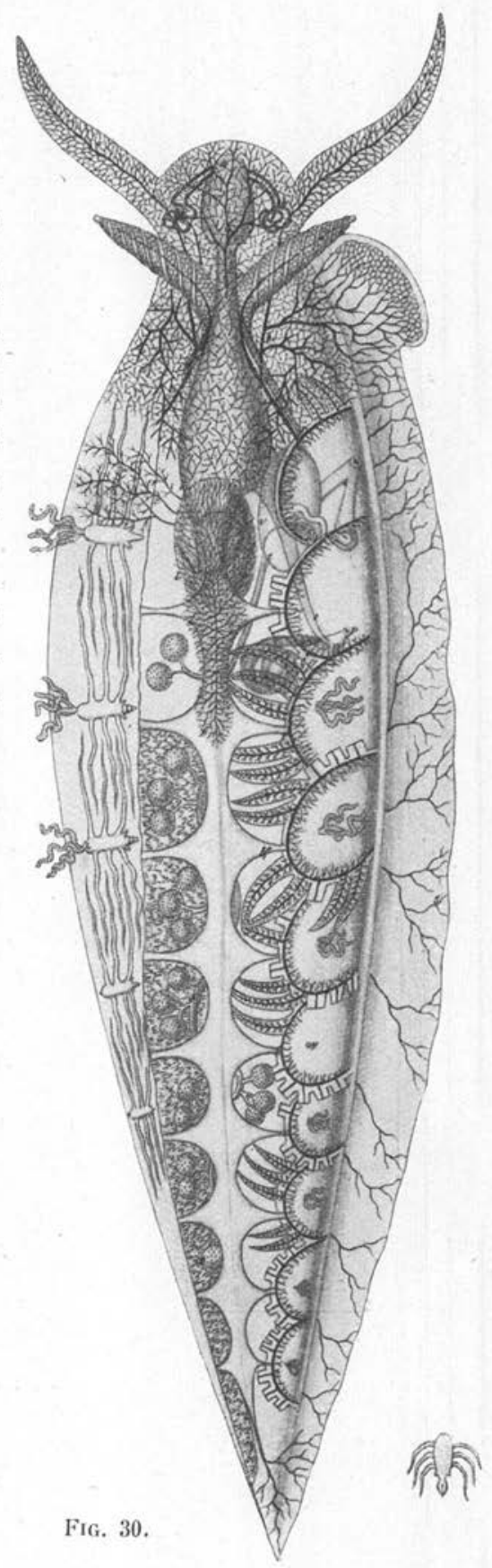




\section{BiBLIOGRAPHIE}

Alder (J.) et Hancock (A.). - A monograph of British Nudibranchiate Mollusca. Ray Society. London, Part VII, 1855, 2 p. n. num. + p. 1-54 + Appendix I-XL +4 p. n. num., pl. XXIa, XXVII, I, II, XXXVIIIa, XLV suppl., XLVI suppl., XLVII suppl., XLVIII suppl.

Auniviltius ( C. W. S.). - Bidrag till Kännedomen om Krustaceer, som lefvahos Mollusker och Tunikater. Kongl. Vetenskaps-Akademiens Förhandlingar. Stockholm, 1882, $n^{\circ} 3$, p. 31-67, pl. V-VI ; n० 8, p. 41-117, pl. XIII-XVI.

- Bemerkungen zu einem Aufsatze : « Descripzione di un nuovo Lichomolgus parassita del Mytilus galloprovincialis LK. ", Memoria dei Signori F. Raffaele e F. S. Monticelli (Reale Accademia dei Lincei). Roma 1885. Zoologischer Anzeiger, IX, no 215, 8 Febr. 1886, p. 77-79.

Bassett-Sмiтh (P. W.). - A Systematic description of Parasitic Copepoda found on Fishes, with an enumeration of the known species. Proc. Zoolog. Soc. London, $1899, \mathrm{n}^{\circ} \mathrm{XXIX}$, p. 438-507, pl. XXVI.

- On new parasitic Copepoda from Zanzibar and East Africa collected by Cyril Crossland. Proc. Zoolog. Soc. London, 1903, vol. I, n VII, p. 104-109, fig. 11-12.

Bergh (R.). - Phidiana lynceus og Ismaila monstrosa. Videuskab. Meddel. fra den naturhist. For. $i$ Kjöbenhavn (1866), no 7-9, p. 97-130, pl. III-IV, 1867.

- On Phidiana lynceus and Ismaila monstrosa. Ann. Mag. Nat. History, London (4 s.), $\mathrm{II}, \mathrm{n}^{\circ} 8$, august 1868, p. 133-138, pl. I, fig. 1-22.

Beiträge zur Kenntniss der Aeolidiaden. Verhandl. der Kaiserl. Königl. Zool. botan. Gesellschaft in Wien, XXXIII, 1873, p. 597-628, fig. VII-X.

Malacologische Untersuchungen, II. Hälfte, X Heft (4 Mai 1876), in : Carl Semper. Reise im Archipel der Philippinen, II, p. 377-427, pl. XLIX-LIII.

Id. II. Hälfte, XI. Heft (28 April 1877), in : Carl Semper. Reise im Archipel der Philippinen, II, p. 429-494, pl. LIV-LVII.

Id. II. Hälfte, XIV. Heft (28 Dez. 1878), in : Carl'Semper. Reise im Archipel der Philippinen, II, p. 603-645, I-L, pl. LXVI-LXVIII.

Beiträge zur Kenntniss der Aeolidiaden Pt. VI. Verhandl. der Kaiserl. Königl. zoolog. botan. Gesellschaft in Wien, XXVIII (1878), 1879 p. 553-584, pl. VI-VIII.

Die Opisthobranchier der Sammlung Plate. Zoolog. Jahrbücher. Supplem. IV. Heft 3, 1898 [Fauna chilensis I Bd.], p. 481-582, pl. XXVIII-XXXIII.

Boss (J. E. V.). - Spolia atlanlica. Bidrag til Pteropodernes Morfologi og Systematik samt til kundskaben om deres geografiske udbredelse. Det kongel. Danske Videnskab. Selskabs Skrifter (6), IV, 1886, p. 1-231, pl. I-VIII.

Вовск (А.). - Tvende nye parasitiske krebsdyr, Artotrogus orbicularis og Asterocheres Liljeborgi. Forhandling. Videnskabs-Selskabet $i$ Christiania, 1859, p. $170-181,2 \mathrm{pl}$.

Bonsier (J.). - Contribution à l'étude des Epicarides. Les Bopyrida. Travaux Station Zoolog. de Wimereux, VIII, 20 nov. 1900, p. 1-476, fig. 1-62, pl. I-XLI.

Brady (G. S.). - Contributions to the study of the Entomostraca. VII. A list of the non-parasitic marine Copepoda of the North-east coast of England. Ann. Mag. Nat. Hist., London (4), X, no 55, July 1872, p. 1-17, pl. II-VI.

- A Monograph of the free and semi-parasitic Copepoda of the british Islands, Ray Society, London, III, p. 1-83, pl. LXXXIII-XCIII. 
Brady (G. S.) et Robentson (D.). - Report on Dredging off the coast of Durham and north Yorkshire in 1874. Report of the 45th meeting of the British Assoc. for Adv. Science, held at Bristol in August 1875, p. 185-199 [1876].

Cane (E.). - Les Copépodes marins du Boulonnais. V. Les semi-parasites. Bulletin scientifique France et Belgique, XXIII; (4), II, 1891, p. 467-487.

- Sur quelques Copépodes parasites obserrés dans le Boulonnais. C. R. Acad, Sc., Paris, CXIII, séance du 5-10-1891, p. 435-437.

- Les Copépodes du Boulonnais. Travaux Laboratoire Zool. mar. Wimerenx, VI, 1892 , p. 1-354, fig. 1-20, pl. I-XXX.

- Notes de biologie marine, faunistiques ou éthiologiques. V. Observations sur quelques copépodes parasites des Mollusques comestibles de la Manche. Annales Stalion Aquicole Boulogne-sur-Mer, II, partie 1, janv. 1894, p. 1-28, pl. I-IV.

- Sur Lichomolgus trochi, nov. sp., Copépode nouveau, parasite d'un Mollusque. Travaux de la Station zoologique de Wimereux, VII, (Miscellanées biologiques dédiées au Professenr Alfred Giard). p. 73-79, pl, VIII.

Carus (J. V.). - Prodromus Fauna mediterranea sive descriptio animalium maris mediterranei incolarum..... I, Stuttgart, 1884, p. I-XIII +1-524 + 1 p. Addenda.

C.ıpaṅ̀̀E (R. E.). - Beobachtungen über Analomie und Entwickelungsgcschichle wirbelloser Thiere an der Küsle von Normandie angestellt von Dr. A. René Edouard Claparède..... Leipzig, 1863 , VIII + 120 pages, pl. I-XVIII.

Claus (C.). - Beilräge zur Kenntnis der Entomostraken. 1tes Heft, 1860, Marburg, p. 1-28, pl. I-IV.

- Die freilebenden Copepoden, mil besonderer Berücksichtignng der Fauna Deutschlands, der Nordsee und des Mittelmceres. Leipzig, 1863, X +230 pages, pl. I-XXXVII.

- Neue Beiträge zur Kenntniss parasitischzn Copepoden, nebst Bemerkungen über das System derselben. Zeitschrift für wiss. zool., XXV, 4. H., 25 Juli 1875 , p. $327-360$, pl. XXII-XXIV, fig. 1-41.

- Ueber neue oder wenig bekannte halbparasitische Copepoden, insbesondere die Lichomolgiden und Ascomyzontiden-Gruppe. Arbeiten d. zoolog. Institut Wien, VIII, IIeft 3,1889 , p. 327-370, pl. I-VII.

Cúxot (L.). - Contribution à la faune du bassin d'Arcachon. III. Doridiens. Socièté Scientifique d'Arcachon. Station biologique. Travanx des laboratoires, VII, 1903, p. 1-22, planche, fig. 1-6.

- Contributions à la faune du bassin d'Arcachon. IX. Revue générale de la faune et bibliographie. Bull. Stalion biologique d'Arcachon, XXIV, 1927, p. 229-308, fig. 1-5.

Dana (J. D.). - Crustacea. Part II. United States Exploring Expeditic $n$ during the years 1838, 1839, 1840,1841, 1842. XIV, Philadelphia, 1853, p. 689-1618, 1 carte. Atlas, 27 pages, 96 planches, 1855 .

Delda VALLE (A.). - Sui Coriceidi parassiti e sull' anatomia dell' gen. Lichomolgus. Memor. della classe di Scienze fis. mat. e nat. della Reale Accad. Lincei. Roma, Ser. 3a, V, 1880, p. 107-124, pl. I-II, fig. 1-58.

- Sui Coriceidi parassiti e sull' anatomia del gen. Lichomolgus. Mitt. Zoolog. Station Neapel, II, 1 Heft, 1881, p. 83-106, pl. V-VI, fig. 1-58.

Delde Chiase (S.). - Memorie sulla storia e notomia degli. - Animali seuza verlebre del Regno di Napoli ; V, 1830, pl. LXX-CIX.

- Descrizione e Notomia degli Animali invertebrati della Sicilia citeriore Animali senza vertebre del Regno di Napoli, VI, 1841, pl. L-LXXXNI. 
Doltrus (R.-Ph.). - Trochicola enterica, nov. gen., nov. sp., eucopépode parasite de l'intestin des troques. C. R. Acad. sc., Paris, CLVIII, 25 mai 1914, p. 1528-1531, fig. 1-2.

- Notules sur des copépodes parasites de la faune française. I-III. Bull. soc. zool. France, LII, no 2, 30-4-1927, p. 119-121.

ELIOT (C.). - A monograph of the British Nudibranchiate Mollusca. Part VIII (Supplementary). The Ray Society for the year 1909, p. 1-198, pl. I-VIII, 1910.

FarRaN (G. P.). - Description of a Harpacticid Copepod parasitic on an Octopus. Ann Mag. Nat. Hist., London (8). XIII, May, 1914, n 17, p. 472-475, pl. $\mathrm{XXl}$, fig. $1-12$.

Ganstang (W.). - A complete list of the Opisthobranchiate Mollusca found at Plymouth. Journal Marine biolog. As:oc., I (New Series), n* 4, Nov. 1890, p. $399 \cdot 457, \mathrm{pl}$. XXVII-XXVIII.

Genstaecker (A.). - Crustacea (Erste Hälfte). Die Klassen und Ordnungen der Arthropoden. Bronn's Thier-Reich, V, 1 Abth., 1866-1879, p. 1-1320, pl. I-XLIX.

Gnssrecht (W.). - System der Ascomyzontiden, einer semiparasitischen Copepoden-Familie. Zoolog. Anzeiger, XX, n०521, 11 janv. 1897, p. 9-14; $\mathrm{n}^{\circ} 522,25$ janv. 1897 , p. 17-24.

- Die Asterocheriden des Golfes von Neapel. Fauna und Flora des Golfes von Neapel, XXV. Monographie, 1899 , VI + 217 p., pl. I-XI.

Hancock 'A.) et Noryan (A. M.). - On Splanchnotrophus, an undescribed Genus of crustacea, parasitic in Nudibranchiate Mollusca. Trans. Linnean Soc. London, XXIV, part 2, 1863, p. 49-60, pl. XV-XVI.

Hвснт (E.). - Sur un nouveau Copépode parasite de Nudibranches. Archives zool. expér. (3), I, 1893, Notes et Revue, p. XIII-XVI, fig. 1.

- Contribution à l'étude des Nudibranches. Mém. Soc. Zool. France, VIII, 1895, $n^{\circ} 4$, p. 539--711 [p. 1-175], pl. I-V, fig. 1-82, 1896.

Herdman (W. A.). - Sixth Annual Report of the Liverpool Marine Biology Committee, and their Biological Station at Port Erin, p. 3-54, fig. texte 1-18, pl. I-VI [from Trans. Biolog. Soc. Liverpool, VIII].

- Eight Annual Report of the Liverpool Marine Biology Committee and their Biological Station at Port Erin. Proc. and Trars. Liverpool Biolog. Soc., IX, 1895, p. $26 \cdot 74,1$ fig. texte, pl. I-II.

Kossmann (R.). - Enlomostraca; in : R. lossmann, Zoologische Ergebnisse einer.... Reise in die Küstengebiete des Rothen Meeres, Erste Hälfte, IV, 1877 , p. 1-24, pl. I-VI.

LEYDiG (F.). - Neuer Schmarotzerkrebs auf einem Weichthier. (Zoologische Notizen. 1). Zeitschrift für wiss. Zool., IV, 18-4, 3-4 Heft, 1853, p. 377-387, pl. XIV, fig. 1-8.

MoNon (Th.). - Sur quelques copépodes parasites de Nudibranches. Bull. Institut océanogr. Monaco, no 509, 25 janv. 1928, p. 1-18, fig. 1-12.

MüLler (O. F.). - Entomostraca sen Insecta testacea, qux in aquis Danix et Norvegia reperit, descripsit et iconibus illustravit... Lipsiae et Havniae, 1785 , p. 1-134, pl. 1-XXI.

Norman (A. M.) et ScotT (Th ). - The Crustacea of Devon and Cornwall. London, 1906, p. I-XV + 1-232, pl. I-XXIV.

OAкr.ey (C. L.). - The Chondrucanthida (Crustacea : Copepoda) with a description of five new genera and one new species. Parasitology, XXII, n², 24 March 1930 , p. $182-201$, fig. $1-8$. 
OdHNer (Nils Hi.). - Norwegian Opisthobranchiate Mollusea in the collections of the zoological Museum of Kristiania. Meddelelser fra det zoologiske Mus. Kristiania. Nr. 1. (Nyt Magazin for Naturvidenskaberne B. 60.), 1922, p. 1-47, fig. 1-15.

O'Donoghue (Chas. H.). - Report on Opisthobranchiata from the Abrolhos Islands, Western Australia, with Description of a new parasitic Copepod. Journ. of the Linn. Soc. London, XXXV, n ${ }^{\circ}$ 237, zoology, Febr. 14, 1924, p. 521-579, pl. XXVII-XXX, fig. 1-66.

Öada (Yô K.). - Stanrosoma, copépode parasite d'une actinie: description de Staurosoma caulleryi sp. n. Annotationes zool. japon, $\mathrm{XI}, \mathrm{n}^{\circ} 2,1927$, p. 173-182, 1 fig. texte, 1 pl., fig. 1-4.

OKEN (L.). - Lehrbuch der Nalurgeschichte. Dritter Theil, Zoologie, Iena. Vol. I, 1815 , XXVIII $+850+$ XVIII p. ; Vol. II, 1816 , p. I-XVI $+1-650+$ 651-1270. Atlas p. I-IV, pl. I-XL.

Peanson (J.). - A List of the marine Copepoda of Ireland. Part I. Littoral forms and fish parasites. Fisheries, Ireland, Sci. Invest., 1904 III, (nov. 1905), p. $143-170$ [p. 1-30].

Pelseneer (P.). - Recherches sur divers Opisthobranches. Mém. couronnés et Mém. savants étrangers publiés par l'Acad. Royale des Sc. de Belgique, 4. LIII, 1894, p. I-III + 1-157, pl. I-XXV, fig. 1-227.

- Amphineures, Gastropodes et Lamellibranches. Résultats Voyage S. Y. Belgica, 1903, p. 1-85, pl. I-IX.

- Trématodes parasites de Mollusques. Bull. scient. France et Belgique, XL, (5 s., t. IX), p. 161-186, pl. VIII-XII.

- Les Lamellibranches de l'Expéciition du Siboga. Partie anatomique. SibogaExpeditie. Monogr. LIIIa, 1911, p. 1-125, pl. I-XXVI.

- Ethologie de quelques Odostomia et d'un Monstrillide parasite de l'un d'eux. Bull. Scient. France et Belgique (7), XLVIII, p. 1-14, pl. I-III.

- Les Parasites des Mollusques et les Mollusques parasites. Bull, Soc. zool. France, LIII, n 3 , 10 mai 1928 , p. 158-189.

- Copépodes parasites de Mollusques. Annales Soc. Royjale zoologique de Belgique, LiX, 1928, p. 33-49, fig. 1-5.

Pesta (O.). - Die Metamorphose von Mytilicola intestinalis Steuer. Zeitsch, für wiss. Zool., LXXXVIII, 1 Heft, 1 Okt. 1907, p. 78-98, pl. VI, fig. 1-12.

- Beiträge zur Kenntnis parasitischer Copepoden. Denkschr. K. Akad. Wissensch. Wien. Math. Naturwiss. Klasse, LXXXIV, 1909, p. 257-267, pl. I-III, fig. 1-16.

Raffaele (F.) et Monticella (F. S.). - Descrizione di un nuovo Lichomolgus parassita del Mytilus galloprovincialis Lk. Memorie della classe di Sc. fisiche, matem. e naturali. Atti $R$. Accad. dei Lincei., Roma (4), I, 1885, p. 302-307, 1 pl., fig. 1-16.

Ridewood (W. G.). - On Obesiella lyonsiellae, a new genus of copepod crustacean. Journ. Linn. Soc. London, Zoology, XXVIII, May 1, 1903, p. 463-465, 2 fig.

Rıssec (J.). - Contribution à l'étude des Nudibranches néo-calédoniens. Faune des Colonies Françaises, II, 1928, p. 1-328, fig. texte 1-98+1 bis +1 ter ; pl. A-D, I-XII, 3 cartes.

- Nouvelle contribution à l'étude des Nudibranches néo-calédoniens. Annales Inst. océanogr. Paris, VII, 1929, fase. 7 p. 263-298, fig. texte 1-69, pl. I, fig. 1-6.

- Observations biologiques sur quelques Mollusques de la Nouvelle-Calédonie. Bull. Muséum Paris, N. S., II, nov. 1930, no 6, p. 660-664, fig. 1-8.

SARs (G. O.). - An account of the Crustacea of Norway : IV, Copepoda Calanoida, part I et II, p. 1-28, pl. I-XVl, 1901. 
Sars (G. O.). - Liste systématique des Cyclopoidés, Harpacticoidés et Monstrilloidés recueillis pendant les campagnes de S. A. S. le Prince Albert de Monaco, avec descriptions et figures des espèces nouvelles. Bull. Instilul Océanogr. Monaco, $\mathrm{n}^{\circ} 323,30$ sept. 1916, p. 1-15, fig. 1-8.

- An Account of the Crustacea of Norway, VI, Copepoda Cyclopoida, Bergen, 1918, XIII + 225 p., pl. I-CXVIII.

Sans (M.). - Beskrivelse med Afbildninger af fire nye parasitiske Copepoder. Forhandlingar $i$ Vidensk. Selskabet $i$ Christiania, aar 1861, p. 134-141, 1862.

Scotr (A.). - The Copepoda of the Siboga Expedition. Part 1. Free swimming, littoral and semi-parasitic Copepoda. Siboga-Expeditie, XXIX a. Livraison 44, Sept. 1909, p. 1-323, pl. I-LXIX.

Scotr (Th.). - Additions to the Fauna of the Firth of Forth. Part IV, Tenth Annual Report to the Fishery Board for Scotland for 1891, part III, Scientif. Investig. p. 244-272, pl. VII-XIII, 1892.

- The marine Fishes and invertebrates of Loch Fyne. Fiveteenth Annual Report to the Fishery Board for Scolland for 1896, part III, Scientif. Investig. p. 107-174, pl. I-III, 1897.

Scotr (Th. et A.). - On some new or rare Crustacea from the Firth of Forth. Ann. Mag. Nat. History (6), X, n 57, 1892, p. 201-206, pl, XV-XVI.

On some new or rare scottish Entomostraca. Ann. Mag. Nat. Hist. (6), XI, $\mathrm{n}^{\circ}$ 63, March 1893, p. 210-215, pl. VII fig. 1-15, pl. VIII fig. 1-14.

On some new and rare Crustacea from Scotland. Ann. Mag. Nat. Hisl. (6), XIII, n ${ }^{\circ} 74$, Febr. 1894, p. 137-149, pl. VIII-IX.

On some new and rare British Copepoda. Ann. Mag. Nat. Hist. (6), XVI, no 95 , Nov. 1895, p. 353-362, pl. XV-XVII.

SEMPln (C.). - Reisebericht des Herrn Dr. Semper (Fortsetzung). Zeilschrift für wissensch. Zoologie, XI, 1 Heft, 1 Febr. 1861, p. 100-108.

Sмrтн (E.). - Note on the pearly Nautilus. Journal of conchology, V, Oct. 1887, p. 226-227.

Strbming (T. R. R.). - On Crustacea brought by Dr. Willey from the South Seas. A. Willey's Zoological Results, Cambridge, V, dêc. 1900, p. 605-690, pl. LXIV-LXXIV.

Steenstrup (J. Jap. Sm.) et Lërken (Cl. Fr.). - Bidrag til Kundskab om det aabne Havs Suyltekrebs og Lerneer samt om nogle andre nye eller bidtil kun ufuldstaendigt kjendte parasitiske Copepoder. Dct Kongl. Danske Vidensk. Selskabs Skrivter (5 Rakke), V Bind, 1861, p. 341-432, pl. I-XV, fig. 1-40.

Steven (A.). - Mytilicola intestinalis n. gen., n. sp., aus dem Darm von Mytilus galloprovincialis Lamck (Vorläufige Mittheilung). Zoolog. Anzeiger, XXV, 1 sept. $1902, \mathrm{n}^{\circ} 680$, p. 635-637, fig. 1-2.

- Mylilicola intestinalis n. gen., n. sp. Arbeilen Zoolog. Inslitul Wien, XV, 1903 , p. 1-46, pl. I-V.

Tchang Sr. - Contribution à l'étude des mollusques opisthobranches de la côte provençale. Thèse Fac. Sc., Lyon, nov. 1931, 221 p., 67 fig., 8 pl., 2 cartes.

Thompson (I. C.). - Revised Report of the Copepoda of Liverpool Bay. Proc. Trans. Liverpool biolog, Soc., VII, 1893, p. 175-230, pl. XV-XXXV.

Thompsin (I. C.) et Scotr (A.). - Report on the Copepoda collected by Professor Herdman, at Ceylon, in 1902. Report of the Govern. of Ceylon on the Pearl Oyster Fisheries of the Gulf of Manaar, Part. I, Supplem. Reports, VII, 1903, p. $227-307, \mathrm{pl}$. I-XX.

Thоnell (T.). - Bidrag till kännedomen om krustaceer, som lefva $\mathrm{i}$ arter af slägtet Ascidia L. Kongl. Svenska Vetenskaps Akad. Handl. Stockholm, Ny Föld III, no 8,1860 , p. 1-84, pl. I-XIV, fig. 1-22. 
VAYssiÈre (A.). - Recherches zoologiques et anatomiques sur les Mollusques opisthobranches du golfe de Marseille. $3^{\circ}$ partie. Nudibranches (suite et fin). Annales Mus. hist. nat. Marseille, VI, Zool. Mém. no 1, 1901, p. 1-130, pl. I-VII.

- Recherches zoologiques et anatomiques sur les Mollusques opisthobranches du golfe de Marseille. Supplément. Annales Mus. hist. nat. Marseille, VIII, section zool. Mém. no 3, 1903, p. 69-108, pl. II-II1, fig. 1-42.

- in Catllol (H.) et Vayssière (A.). Zoogéographie; in "Les Buuches du Rhône. Encyclopédie départementale ), $3^{\circ}$ partie, tome XII, chapitre VIII, 1914, p. 239-380, pl. XII-XXI.

Wıвzелsкi (A.). - Ueber Schmarotzerkrebse von Cephalopoden. Zeitschrift für wiss. Zool., XXIX, 4 Heft, 18 Olk 1877, p. 562-582, pl. XXXII-XXXIV, fig. 1-32.

WiLlex (A.). - Letters from New Guinea on Nautilus and some other Organisms. Quart. Journ. Microscop. Science, XXXIX, n 154, Aug. 1896, p. 145-180, fig. $1 \cdot 24$.

Wilson (Ch. Br.). - New species and a new genus of parasitic Copepoda. Proc. U. S. Nat. Mus., LIX, no 2354,1921 , p. 1-17, pl. I-VII.

- North american parasitic copepods belonging to the family Dichelesthizdre. Proc. U. S. Nat. Mus., LX, art. 5, no 2400, 1922, p. 1-100, pl. I-XIII, fig. 1-105.

- Parasitic Copepods in the collection of the Riksmuseum at Stockholm. Arkiv f. zool., XV, no 3, 1923, p. 1-15, pl. I-II.

Wright (R. R.). - A parasitic Copepod of the Clam. American Naturalist, XIX, Febr. 1885, p. 118-124, pl. III, fig. 1-10.

Zulueta (A.). - Los Copepodos parasitos de los Celentereos. Memorias Reale Soc. Esp. Hist. Nat., VII, 1911, p. 5-58, fig. 1-39.

NOTE AJOUTĖE APRĖS LA CORHECTION DES ÉPREUVES

Depuis l'impression de ce travail, Mme A. Pruvot-Fol nous a remis une préparation de deux $\nmid$ ovigères d'un copépode trouvé par elle, autrefois, vraisemblablement chez un mollusque.

Il s'agit d'une forme très voisine de plusieurs de celles trop sommairement décrites par $P$. Pelseneer et de position systématique incertaine (Lichomolgida ?). Une description complète, avec figures, de ce parasite inédit paraitra dans le $\mathrm{n}^{\circ} 3$ (mai 1932) de ces Annales, sous le nom de Strongylopleura (gen. incert.) pruvoti, nom. provis.

Laboraloire des pèches coloniales du Muséum Nalional d'Hisloire Naturelle de Paris. 